\title{
Substrate-Controlled Selectivity Switch in the Three-Component Coupling Involving Arynes, Aromatic Tertiary Amines, and $\mathrm{CO}_{2}$
}

\author{
Sachin Suresh Bhojgude, ${ }^{\mathrm{a}, \mathrm{b}}$ Tony Roy, ${ }^{\mathrm{a}, \mathrm{b}}$ Rajesh G. Gonnade ${ }^{\mathrm{c}}$ and Akkattu T. \\ $\mathrm{Biju}^{*, \mathrm{a}, \mathrm{b}}$ \\ ${ }^{a}$ Organic Chemistry Division, ${ }^{c}$ Center for Materials Characterization, \\ CSIR-National Chemical Laboratory (CSIR-NCL), \\ Dr. Homi Bhabha Road, Pune - 411008, India \\ ${ }^{b}$ Academy of Scientific and Innovative Research (AcSIR), New Delhi 110020, India \\ at.biju@ncl.res.in
}

\section{Supporting Information}

$\begin{array}{ll}\text { 1. General Information } & \text { S2 }\end{array}$

2. General Procedure for the MCC Involving Arynes, Tertiary Amines and $\mathrm{CO}_{2} \quad \mathrm{~S} 3$

3. General Procedure for the MCC Involving Arynes, Electron-poor Tertiary Amines and $\mathrm{CO}_{2} \mathrm{~S} 3$

4. Mechanistic Experiments $\quad$ S4

5. Synthesis and Characterization of 2-Arylamino Benzoate Derivatives $\quad$ S18

6. Synthesis and Characterization of 2-Aminoaryl Benzoate Derivatives $\quad$ S29

$\begin{array}{ll}\text { 7. Synthesis and Characterization of 10-Methylacridin-9(10H)-one } & \text { S37 }\end{array}$

8. Synthesis and Characterization of 1-Phenyl-1,2-dihydro-4H-benzo[d][1,3]oxazin-4-one S39

9. ${ }^{1} \mathrm{H}$ and ${ }^{13} \mathrm{C}$ NMR Spectra of 2-Arylamino Benzoate and

2-Aminoaryl Benzoate Derivatives $\quad$ S41 


\section{General Information}

Unless otherwise specified, all reactions were carried out under an atmosphere of argon in flame-dried reaction vessels with Teflon screw caps. Reaction temperatures are reported as the temperature of the bath surrounding the reaction vessel. $30{ }^{\circ} \mathrm{C}$ corresponds to the room temperature of the lab, when the experiments were carried out. THF was freshly purified by distillation over Na-benzophenone and was transferred under argon. 18-Crown-6 was recrystallized from dry $\mathrm{CH}_{3} \mathrm{CN}$, and $\mathrm{KF}$ was dried by heating at $110{ }^{\circ} \mathrm{C}$ for $12 \mathrm{~h}$ and left to cool under argon and stored in glove box. The 2(trimethylsilyl)phenyl trifluoromethane sulfonate 1a and the other symmetrical and unsymmetrical aryne precursors $\mathbf{1 b - g}$ were synthesized following

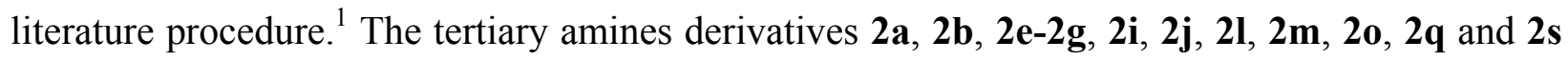
were purchased from either from Sigma Aldrich or Alfa Aesar and used as received, without further purification. Tertiary amine derivatives $\mathbf{2 c}, \mathbf{2 d}, \mathbf{2 h}, \mathbf{2 n}$ and $\mathbf{2 p}$ were synthesized following literature procedure. ${ }^{2}$

Analytical thin layer chromatography was performed on TLC Silica gel $60 \mathrm{~F}_{254}$. Visualization was accomplished with short wave UV light or $\mathrm{KMnO}_{4}$ staining solutions followed by heating. Chromatography was performed on silica gel (230-400 mesh) by standard techniques eluting with solvents as indicated.

All compounds were fully characterized. ${ }^{1} \mathrm{H}$ and ${ }^{13} \mathrm{C}$ NMR spectra were recorded on Bruker AV 400, 500 in solvents as indicated. Chemical shifts $(\delta)$ are given in ppm. The residual solvent signals were used as references and the chemical shifts converted to the TMS scale $\left(\mathrm{CDCl}_{3}: \delta \mathrm{H}=7.26 \mathrm{ppm}, \delta \mathrm{C}=77.16 \mathrm{ppm}\right)$. Infrared spectra were recorded on a Bruker Alpha-E Infrared Spectrophotometer. The wave numbers (n) of recorded IR-signals are quoted in $\mathrm{cm}^{-1}$. Gas Chromatography was recorded on Agilent 7890 B GC. GCMS data were recorded on Agilent 7890 B GC and 5977 A MSD mass analyser. HRMS data were recorded on a Thermo Scientific Q-Exactive, Accela 1250 pump.

${ }^{1}$ (a) Sato, Y.; Tamura, T.; Kinbara, A.; Morib, M. Adv. Synth. Catal. 2007, 349, 647. (b) Peña, D.; Cobas, A.; Pérez, D.; Guitián, E. Synthesis 2002, 1454.

2 (a) Wagner, A.; Han, W.; Mayer, P.; Ofial, A. R. Adv. Synth. Catal. 2013, 355, 3058. (b) Zhang, L.; Peng, C.; Zhao, D.; Wang, Y.; Fu, H.-J.; Shen, Q.; Li, J.-X. Chem. Commun. 2012, 48, 5928. 


\section{General Procedure for the MCC Involving Arynes, Tertiary Amines and $\mathrm{CO}_{2}$}

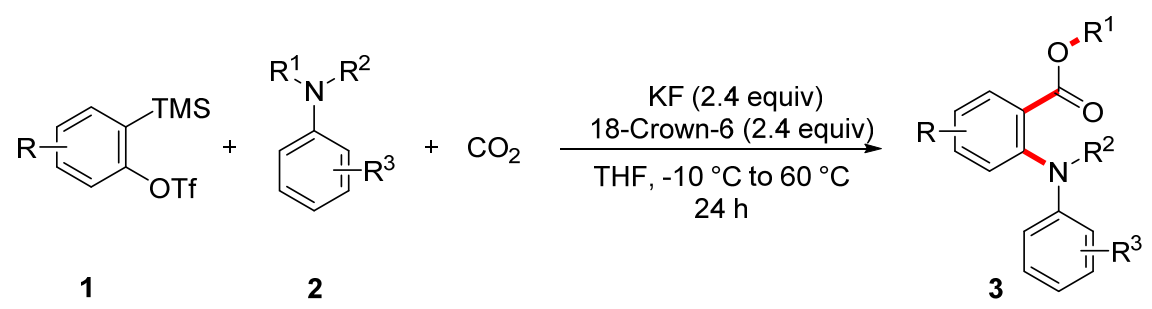

To a flame-dried screw-capped test tube equipped with a magnetic stir bar was added the 18-crown-6 (0.317 g, $1.20 \mathrm{mmol}), \mathrm{KF}(0.070 \mathrm{~g}, 1.20 \mathrm{mmol})$ inside the glove box. The mixture was dissolved in $2.0 \mathrm{~mL}$ of THF outside the glove box under argon. To this mixture was added tertiary amine $2(0.50 \mathrm{mmol})$. The resultant reaction mixture was cooled to $-10{ }^{\circ} \mathrm{C}$ and kept stirring for five minutes under an atmosphere of $\mathrm{CO}_{2}$ (balloon pressure). To the stirring solution was added the aryne precursor $1(0.60 \mathrm{mmol})$. Then the reaction mixture was slowly warmed to $\mathrm{rt}$ and kept stirring for $12 \mathrm{~h}$ under an atmosphere of $\mathrm{CO}_{2}$ (balloon). Next, the screw-capped tube was tightly closed and transferred to a pre-heated oil bath at $60{ }^{\circ} \mathrm{C}$ for $12 \mathrm{~h}$. The reaction mixture was subsequently cooled. The solvent was evaporated and the crude residue was purified by flash column chromatography on silica gel to afford the corresponding 2-arylamino benzoate derivatives $\mathbf{3}$ in good yields.

\section{General Procedure for the MCC Involving Arynes, Electron-poor Tertiary Amines and $\mathrm{CO}_{2}$}

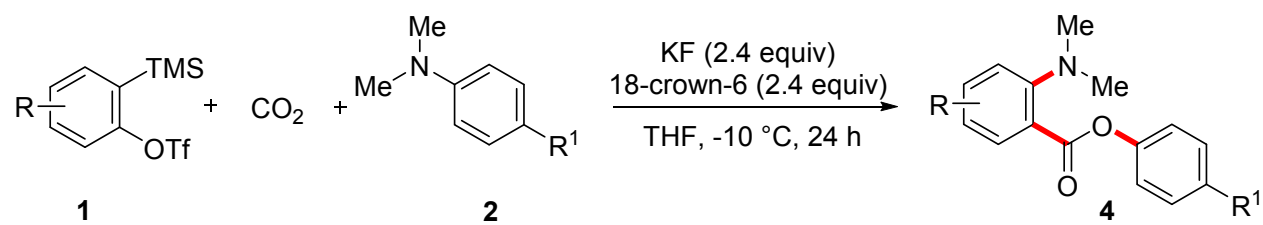

To a flame-dried screw-capped test tube equipped with a magnetic stir bar was added the 18-crown-6 (0.317 g, $1.20 \mathrm{mmol}), \mathrm{KF}(0.070 \mathrm{~g}, 1.20 \mathrm{mmol})$ inside the glove box. The mixture was dissolved in $2.0 \mathrm{~mL}$ of THF outside the glove box under argon. To this mixture was added tertiary amine $2(0.50 \mathrm{mmol})$. The resultant reaction mixture was cooled to $-10{ }^{\circ} \mathrm{C}$ and kept stirring for five minutes under an atmosphere of $\mathrm{CO}_{2}$ (balloon pressure). To the stirring solution was added the aryne precursor $1(0.60 \mathrm{mmol})$ and kept stirring at $-10{ }^{\circ} \mathrm{C}$ for $24 \mathrm{~h}$ under an 
atmosphere of $\mathrm{CO}_{2}$ (balloon). Then the reaction was quenched and the solvent was evaporated. Subsequently, the crude residue was purified by flash column chromatography on silica gel to afford the corresponding 2-aminoaryl benzoate derivatives $\mathbf{4}$ in moderate to good yields.

\section{Mechanistic Experiments}

\section{a) Experiment to Confirm the Formation of Dimethyl Phenyl Ammonium Benzoate (5) as Intermediate in the Reaction}

Isolation of intermediate 5.HOTf

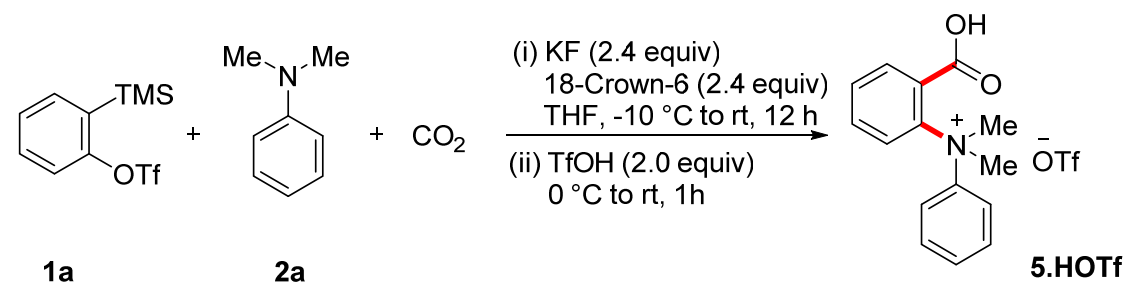

To a flame-dried screw-capped test tube equipped with a magnetic stir bar was added the 18-crown-6 (0.159 g, $0.60 \mathrm{mmol}), \mathrm{KF}(0.035 \mathrm{~g}, 0.60 \mathrm{mmol})$ inside the glove box. The mixture was dissolved in $1.0 \mathrm{~mL}$ of THF outside the glove box under argon. To this mixture was added $N, N$-dimethylaniline $2 \mathbf{a}(0.030 \mathrm{~g}, 32 \mu \mathrm{L}, 0.25 \mathrm{mmol})$. The resultant reaction mixture was cooled to $-10{ }^{\circ} \mathrm{C}$ and kept stirring for five minutes under an atmosphere of $\mathrm{CO}_{2}$ (balloon pressure). To the stirring solution was added 2-(trimethylsilyl)phenyl trifluoromethanesulfonate 1a $(0.090 \mathrm{~g}$, $73 \mu \mathrm{L}, 0.30 \mathrm{mmol}$ ) and the reaction mixture was slowly warmed to $\mathrm{rt}$ and kept stirring for $12 \mathrm{~h}$ under an atmosphere of $\mathrm{CO}_{2}$ (balloon). Then the reaction mixture was quenched with trifluoromethanesulfonic acid $(0.075 \mathrm{~g}, 44 \mu \mathrm{L}, 0.50 \mathrm{mmol})$ at $0{ }^{\circ} \mathrm{C}$ and stirred for $1 \mathrm{~h}$. The reaction mixture was then diluted with $\mathrm{CH}_{2} \mathrm{Cl}_{2}(2.0 \mathrm{~mL})$ and purification was carried out with a short pad of silica gel by eluting first with EtOAc $(10.0 \mathrm{~mL})$ followed by $\mathrm{MeOH}(20.0 \mathrm{~mL})$, which elutes the desired product 2-carboxy- $N, N$-dimethyl- $N$-phenylbenzenaminium trifluoromethanesulfonate 5.HOTf along with 18 -crown-6 as a light green solid $(0.200 \mathrm{~g})$. This mixture was used in the next step without further purification. 


\section{2-Carboxy- $N, N$-dimethyl- $N$-phenylbenzenaminium salt (5.HOTf)}<smiles>C[N+](C)(c1ccccc1)c1ccccc1C(=O)O</smiles>

${ }^{1}$ H NMR (400 MHz, $\left.\mathbf{C D C l}_{3}\right) \delta 8.14(\mathrm{~d}, J=8.6 \mathrm{~Hz}, 1 \mathrm{H}), 7.69-7.65(\mathrm{~m}$, 1H), 7.50-7.49 (m, 2H), 7.37-7.30 (m, 5H), 4.09 (s, 6H). ${ }^{13}$ C NMR (100 $\left.\mathbf{M H z}, \mathbf{C D C l}_{3}\right) \delta 166.37,147.36,144.76,132.60,131.66,130.70,130.35$, $130.12,128.90,125.24,122.06,121.38,118.88,115.70,59.71 .{ }^{19}$ F NMR $\left(376 \mathrm{MHz}, \mathbf{C D C l}_{3}\right) \delta$-78.44. HRMS (ESI) calculated $[\mathrm{M}]^{+}$for $\mathrm{C}_{15} \mathrm{H}_{16} \mathrm{O}_{2} \mathrm{~N}$ : 242.1176, found: 242.1174.

\section{Conversion of 5.HOTf to $3 a$}
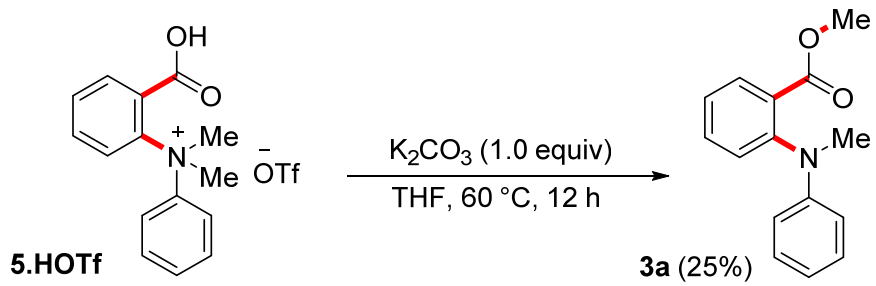

To a round bottom flask containing mixture of 2-carboxy- $N, N$-dimethyl- $N$ phenylbenzenaminium trifluoromethanesulfonate 5.HOTf and 18-crown-6 (0.200 g) was added $\mathrm{K}_{2} \mathrm{CO}_{3}(0.034 \mathrm{~g}, 0.25 \mathrm{mmol})$ at room temperature. Then the mixture was dissolved in THF (1.0 $\mathrm{mL}$ ) under argon atmosphere and round bottom flask kept in a pre-heated oil bath at $60{ }^{\circ} \mathrm{C}$ for 12 h. The reaction mixture cooled and diluted with DCM $(2.0 \mathrm{~mL})$ and filtered through silica pad and eluted with DCM (10.0 mL). The solvent was evaporated to obtain the crude residue, which on column chromatography afforded methyl 2-(methyl(phenyl)amino)benzoate 3a as yellow viscous oil (0.015 g, 25\% yield, for two steps).

This result tends to indicates that the MCCs involving arynes, tertiary amines and $\mathrm{CO}_{2}$ proceeds via the formation of dimethyl phenyl ammonium benzoate intermediate 5 followed by nitrogen to oxygen alkyl group migration (Scheme 5, eq 3 of the manuscript). 
${ }^{1} \mathrm{H}$ and ${ }^{13} \mathrm{C}$ NMR Spectrum of 2-Carboxy- $N, N$-dimethyl- $N$-phenylbenzenaminium salt (5.HOTf)

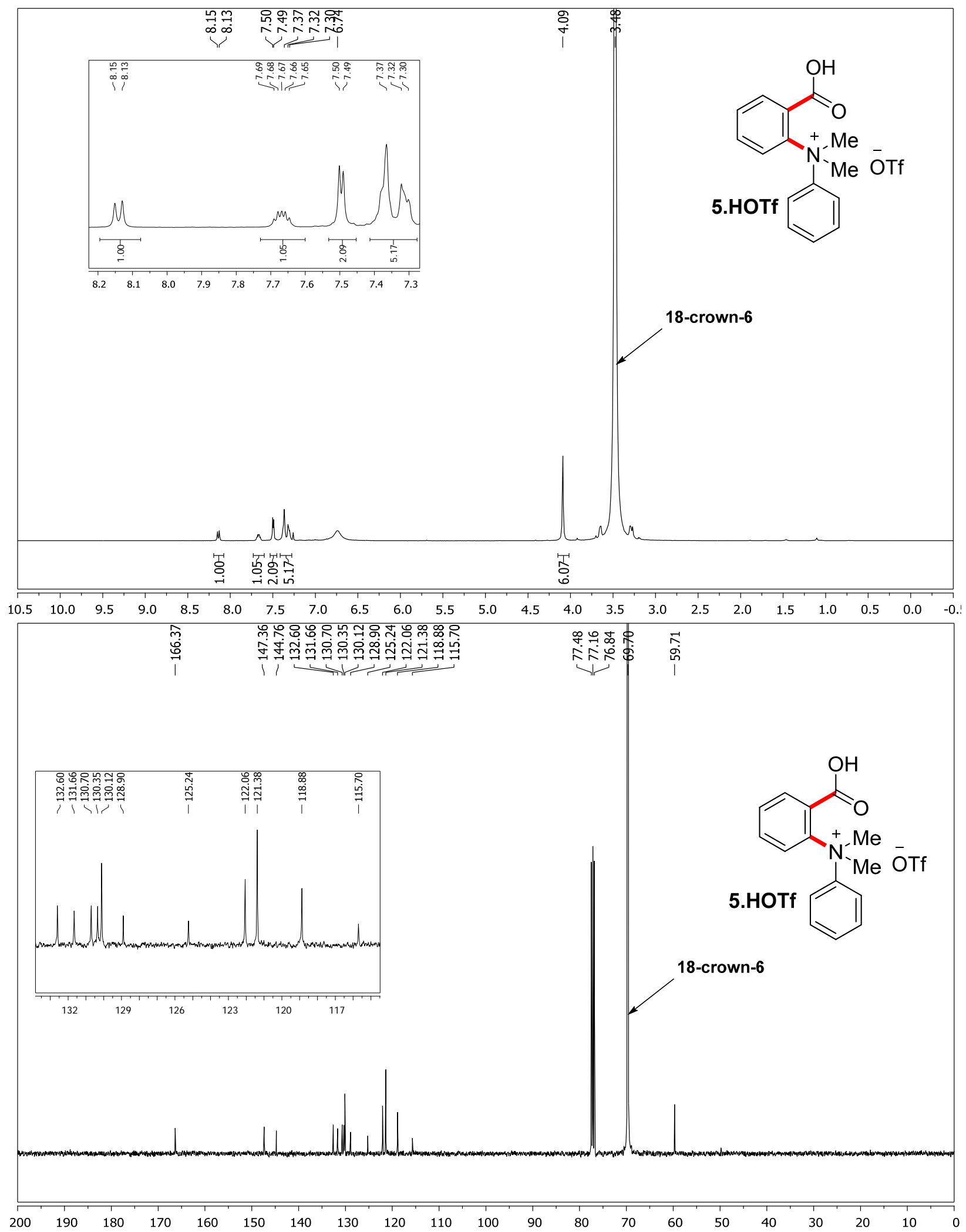




\section{${ }^{19}$ F NMR of Spectrum of 2-Carboxy- $N, N$-dimethyl- $N$-phenylbenzenaminium salt (5.HOTf)}

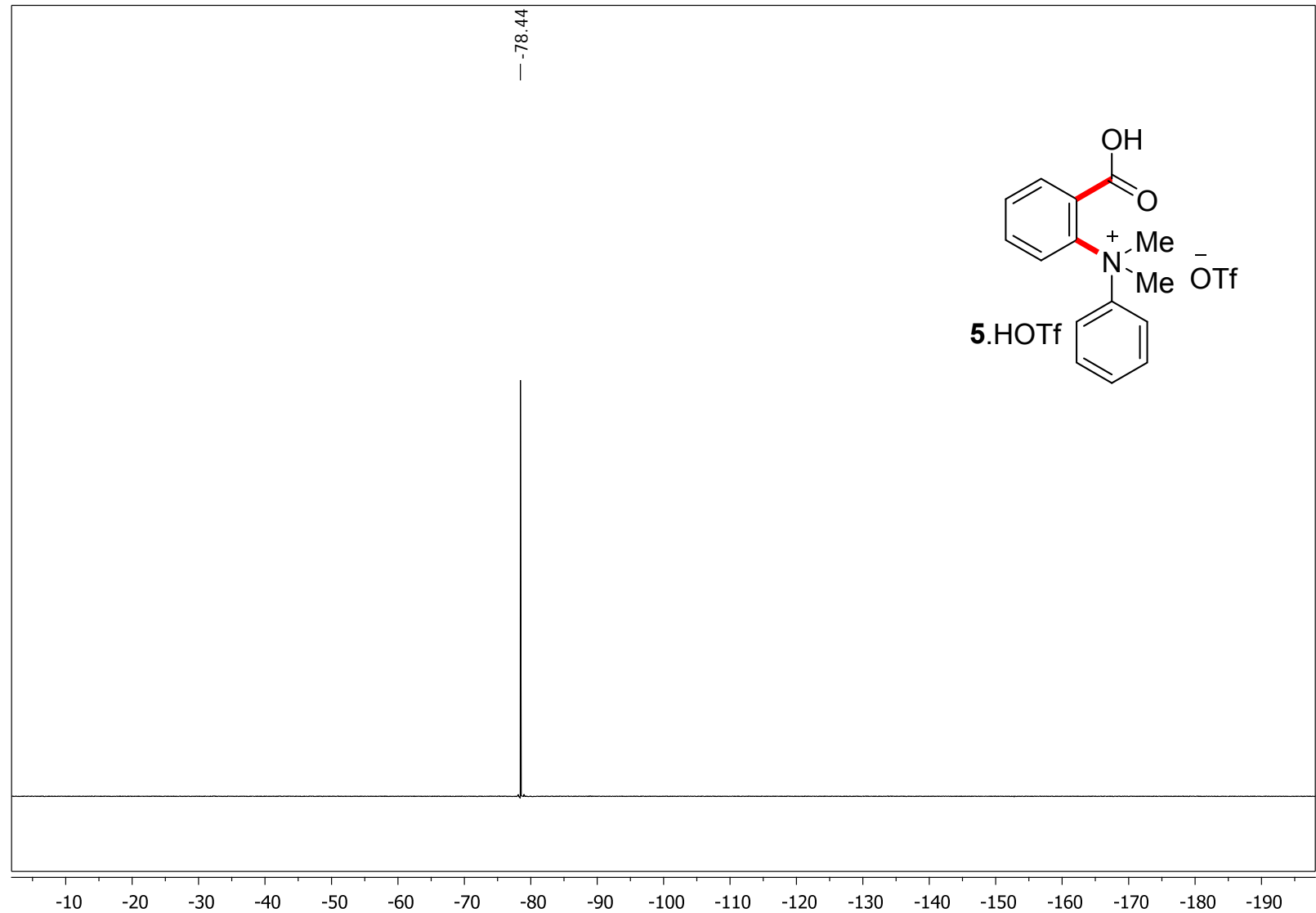

\section{HRMS (ESI) of 2-Carboxy- $N, N$-dimethyl- $N$-phenylbenzenaminium salt (5.HOTf)}

SJ-47 \#98 RT: $0.43 \quad$ AV: $1 \quad$ NL: $4.97 \mathrm{E} 8$ T: FTMS + p ESI Full ms [100.00-1500.00]
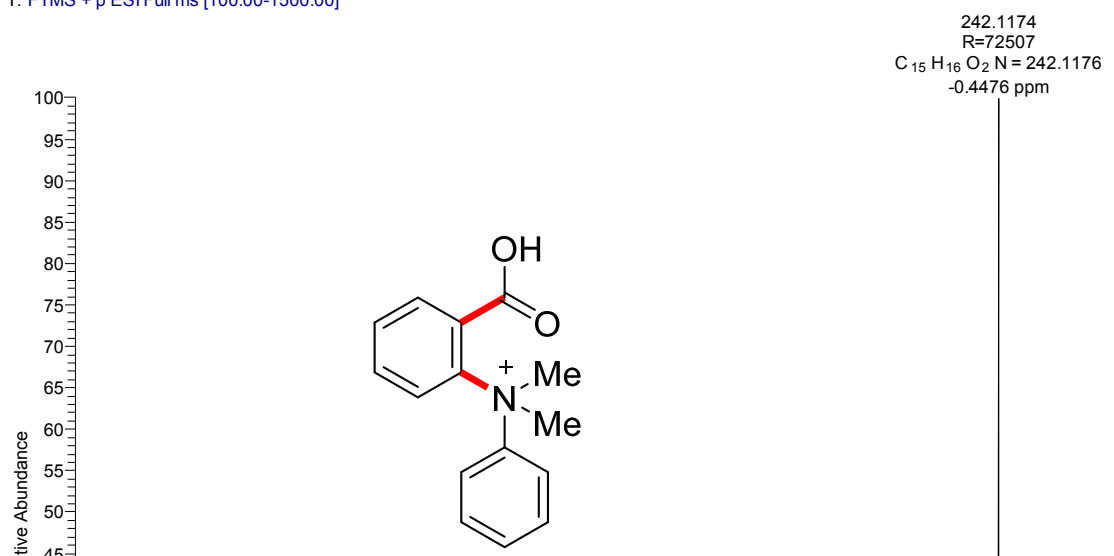

Chemical Formula: $\mathrm{C}_{15} \mathrm{H}_{16} \mathrm{NO}_{2}{ }^{+}$ Exact Mass: 242.1176

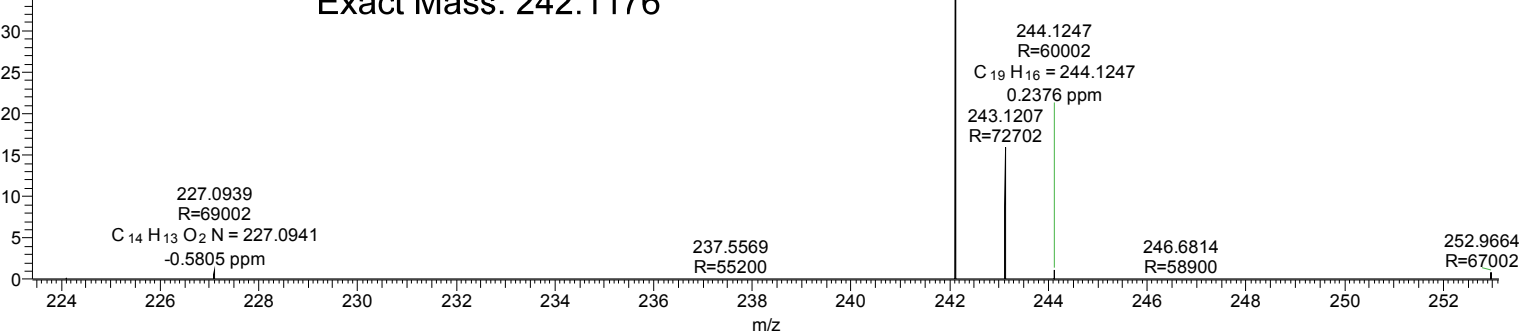




\section{b) Experiments to Probe the Mechanism of the Alkyl Group Migration (Intramolecular or Intermolecular Nucleophilic Substitution)}

\section{Cross-over Experiment: Reaction of aryne, $\mathrm{CO}_{2}$ with a mixture of tertiary amines $2 a$ and $2 \mathrm{~m}$}
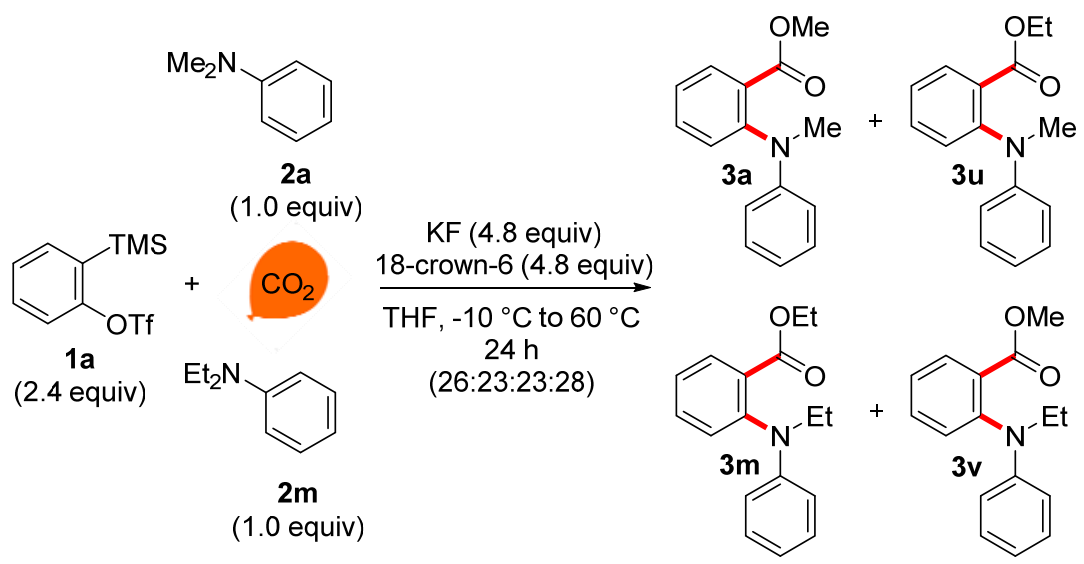

To a flame-dried screw-capped test tube equipped with a magnetic stir bar was added the 18-crown-6 (0.317 g, $1.20 \mathrm{mmol}), \mathrm{KF}(0.070 \mathrm{~g}, 1.20 \mathrm{mmol})$ inside the glove box. The mixture was dissolved in $2.0 \mathrm{~mL}$ of THF outside the glove box under argon. To this mixture was added $N, N$-dimethylaniline $2 \mathbf{a}(0.030 \mathrm{~g}, 32 \mu \mathrm{L}, 0.25 \mathrm{mmol})$, and $N, N$-diethylaniline $2 \mathbf{m}(0.037 \mathrm{~g}, 40$ $\mu \mathrm{L}, 0.25 \mathrm{mmol}$ ). The resultant reaction mixture was cooled to $-10{ }^{\circ} \mathrm{C}$ and kept stirring for five minutes under an atmosphere of $\mathrm{CO}_{2}$ (balloon pressure). To the stirring solution was added 2(trimethylsilyl)phenyl trifluoromethanesulfonate 1a $(0.179 \mathrm{~g}, 146 \mu \mathrm{L}, 0.60 \mathrm{mmol})$. Then the reaction mixture was slowly warmed to $\mathrm{rt}$ and kept stirring for $12 \mathrm{~h}$ under an atmosphere of $\mathrm{CO}_{2}$ (balloon). Next, the screw-capped tube was tightly closed and transferred to a pre-heated oil bath at $60{ }^{\circ} \mathrm{C}$ for $12 \mathrm{~h}$. The reaction mixture was subsequently cooled and diluted with $\mathrm{CH}_{2} \mathrm{Cl}_{2}(2.0$ $\mathrm{mL})$ and filtered through a short pad of silica gel and eluted with $\mathrm{CH}_{2} \mathrm{Cl}_{2}(15.0 \mathrm{~mL})$. The solvent was evaporated to obtain the crude product. GC analysis of crude reaction mixture confirmed the formation of four products methyl 2-(methyl(phenyl)amino)benzoate 3a, ethyl 2(ethyl(phenyl)amino)benzoate $\mathbf{3 m}$, and the cross-over products ethyl 2(methyl(phenyl)amino)benzoate 3u, methyl 2-(ethyl(phenyl)amino)benzoate 3v (Product Selectivity: 3a:3u:3v:3m is 26:23:23:28). 


\section{Attempted MCC of aryne, $\mathrm{CO}_{2}$ and $\mathrm{N}$-ethyl- $\mathrm{N}$-methylaniline (6)}

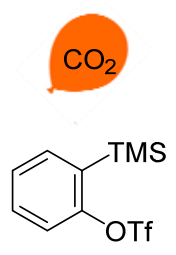

$1 \mathrm{a}$

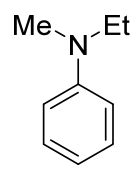

6
KF (4.8 equiv) $\underset{\text { THF },-10{ }^{\circ} \mathrm{C} \text { to } 60^{\circ} \mathrm{C}}{\stackrel{18 \text {-crown- } 6 \text { (4.8 equiv) }}{\longrightarrow}}$

$24 \mathrm{~h}$

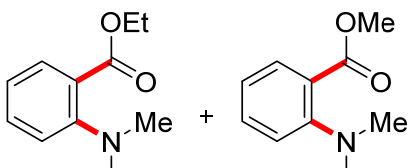<smiles>Cc1ccccc1</smiles>

$3 a$<smiles>Cc1ccccc1</smiles><smiles>CCOC(=O)c1ccccc1N(c1ccccc1)c1ccccc1</smiles>

To a flame-dried screw-capped test tube equipped with a magnetic stir bar was added the 18-crown-6 (0.159 g, $0.60 \mathrm{mmol}), \mathrm{KF}(0.035 \mathrm{~g}, 0.60 \mathrm{mmol})$ inside the glove box. The mixture was dissolved in $1.0 \mathrm{~mL}$ of THF outside the glove box under argon. To this mixture was added $N$-ethyl- $N$-methylaniline 6 ( $0.034 \mathrm{~g}, 0.25 \mathrm{mmol})$. The resultant reaction mixture was cooled to $10{ }^{\circ} \mathrm{C}$ and kept stirring for five minutes under an atmosphere of $\mathrm{CO}_{2}$ (balloon pressure). To the stirring solution was added 2-(trimethylsily))phenyl trifluoromethanesulfonate 1a (0.090 g, 73 $\mu \mathrm{L}, 0.30 \mathrm{mmol})$. Then the reaction mixture was slowly warmed to $\mathrm{rt}$ and kept stirring for $12 \mathrm{~h}$ under an atmosphere of $\mathrm{CO}_{2}$ (balloon). Next, the screw-capped tube was tightly closed and transferred to a pre-heated oil bath at $60{ }^{\circ} \mathrm{C}$ for $12 \mathrm{~h}$. The reaction mixture was subsequently cooled and diluted with $\mathrm{CH}_{2} \mathrm{Cl}_{2}(2.0 \mathrm{~mL})$ and filtered through a short pad of silica gel and eluted with $\mathrm{CH}_{2} \mathrm{Cl}_{2}(10.0 \mathrm{~mL})$. The solvent was evaporated to obtain the crude product. GC and GCMS analysis of crude reaction mixture confirmed the formation of four products ethyl 2(methyl(phenyl)amino)benzoate 3u, methyl 2-(ethyl(phenyl)amino)benzoate $\mathbf{3 v}$ and the crossover products methyl 2-(methyl(phenyl)amino)benzoate 3a, ethyl 2(ethyl(phenyl)amino)benzoate 3m (Product Selectivity: 23:18:33:26). The crude residue was purified by flash column chromatography (Pet. ether $/$ EtOAc $=98 / 02$ ) on silica gel to afford the mixture of four products $\mathbf{3 u}, \mathbf{3 v}, \mathbf{3 a}$, and $\mathbf{3 m}$ as a yellow viscous oil $(0.062 \mathrm{~g})$. 
Ethyl 2-(methyl(phenyl)amino)benzoate (3u), Methyl 2-(ethyl(phenyl)amino)benzoate (3v), Methyl 2-(methyl(phenyl)amino)benzoate (3a), Ethyl 2-(ethyl(phenyl)amino)benzoate (3m)

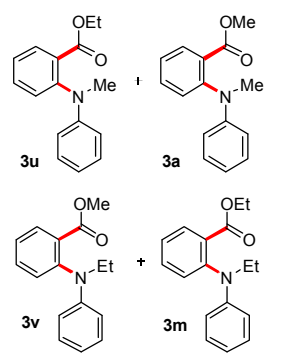

Representative peaks of products ${ }^{1} \mathrm{H}$ NMR (400 $\left.\mathbf{~ M H z}, \mathbf{C D C l}_{3}\right) \delta 4.08$ (q, $J=$ $7.2 \mathrm{~Hz}), 3.33$ (s). ${ }^{13} \mathbf{C}$ NMR (100 $\left.\mathbf{~ M H z}, \mathbf{C D C l}_{\mathbf{3}}\right) \delta$ peaks of ester carbonyl $167.68,167.59,167.30,167.22$, aliphatic carbon peaks $61.12,61.06,52.12$, 52.05, 46.98, 40.45, 40.38, 14.08, 14.05, 13.16, 13.05. HRMS (ESI) calculated $[\mathrm{M}+\mathrm{H}]^{+}$for 3a: $\mathrm{C}_{15} \mathrm{H}_{16} \mathrm{O}_{2} \mathrm{~N}: 242.1176$, found: 242.1172 , for $3 \mathbf{m}: \mathrm{C}_{17} \mathrm{H}_{20} \mathrm{O}_{2} \mathrm{~N}$ : 270.1489, found: 270.1485 , for $3 \mathbf{u}$, and $3 \mathbf{v}: \mathrm{C}_{16} \mathrm{H}_{18} \mathrm{O}_{2} \mathrm{~N}: 256.1332$, found: 256.1329 .

\section{Dilution Experiments: Attempted MCC of aryne, $\mathrm{CO}_{2}$ and $\mathrm{N}$-ethyl- $\mathrm{N}$-methylaniline (6)}

The reaction of $N$-ethyl- $N$-methylaniline 6 was performed under dilute reaction conditions using $5.0 \mathrm{~mL}$ and $10.0 \mathrm{~mL}$ of THF as solvent respectively. Product selectivity determined by $\mathrm{GC}$ analysis of crude reaction mixture.

1. Following the above mentioned procedure, the reaction of aryne generated from $\mathbf{1 a}, \mathrm{CO}_{2}$ and $N$-ethyl- $N$-methylaniline $\mathbf{6}$ in $\mathbf{5 . 0} \mathbf{~ m L}$ of THF as a solvent afforded the four products (3a, $3 \mathbf{u}, \mathbf{3 v}$, and $3 \mathrm{~m}$ ) in the ratio $23: 19: 32: 26$.

2. For MCC of aryne generated from $1 \mathrm{a}, \mathrm{CO}_{2}$ and $N$-ethyl- $N$-methylaniline $\mathbf{6}$ in $\mathbf{1 0 . 0} \mathbf{~ m L}$ of THF as a solvent at $-10{ }^{\circ} \mathrm{C}$ to $\mathrm{rt}$ for $12 \mathrm{~h}$ afforded the expected products $(\mathbf{3 a}, \mathbf{3 u}, \mathbf{3 v}$, and $\mathbf{3 m}$ ) without heating of the reaction mixture in the ratio 24:19:32:25.

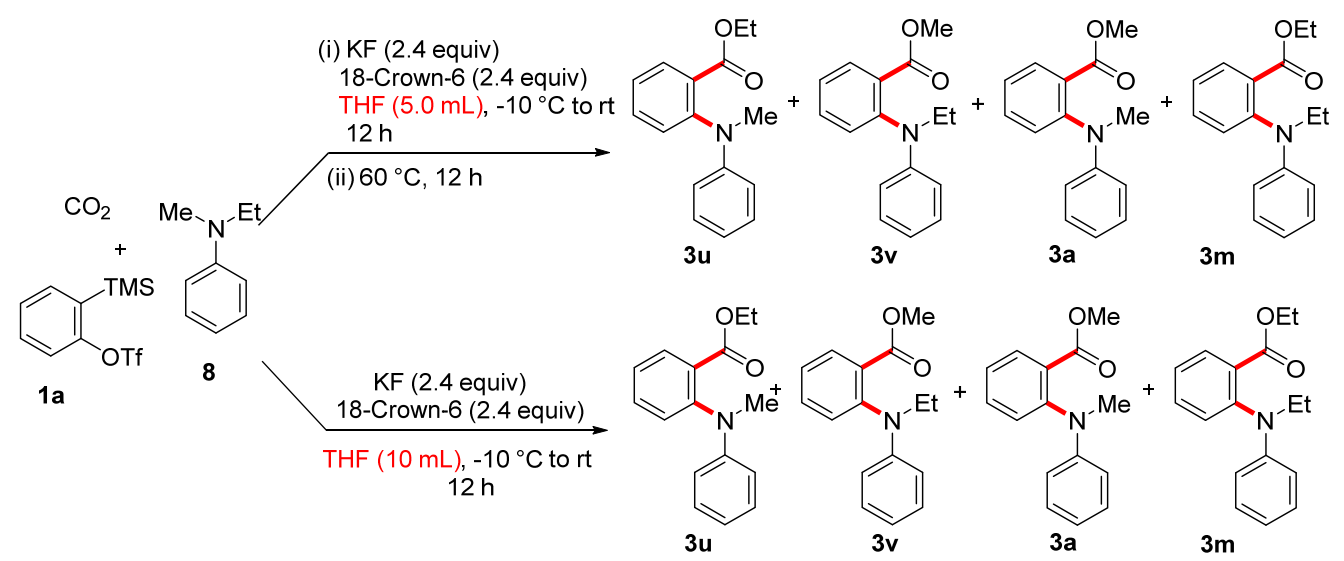

Possibly, the dimethyl phenyl ammonium benzoate intermediate 5 formed in the reaction is soluble upon dilution and facilitating the alkyl group migration. However, when the reactions were performed in $1.0 \mathrm{~mL}$ and $5.0 \mathrm{~mL}$ of THF, quaternary ammonium salt intermediate 5 is not soluble and resulted in the formation of white precipitate in reaction vessel. Therefore, heating is necessary to increase the solubility of $\mathbf{5}$ which finally furnished the desired products. 


\section{GC of Methyl 2-(methyl(phenyl)amino)benzoate (3a)}

Data File: $\quad$ D:LResult 12016 $\quad$ SI-107.rslt LSI-107General method.met2016-06-24 11-24-31 (GMT +05-30).dat

Method: $\quad$ D:LMethodlmi- 55 and 56.met

Acquired: $\quad$ 24/6/2016 11:36:06 AM (GMT +05:30)

Printed: $\quad$ 1/7/2016 4:23:47 PM (GMT +05:30)

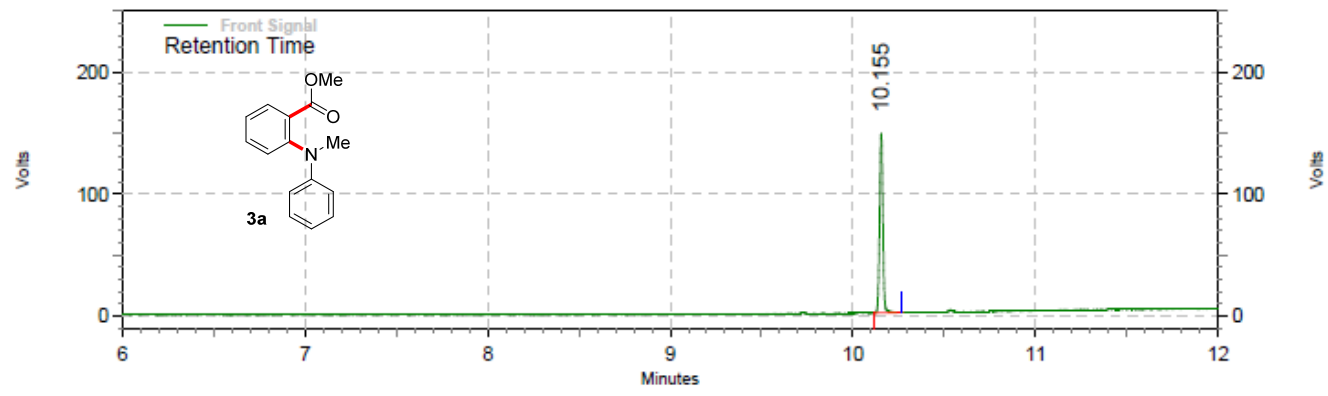

\begin{tabular}{|c|c|c|c|c|}
\hline $\begin{array}{l}\text { Front Signal } \\
\text { Results }\end{array}$ & & & & \\
\hline Retention Time & Area & Area \% & Height & Height $\%$ \\
\hline 10.155 & 1395158 & 100.00 & 1129433 & 100.00 \\
\hline Totals & 1395158 & 100.00 & 1129433 & 100.00 \\
\hline
\end{tabular}

\section{GC of Ethyl 2-(ethyl(phenyl)amino)benzoate (3m)}

Data File: $\quad$ D: Result $12016 \backslash$ SI-108.rslt LSI-108General method.met2016-06-24 11-57-24 (GMT +05-30).dat

Method: $\quad$ D:Lethod $/ \mathrm{mi}-55$ and 56.met

Acquired: $\quad$ 24/6/2016 12:01:18 PM (GMT +05:30)

Printed: $\quad$ 1/7/2016 4:24:21 PM (GMT +05:30)

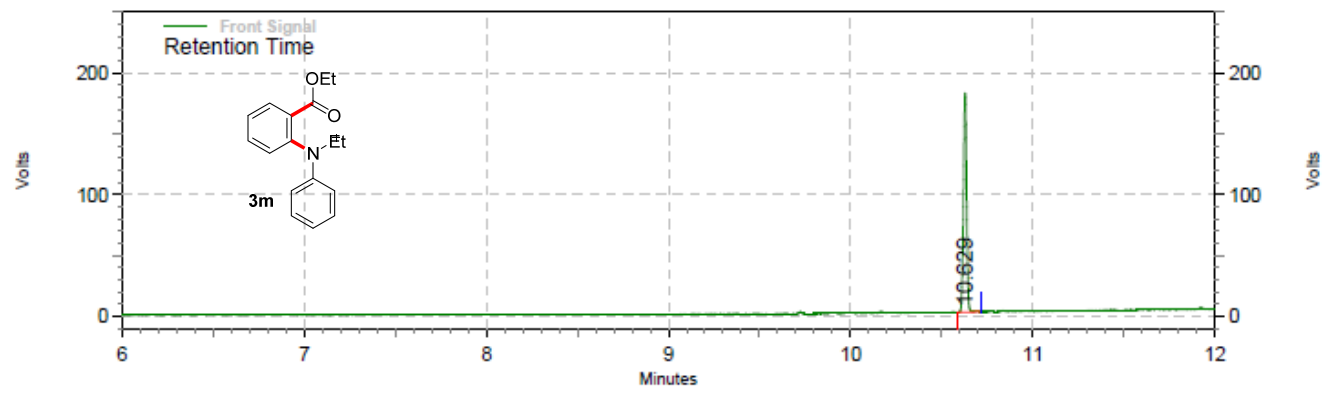

\begin{tabular}{|c|c|c|c|c|}
\hline $\begin{array}{l}\text { Front Signal } \\
\text { Results } \\
\text { Retention Time }\end{array}$ & & & & \\
\hline 10.629 & 1678889 & 100.00 & 1382403 & 100.00 \\
\hline \multicolumn{5}{|l|}{ Totals } \\
\hline & 1678889 & 100.00 & 1382403 & 100.00 \\
\hline
\end{tabular}




\section{GC of Crude Reaction Mixture of Cross-over Experiment}
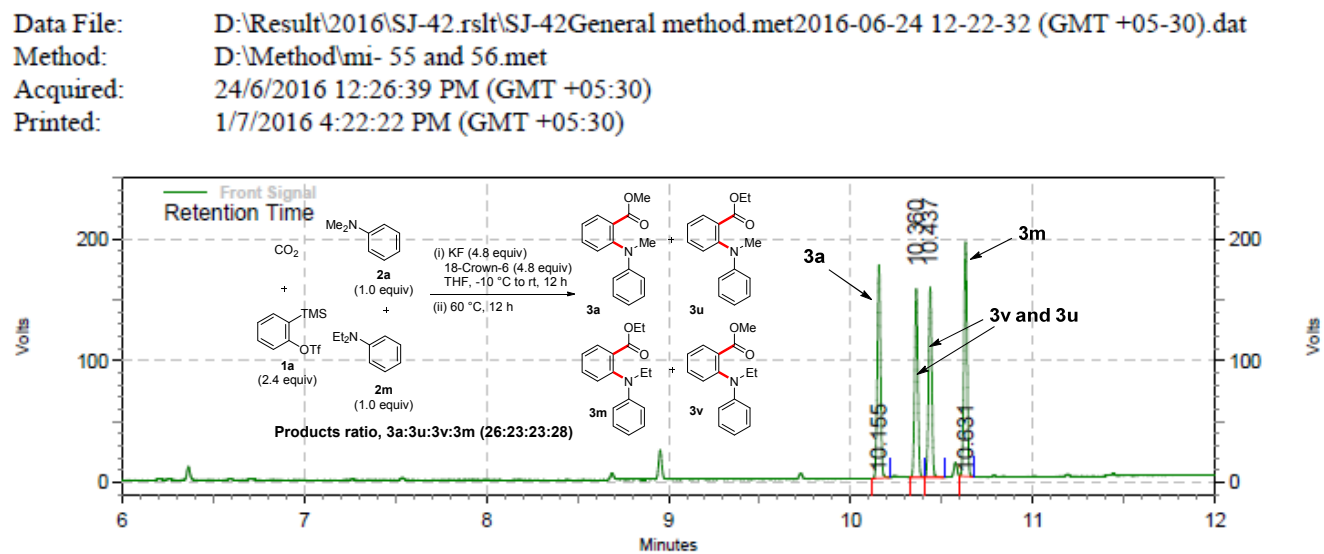

Front Signal

Results

\begin{tabular}{rrrrr} 
Retention Time & Area & Area $\%$ & Height & Height \% \\
\hline 10.155 & 1663979 & 26.23 & 1347450 & 25.83 \\
10.360 & 1427798 & 22.51 & 1191091 & 22.83 \\
10.437 & 1471515 & 23.20 & 1199045 & 22.98 \\
10.631 & 1780513 & 28.07 & 1479467 & 28.36 \\
\hline Totals & & & & \\
& & & & \\
\hline
\end{tabular}

\section{GC of Crude Reaction Mixture in $1.0 \mathrm{~mL}$ THF as Solvent}

Data File: $\quad$ D:LResult 2016 $\quad$ SJ-46.rslt ISJ-46General method.met2016-06-29 14-38-45 (GMT +05-30).dat Method: $\quad$ D:LMethod $\quad$ mi- 55 and 56.met

Acquired: $\quad$ 29/6/2016 2:50:21 PM (GMT +05:30)

Printed: $\quad$ 1/7/2016 4:21:25 PM (GMT +05:30)

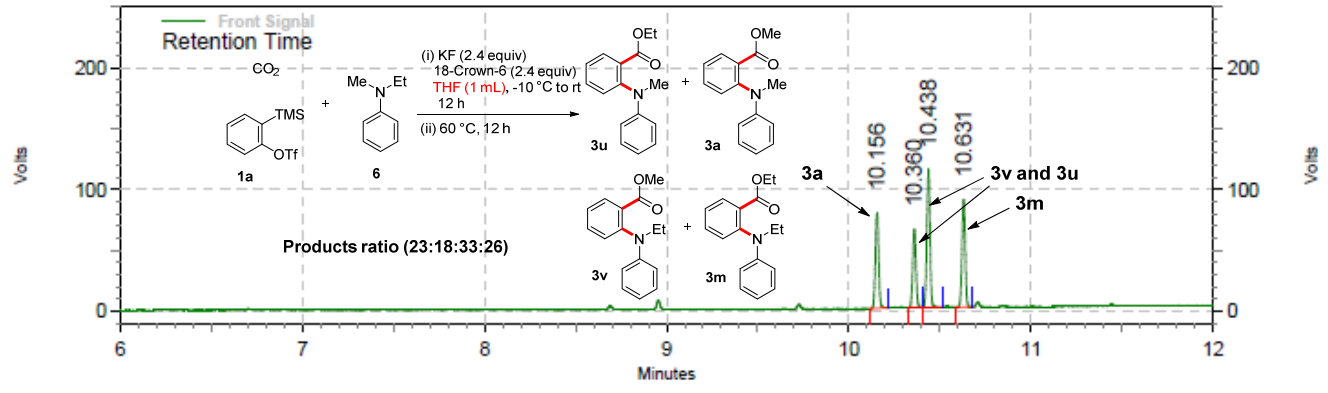

\begin{tabular}{|c|c|c|c|c|}
\hline $\begin{array}{l}\text { Front Signal } \\
\text { Results } \\
\quad \text { Retention Time }\end{array}$ & Area & Area \% & Height & Height \% \\
\hline 10.156 & 754258 & 23.07 & 601828 & 22.72 \\
\hline 10.360 & 603501 & 18.46 & 494009 & 18.65 \\
\hline 10.438 & 1064211 & 32.55 & 873005 & 32.96 \\
\hline 10.631 & 847757 & 25.93 & 680131 & 25.68 \\
\hline Totals & & & & \\
\hline & 3269727 & 100.00 & 2648973 & 100.00 \\
\hline
\end{tabular}




\section{GC of Crude Reaction Mixture in $5.0 \mathrm{~mL}$ THF as Solvent}

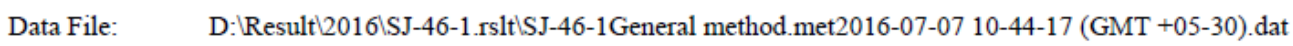

$\begin{array}{ll}\text { Method: } & \text { D:lMethod } \backslash \mathrm{mi}-55 \text { and } 56 . \mathrm{met} \\ \text { Acquired: } & 7 / 7 / 201610: 55: 53 \mathrm{AM}(\mathrm{GMT}+05: 30) \\ \text { Printed: } & 7 / 7 / 201612: 08: 34 \mathrm{PM}(\mathrm{GMT}+05: 30)\end{array}$

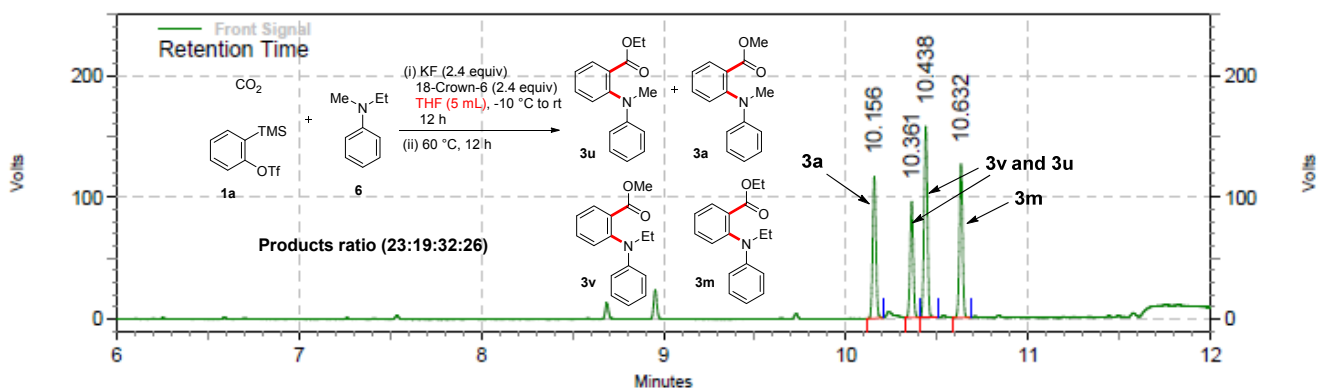

Front Signal

Results

Retention Time

10.156

10.361

10.438

10.632

Totals

\begin{tabular}{rrrr} 
Area & Area $\%$ & Height & Height \% \\
\hline 1104652 & 23.52 & 894691 & 23.54 \\
870184 & 18.53 & 731557 & 19.25 \\
1520371 & 32.37 & 1206155 & 31.74 \\
1200968 & 25.57 & 968018 & 25.47
\end{tabular}

\begin{tabular}{|lllll|}
\hline Totals & & & & \\
& 4696175 & 100.00 & 3800421 & 100.00 \\
\hline
\end{tabular}

\section{GC of Crude Reaction Mixture in $10.0 \mathrm{~mL}$ THF as Solvent}

Data File: $\quad$ D: $\quad$ Result $2016 \backslash$ SJ-46-2.rslt LSJ-46-2General method.met2016-07-07 11-17-12 (GMT +05-30).dat

Method: $\quad$ D:LMethod $\quad$ mi- 55 and 56.met

Acquired: $\quad$ 7/7/2016 11:21:05 AM (GMT +05:30)

Printed: $\quad$ 7/7/2016 12:10:31 PM (GMT +05:30)

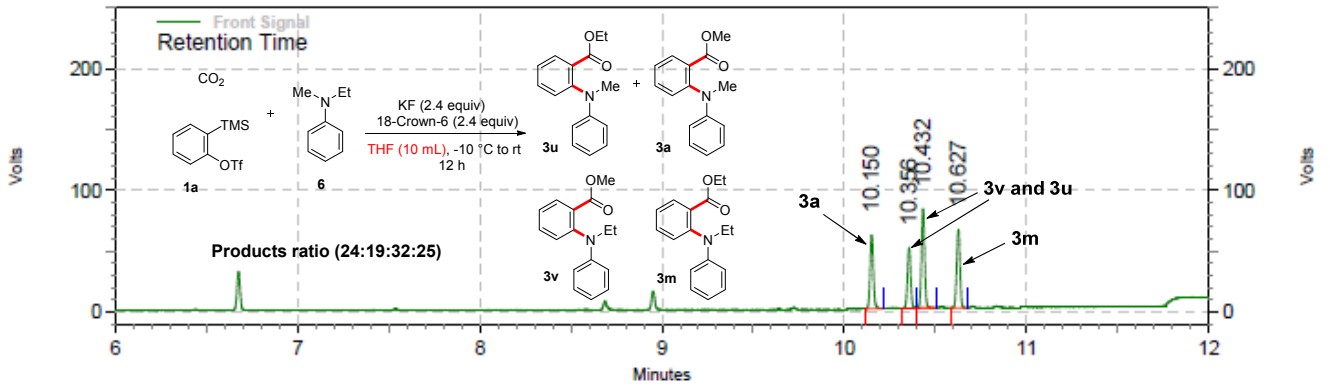

Front Signal Results

Retention Time

10.150

10.356

10.432

10.627

Area Area \% Height Height \%

$\begin{array}{llll}584459 & 23.97 & 465478 & 23.63 \\ 469835 & 19.27 & 382214 & 19.40 \\ 768292 & 31.51 & 626601 & 31.81 \\ 615904 & 25.26 & 495422 & 25.15\end{array}$

Totals 


\section{GCMS Analysis of Crude Reaction Mixture in $1.0 \mathrm{~mL}$ THF as Solvent}

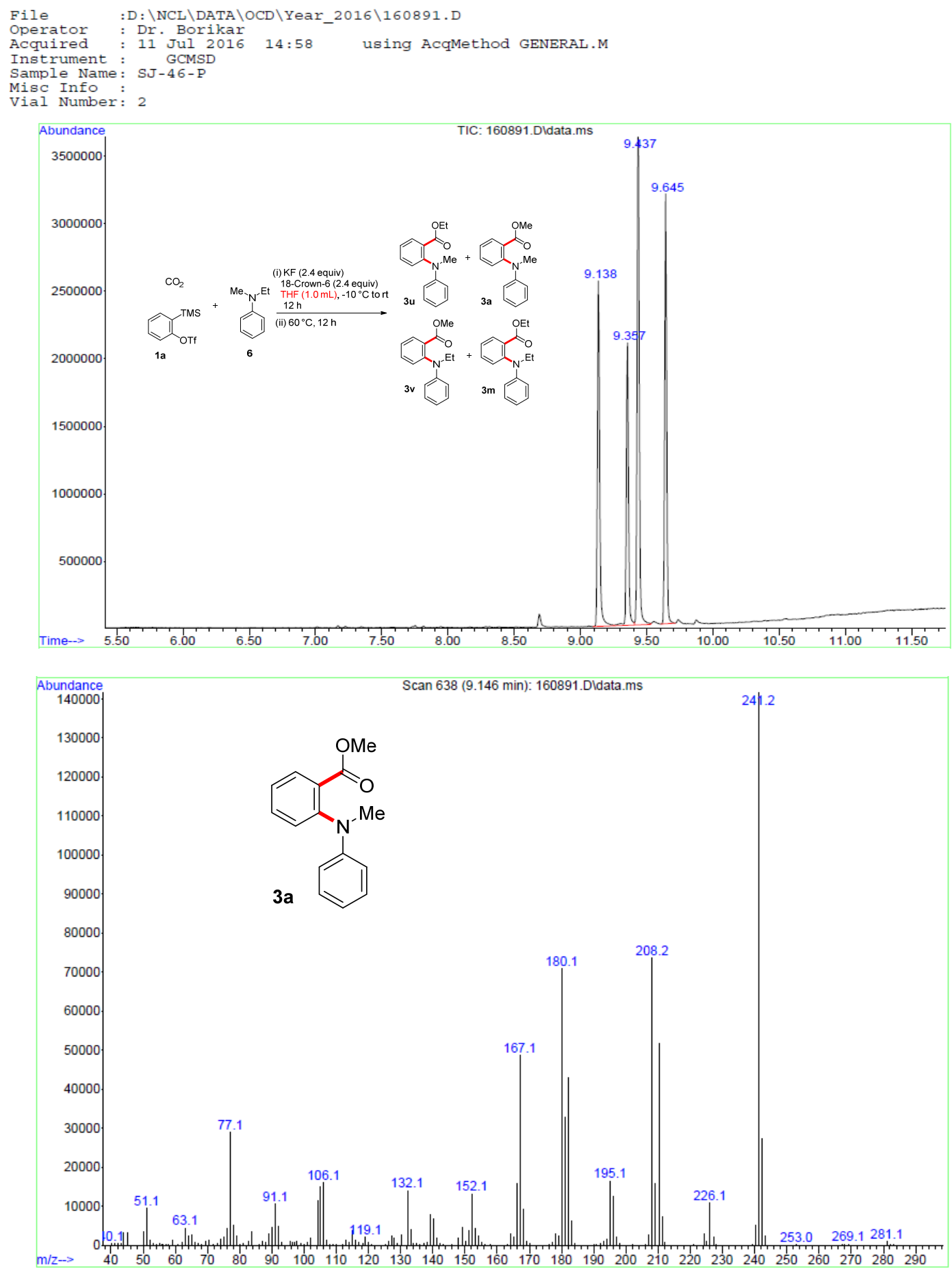



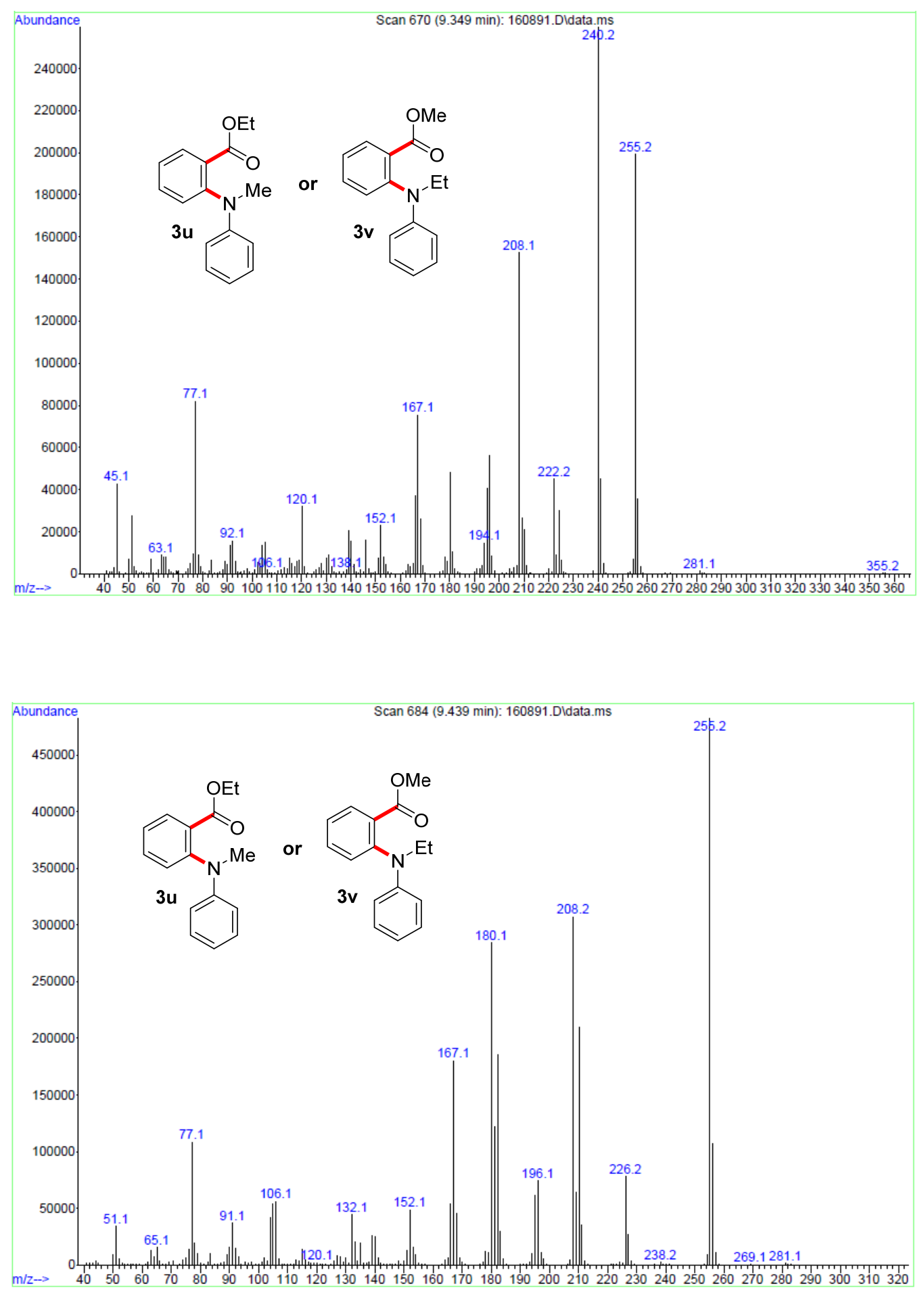


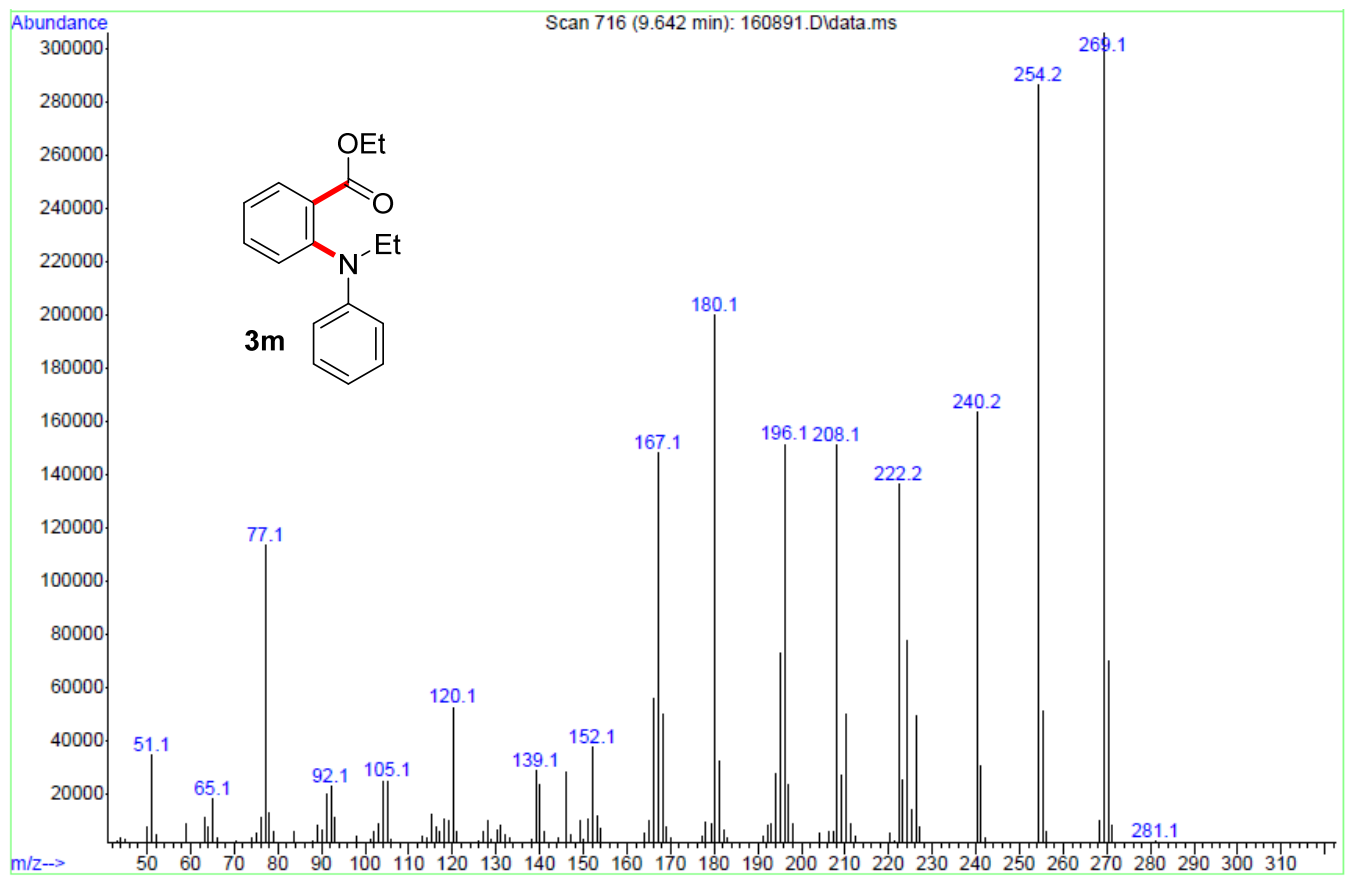

These experiments tend to indicate the possibility of present alkyl group migration in an intermolecular pathway. 
${ }^{1} \mathrm{H}$ and ${ }^{13} \mathrm{C}$ NMR Spectrum of Ethyl 2-(methyl(phenyl)amino)benzoate (3u), Methyl 2(ethyl(phenyl)amino)benzoate (3v), Methyl 2-(methyl(phenyl)amino)benzoate (3a), Ethyl 2(ethyl(phenyl)amino)benzoate $(3 \mathrm{~m})\left(\mathrm{CDCl}_{3}\right)$
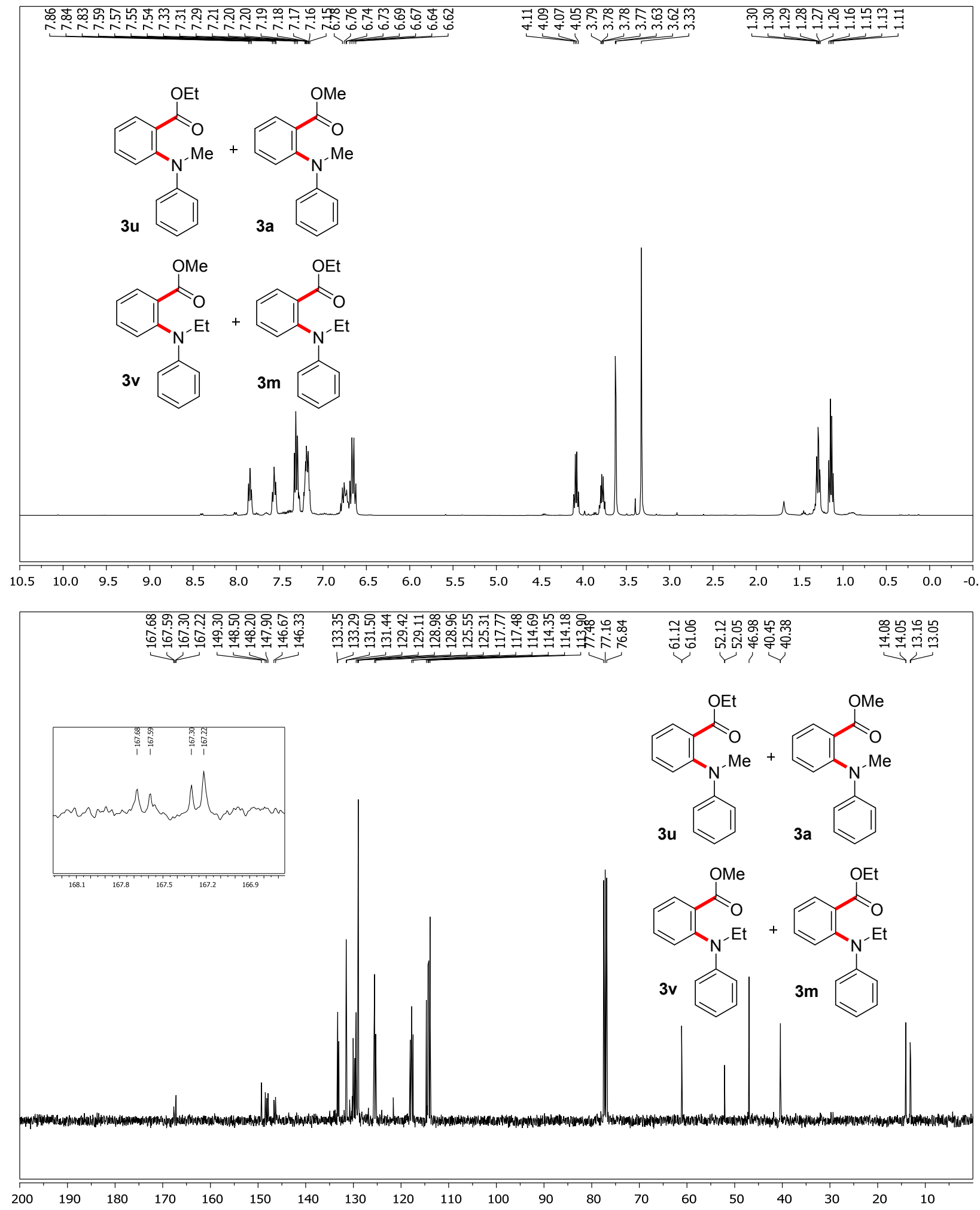


\section{Synthesis and Characterization of 2-Arylamino Benzoate Derivatives}

\section{Methyl 2-(methyl(phenyl)amino)benzoate (3a) ${ }^{3}$}

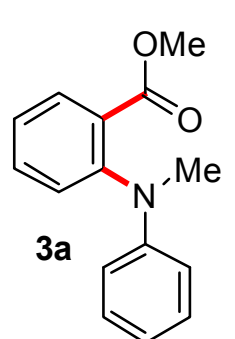

Following the general procedure, 2-(trimethylsilyl)phenyl trifluoromethane sulfonate $1 \mathrm{a}(0.179 \mathrm{~g}, 146 \mu \mathrm{L}, 0.60 \mathrm{mmol})$ was treated with $N, N$-dimethylaniline 2a $(0.061 \mathrm{~g}, 64 \mu \mathrm{L}, 0.50 \mathrm{mmol})$ in the presence of $\mathrm{KF}(0.070 \mathrm{~g}, 1.20 \mathrm{mmol})$ and 18-crown-6 (0.317 g, $1.20 \mathrm{mmol})$ in THF $(2.0 \mathrm{~mL})$ under an atmosphere of $\mathrm{CO}_{2}$ (balloon pressure) at $-10{ }^{\circ} \mathrm{C}$ to $\mathrm{rt}$ for $12 \mathrm{~h}$. Then the reaction mixture was kept stirring at $60{ }^{\circ} \mathrm{C}$ for $12 \mathrm{~h}$ followed by flash column chromatography (Pet. ether $/ \mathrm{EtOAc}=98 / 02$ ) of the crude reaction mixture using silica gel to afford methyl 2-(methyl(phenyl)amino)benzoate 3a as a yellow viscous oil ( $0.105 \mathrm{~g}, 87 \%$ yield $)$.

$\boldsymbol{R}_{\mathbf{f}}\left(\right.$ Pet. ether $/$ EtOAc $=$ 95/05): 0.48; ${ }^{1} \mathbf{H}$ NMR $\left(\mathbf{4 0 0} \mathbf{~ M H z}, \mathbf{C D C l}_{\mathbf{3}}\right) \delta 7.74(\mathrm{~d}, J=7.6 \mathrm{~Hz}, 1 \mathrm{H})$, 7.47 (t, $J=7.5 \mathrm{~Hz}, 1 \mathrm{H}), 7.24-7.18(\mathrm{~m}, 2 \mathrm{H}), 7.13-7.09(\mathrm{~m}, 2 \mathrm{H}), 6.68(\mathrm{t}, J=7.2 \mathrm{~Hz}, 1 \mathrm{H}), 6.59$ (d, $J=8.0 \mathrm{~Hz}, 2 \mathrm{H}), 3.53$ (s, 3H), 3.23 (s, 3H). ${ }^{13} \mathbf{C}$ NMR (100 MHz, $\left.\mathbf{C D C l}_{3}\right) \delta 167.57,149.32$, 148.19, 133.34, 131.49, 129.34, 129.10, 128.97, 125.30, 118.07, 114.34, 52.12, 40.44. HRMS (ESI) calculated $[\mathrm{M}+\mathrm{H}]^{+}$for $\mathrm{C}_{15} \mathrm{H}_{16} \mathrm{O}_{2} \mathrm{~N}: 242.1176$, found: 242.1175 . FTIR $\left(\mathbf{c m}^{-1}\right)$ : 3020,1722 , 1594, 1494, 1345, 1293, 1250, 1224, 759.

\section{Methyl 2-(methyl(p-tolyl)amino)benzoate (3b)}

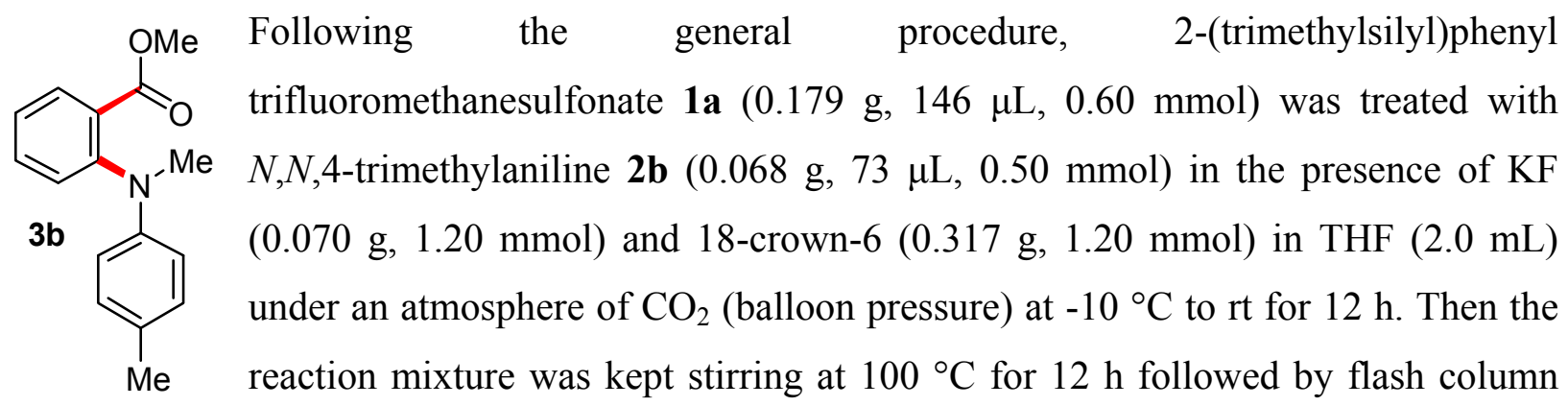
chromatography (Pet. ether $/$ EtOAc $=98 / 02$ ) of the crude reaction mixture using silica gel to afford methyl 2-(methyl( $p$-tolyl)amino)benzoate $\mathbf{3 b}$ as a yellow viscous oil ( $0.092 \mathrm{~g}, 72 \%$ yield). $\boldsymbol{R}_{\mathbf{f}}($ Pet. ether $/$ EtOAc $=95 / 05): 0.49 ;{ }^{1} \mathbf{H}$ NMR $\left(\mathbf{4 0 0} \mathbf{~ M H z}, \mathbf{C D C l}_{3}\right) \delta 7.75(\mathrm{~d}, J=7.7 \mathrm{~Hz}, 1 \mathrm{H})$, 7.49 (t, $J=7.4 \mathrm{~Hz}, 1 \mathrm{H}), 7.25$ (d, $J=7.8 \mathrm{~Hz}, 1 \mathrm{H}), 7.20$ (t, $J=7.5 \mathrm{~Hz}, 1 \mathrm{H}), 6.97$ (d, $J=8.1 \mathrm{~Hz}$, 2H), $6.59(\mathrm{~d}, J=8.2 \mathrm{~Hz}, 2 \mathrm{H}), 3.58(\mathrm{~s}, 3 \mathrm{H}), 3.26(\mathrm{~s}, 3 \mathrm{H}), 2.23(\mathrm{~s}, 3 \mathrm{H}) .{ }^{13} \mathbf{C} \mathbf{~ N M R}(\mathbf{1 0 0} \mathbf{~ M H z}$

\footnotetext{
${ }^{3}$ Bhojgude, S. S.; Kaicharla, T.; Biju, A. T. Org. Lett. 2013, 15, 5452.
} 
$\left.\mathbf{C D C l}_{3}\right) \delta 167.90,148.59,147.29,133.14,131.39,129.55,128.83,128.11,127.76,124.59$, 115.29, 52.12, 40.74, 20.51. HRMS (ESI) calculated $[\mathrm{M}+\mathrm{H}]^{+}$for $\mathrm{C}_{16} \mathrm{H}_{18} \mathrm{O}_{2} \mathrm{~N}: 256.1332$, found: 256.1332. FTIR (cm $\left.{ }^{-1}\right): 3019,2948,2813,2403,1719,1604,1506,1446,1220,1086,760$.

\section{Methyl 2-((4-methoxyphenyl)(methyl)amino)benzoate (3c)}

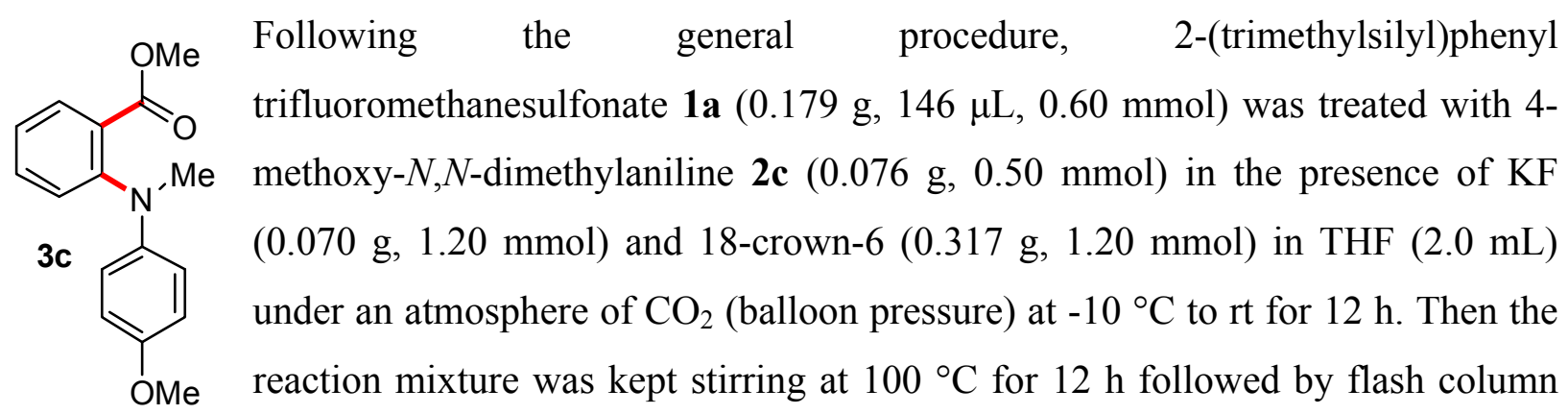
chromatography (Pet. ether $/$ EtOAc $=96 / 04$ ) of the crude reaction mixture using silica gel to afford methyl 2-((4-methoxyphenyl)(methyl)amino)benzoate 3c as a yellow viscous oil (0.132 g, $97 \%$ yield).

$\boldsymbol{R}_{\mathbf{f}}($ Pet. ether $/$ EtOAc $=90 / 10): 0.40 ;{ }^{1} \mathbf{H}$ NMR $\left(\mathbf{4 0 0} \mathbf{~ M H z}, \mathbf{C D C l}_{3}\right) \delta 7.68(\mathrm{~d}, J=7.7 \mathrm{~Hz}, 1 \mathrm{H})$, $7.47(\mathrm{t}, J=7.6 \mathrm{~Hz}, 1 \mathrm{H}), 7.22$ (d, $J=8.1 \mathrm{~Hz}, 1 \mathrm{H}), 7.13$ (t, $J=7.6 \mathrm{~Hz}, 1 \mathrm{H}), 6.78$ (d, $J=9.0 \mathrm{~Hz}$, 2H), $6.72(\mathrm{~d}, J=9.0 \mathrm{~Hz}, 2 \mathrm{H}), 3.74(\mathrm{~s}, 3 \mathrm{H}), 3.57$ (s, 3H), 3.27 (s, 3H). ${ }^{13} \mathbf{C}$ NMR (100 MHz, $\left.\mathbf{C D C l}_{3}\right) \delta 168.14,153.30,148.86,143.89,132.86,131.18,127.67,126.09,123.44,118.20$, 114.48, 55.68, 51.97, 41.34. HRMS (ESI) calculated $[\mathrm{M}+\mathrm{H}]^{+}$for $\mathrm{C}_{16} \mathrm{H}_{18} \mathrm{O}_{3} \mathrm{~N}: 272.1281$, found: 272.1280. FTIR (cm $\left.{ }^{-1}\right): 3019,1720,1510,1489,1241,1216,1182,772$.

\section{Methyl 2-((4-fluorophenyl)(methyl)amino)benzoate (3d)}<smiles>COC(=O)c1ccccc1N(C)c1ccc(F)cc1</smiles>

Following the general procedure, 2-(trimethylsilyl)phenyl trifluoromethanesulfonate $1 \mathrm{a}(0.179 \mathrm{~g}, 146 \mu \mathrm{L}, 0.60 \mathrm{mmol})$ was treated with 4fluoro- $N, N$-dimethylaniline $2 \mathbf{d}(0.070 \mathrm{~g}, 0.50 \mathrm{mmol})$ in the presence of $\mathrm{KF}$ $(0.070 \mathrm{~g}, 1.20 \mathrm{mmol})$ and 18 -crown-6 $(0.317 \mathrm{~g}, 1.20 \mathrm{mmol})$ in THF $(2.0 \mathrm{~mL})$ under an atmosphere of $\mathrm{CO}_{2}$ (balloon pressure) at $-10{ }^{\circ} \mathrm{C}$ to $\mathrm{rt}$ for $12 \mathrm{~h}$. Then the reaction mixture was kept stirring at $100{ }^{\circ} \mathrm{C}$ for $12 \mathrm{~h}$ followed by flash column chromatography (Pet. ether $/ \mathrm{EtOAc}=98 / 02$ ) of the crude reaction mixture using silica gel to afford methyl 2-((4-fluorophenyl)(methyl)amino)benzoate 3d as a yellow viscous oil (0.122 $\mathrm{g}$, $94 \%$ yield). 
$\boldsymbol{R}_{\mathbf{f}}($ Pet. ether $/$ EtOAc $=95 / 05): 0.48 ;{ }^{1} \mathbf{H}$ NMR (400 MHz, $\left.\mathbf{C D C l}_{3}\right) \delta 7.81(\mathrm{~d}, J=7.7 \mathrm{~Hz}, 1 \mathrm{H})$, $7.57(\mathrm{t}, J=7.7 \mathrm{~Hz}, 1 \mathrm{H}), 7.32-7.26(\mathrm{~m}, 2 \mathrm{H}), 6.94-6.90(\mathrm{~m}, 2 \mathrm{H}), 6.67-6.63(\mathrm{~m}, 2 \mathrm{H}), 3.65(\mathrm{~s}, 3 \mathrm{H})$, 3.32 (s, 3H). ${ }^{13} \mathbf{C}$ NMR (100 MHz, $\left.\mathbf{C D C l}_{3}\right) \delta 167.60,156.37$ (d, $\left.J=237.0 \mathrm{~Hz}\right), 148.42,146.01$, 133.32, 131.49, 128.85, 128.27, 124.99, 116.05 (d, $J=7.5 \mathrm{~Hz}), 115.42$ (d, $J=22.3 \mathrm{~Hz}), 52.12$, 40.98. HRMS (ESI) calculated $[\mathrm{M}+\mathrm{H}]^{+}$for $\mathrm{C}_{15} \mathrm{H}_{15} \mathrm{O}_{2} \mathrm{NF}: 260.1081$, found: 260.1081. FTIR $\left(\mathbf{c m}^{-1}\right): 2943,1724,1599,1503,1344,1290,1228,1128,816,761,713$.

\section{Methyl 2-(methyl(m-tolyl)amino)benzoate (3e)}<smiles>COC(=O)c1ccccc1N(C)c1cccc(C)c1</smiles>

Following the general procedure, 2-(trimethylsilyl)phenyl trifluoromethanesulfonate $1 \mathrm{a}(0.179 \mathrm{~g}, 146 \mu \mathrm{L}, 0.60 \mathrm{mmol})$ was treated with $N, N, 3$-trimethylaniline $2 \mathrm{e}(0.068 \mathrm{~g}, 72 \mu \mathrm{L}, 0.50 \mathrm{mmol})$ in the presence of $\mathrm{KF}$ $(0.070 \mathrm{~g}, 1.20 \mathrm{mmol})$ and 18 -crown-6 $(0.317 \mathrm{~g}, 1.20 \mathrm{mmol})$ in THF $(2.0 \mathrm{~mL})$ under an atmosphere of $\mathrm{CO}_{2}$ (balloon pressure) at $-10{ }^{\circ} \mathrm{C}$ to $\mathrm{rt}$ for $12 \mathrm{~h}$. Then the reaction mixture was kept stirring at $60{ }^{\circ} \mathrm{C}$ for $12 \mathrm{~h}$ followed by flash column chromatography (Pet. ether $/$ EtOAc $=98 / 02)$ of the crude reaction mixture using silica gel to afford methyl 2-(methyl( $m$-tolyl)amino)benzoate $\mathbf{3 e}$ as a yellow viscous oil $(0.110 \mathrm{~g}, 86 \%$ yield).

$\boldsymbol{R}_{\mathbf{f}}($ Pet. ether $/$ EtOAc $=95 / 05): 0.54 ;{ }^{1} \mathbf{H}$ NMR (400 $\left.\mathbf{M H z}, \mathbf{C D C l}_{3}\right) \delta 7.84(\mathrm{~d}, J=7.8 \mathrm{~Hz}, 1 \mathrm{H})$, $7.56(\mathrm{t}, J=7.6 \mathrm{~Hz}, 1 \mathrm{H}), 7.33-7.27(\mathrm{~m}, 2 \mathrm{H}), 7.10(\mathrm{t}, J=7.7 \mathrm{~Hz}, 1 \mathrm{H}), 6.62(\mathrm{~d}, J=7.4 \mathrm{~Hz}, 1 \mathrm{H})$, 6.52-6.49 (m, 2H), 3.65 (s, 3H), 3.32 (s, 3H), 2.29 (s, 3H). ${ }^{13} \mathbf{C}$ NMR (100 MHz, CDCl 3 ) $\delta$ 167.56, 149.27, 148.25, 138.58, 133.21, 131.39, 129.24, 128.94, 128.79, 125.10, 119.06, 115.09, 111.71, 52.05, 40.47, 21.79. HRMS (ESI) calculated $[\mathrm{M}+\mathrm{H}]^{+}$for $\mathrm{C}_{16} \mathrm{H}_{18} \mathrm{O}_{2} \mathrm{~N}$ : 256.1332, found: 256.1332. FTIR (cm $\left.{ }^{-1}\right):$ 3018, 1723, 1595, 1490, 1446, 1344, 1292, 1130, 1085, 760.

\section{Methyl 2-((3-bromophenyl)(methyl)amino)benzoate (3f)}

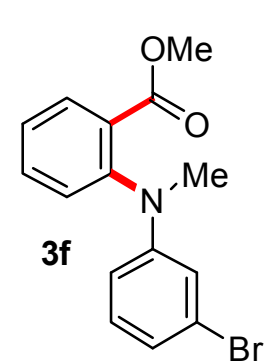

Following the general procedure, 2-(trimethylsilyl)phenyl trifluoromethanesulfonate 1a $(0.179 \mathrm{~g}, 146 \mu \mathrm{L}, 0.60 \mathrm{mmol})$ was treated with 3bromo- $N, N$-dimethylaniline $2 \mathbf{f}(0.100 \mathrm{~g}, 0.50 \mathrm{mmol})$ in the presence of $\mathrm{KF}$ (0.070 g, $1.20 \mathrm{mmol})$ and 18-crown-6 (0.317 g, $1.20 \mathrm{mmol})$ in THF (2.0 mL) under an atmosphere of $\mathrm{CO}_{2}$ (balloon pressure) at $-10^{\circ} \mathrm{C}$ to $\mathrm{rt}$ for $12 \mathrm{~h}$. Then the reaction mixture was kept stirring at $60{ }^{\circ} \mathrm{C}$ for $12 \mathrm{~h}$ followed by flash column 
chromatography (Pet. ether $/ \mathrm{EtOAc}=98 / 02$ ) of the crude reaction mixture using silica gel to afford methyl 2-((3-bromophenyl)(methyl)amino)benzoate 3f as a yellow viscous oil (0.138 g, $86 \%$ yield).

$\boldsymbol{R}_{\mathbf{f}}\left(\right.$ Pet. ether $/$ EtOAc $=$ 95/05): 0.45; ${ }^{1} \mathbf{H}$ NMR $\left(\mathbf{4 0 0} \mathbf{~ M H z}, \mathbf{C D C l}_{\mathbf{3}}\right) \delta 7.90(\mathrm{~d}, J=7.7 \mathrm{~Hz}, 1 \mathrm{H})$, 7.58 (t, $J=7.6 \mathrm{~Hz}, 1 \mathrm{H}), 7.35$ (t, $J=7.6 \mathrm{~Hz}, 1 \mathrm{H}), 7.29$ (d, $J=8.0 \mathrm{~Hz}, 1 \mathrm{H}), 7.00$ (t, $J=8.0 \mathrm{~Hz}$, $1 \mathrm{H}), 6.85$ (d, $J=7.8 \mathrm{~Hz}, 1 \mathrm{H}), 6.76(\mathrm{~s}, 1 \mathrm{H}), 6.48$ (d, $J=8.2 \mathrm{~Hz}, 1 \mathrm{H}), 3.69$ (s, 3H), 3.27 (s, 3H). ${ }^{13}$ C NMR (100 MHz, $\left.\mathbf{C D C l}_{3}\right) \delta 166.96,150.48,147.25,133.72,131.85,130.16,129.90,129.51$, 126.38, 123.19, 120.34, 116.15, 112.26, 52.30, 40.30. HRMS (ESI) calculated $[\mathrm{M}+\mathrm{H}]^{+}$for $\mathrm{C}_{15} \mathrm{H}_{15} \mathrm{O}_{2} \mathrm{NBr}$ : 320.0281, found: 320.0284. FTIR ( $\left.\mathbf{c m}^{-1}\right)$ : 3020, 2953, 2402, 1722, 1590, 1485, $1348,1253,1129,1080,760$.

\section{Dimethyl 2,2'-(methylazanediyl)dibenzoate (3g)}<smiles>COC(=O)c1ccccc1N(c1ccccc1)c1ccccc1C(=O)OC</smiles>

Following the general procedure, 2-(trimethylsilyl)phenyl trifluoromethanesulfonate 1a $(0.179 \mathrm{~g}, 146 \mu \mathrm{L}, 0.60 \mathrm{mmol})$ was treated with methyl 2-(dimethylamino)benzoate $2 \mathrm{~g}(0.090 \mathrm{~g}, 0.50 \mathrm{mmol})$ in the presence of KF $(0.070 \mathrm{~g}, 1.20 \mathrm{mmol})$ and 18-crown-6 (0.317 g, 1.20 $\mathrm{mmol})$ in THF $(2.0 \mathrm{~mL})$ under an atmosphere of $\mathrm{CO}_{2}$ (balloon pressure) at $-10{ }^{\circ} \mathrm{C}$ to $\mathrm{rt}$ for $12 \mathrm{~h}$. Then the reaction mixture was kept stirring at $60{ }^{\circ} \mathrm{C}$ for $12 \mathrm{~h}$ followed by flash column chromatography (Pet. ether /EtOAc $=90 / 10)$ of the crude reaction mixture using silica gel to afford dimethyl 2,2'-(methylazanediyl)dibenzoate $\mathbf{3 g}$ as a yellow viscous oil ( $0.096 \mathrm{~g}, 64 \%$ yield $)$.

$\boldsymbol{R}_{\mathbf{f}}($ Pet. ether $/$ EtOAc $=90 / 10): 0.34 ;{ }^{1} \mathbf{H}$ NMR $\left(\mathbf{4 0 0} \mathbf{~ M H z}, \mathbf{C D C l}_{3}\right) \delta 7.56(\mathrm{~d}, J=6.6 \mathrm{~Hz}, 2 \mathrm{H})$, $7.41(\mathrm{t}, J=7.2 \mathrm{~Hz}, 2 \mathrm{H}), 7.13(\mathrm{~d}, J=8.2 \mathrm{~Hz}, 2 \mathrm{H}), 7.00(\mathrm{t}, J=7.5 \mathrm{~Hz}, 2 \mathrm{H}), 3.47(\mathrm{~s}, 6 \mathrm{H}), 3.37$ (s, 3H). ${ }^{13} \mathbf{C}$ NMR (100 MHz, $\left.\mathbf{C D C l}_{3}\right) \delta$ 167.97, 149.18, 132.42, 131.17, 124.77, 123.23, 122.01, 51.75, 42.83. HRMS (ESI) calculated $[\mathrm{M}+\mathrm{H}]^{+}$for $\mathrm{C}_{17} \mathrm{H}_{18} \mathrm{O}_{4} \mathrm{~N}$ : 300.1230, found: 300.1228 . FTIR $\left(\mathbf{c m}^{-1}\right)$ : 3020, 1726, 1596, 1484, 1450, 1291, 1240, 1129, 1080, 767.

\section{Methyl 2-((3,4-difluorophenyl)(methyl)amino)benzoate (3h)}

Following the general procedure, 2-(trimethylsilyl)phenyl trifluoromethanesulfonate 1a $(0.179 \mathrm{~g}$, $146 \mu \mathrm{L}, 0.60 \mathrm{mmol})$ was treated with 3,4-difluoro- $N, N$-dimethylaniline $2 \mathbf{h}(0.078 \mathrm{~g}, 0.50 \mathrm{mmol})$ in the presence of KF $(0.070 \mathrm{~g}, 1.20 \mathrm{mmol})$ and 18 -crown-6 $(0.317 \mathrm{~g}, 1.20 \mathrm{mmol})$ in THF $(2.0$ 


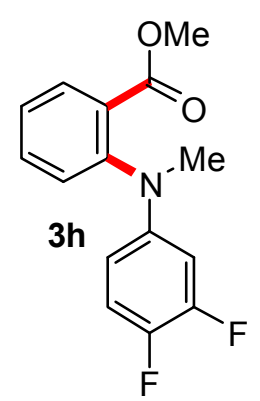

$\mathrm{mL}$ ) under an atmosphere of $\mathrm{CO}_{2}$ (balloon pressure) at $-10{ }^{\circ} \mathrm{C}$ to $\mathrm{rt}$ for $12 \mathrm{~h}$. Then the reaction mixture was kept stirring at $60{ }^{\circ} \mathrm{C}$ for $12 \mathrm{~h}$ followed by flash column chromatography $($ Pet. ether $/$ EtOAc $=98 / 02)$ of the crude reaction mixture using silica gel to afford methyl 2-((3,4difluorophenyl)(methyl)amino)benzoate $\mathbf{3 h}$ as a yellow viscous oil $(0.103 \mathrm{~g}$, $74 \%$ yield).

$\boldsymbol{R}_{\mathbf{f}}($ Pet. ether $/$ EtOAc $=95 / 05): 0.45 ;{ }^{1} \mathbf{H}$ NMR $\left(400 \mathbf{~ M H z}, \mathbf{C D C l}_{3}\right) \delta 7.84(\mathrm{~d}, J=7.5 \mathrm{~Hz}, 1 \mathrm{H})$, $7.55(\mathrm{t}, J=7.3 \mathrm{~Hz}, 1 \mathrm{H}), 7.31$ (t, $J=7.6 \mathrm{~Hz}, 1 \mathrm{H}), 7.26$ (d, $J=7.8 \mathrm{~Hz}, 1 \mathrm{H}), 6.91$ (dd, $J_{l}=19.0 \mathrm{~Hz}$, $\left.J_{2}=9.3 \mathrm{~Hz}, 1 \mathrm{H}\right), 6.39-6.33(\mathrm{~m}, 1 \mathrm{H}), 6.22-6.20(\mathrm{~m}, 1 \mathrm{H}), 3.66(\mathrm{~s}, 3 \mathrm{H}), 3.22(\mathrm{~s}, 3 \mathrm{H}) .{ }^{13} \mathbf{C}$ NMR $\left(100 \mathrm{MHz}, \mathbf{C D C l}_{3}\right) \delta 167.03,150.62\left(\mathrm{dd}, J_{1}=244.4 \mathrm{~Hz}, J_{2}=12.8 \mathrm{~Hz}\right), 146.54(\mathrm{~d}, J=8.2 \mathrm{~Hz})$, $143.24\left(\mathrm{dd}, J_{1}=237.7 \mathrm{~Hz}, J_{2}=13.2 \mathrm{~Hz}\right), 133.75,131.87,129.68,129.46,126.28,117.14(\mathrm{~d}, J=$ $17.8 \mathrm{~Hz}), 108.85\left(\mathrm{dd}, J_{1}=5.2 \mathrm{~Hz}, J_{2}=3.0 \mathrm{~Hz}\right), 52.31,40.63$. HRMS (ESI) calculated [M+H] ${ }^{+}$ for $\mathrm{C}_{15} \mathrm{H}_{14} \mathrm{O}_{2} \mathrm{NF}_{2}$ : 278.0987, found: 278.0986. FTIR $\left(\mathbf{c m}^{-1}\right)$ : 3022, 3951, 2256, 1722, 1598, $1517,1445,1363,1289,1082,951,909,757$.

\section{Methyl 2-((3,5-dimethylphenyl)(methyl)amino)benzoate (3i)}

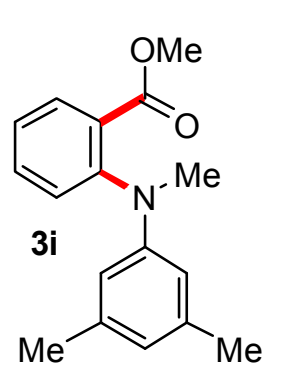

Following the general procedure, 2-(trimethylsilyl)phenyl trifluoromethanesulfonate 1a $(0.179 \mathrm{~g}, 146 \mu \mathrm{L}, 0.60 \mathrm{mmol})$ was treated with $N, N, 3,5$-tetramethylaniline $2 \mathbf{i}(0.075 \mathrm{~g}, 82 \mu \mathrm{L}, 0.50 \mathrm{mmol})$ in the presence of $\mathrm{KF}(0.070 \mathrm{~g}, 1.20 \mathrm{mmol})$ and 18-crown-6 $(0.317 \mathrm{~g}, 1.20 \mathrm{mmol})$ in THF (2.0 $\mathrm{mL}$ ) under an atmosphere of $\mathrm{CO}_{2}$ (balloon pressure) at $-10{ }^{\circ} \mathrm{C}$ to $\mathrm{rt}$ for $12 \mathrm{~h}$. Then the reaction mixture was kept stirring at $60^{\circ} \mathrm{C}$ for $12 \mathrm{~h}$ followed by flash column chromatography (Pet. ether $/$ EtOAc $=98 / 02)$ of the crude reaction mixture using silica gel to afford methyl 2-((3,5-dimethylphenyl)(methyl)amino)benzoate $\mathbf{3 i}$ as a yellow viscous oil $(0.117 \mathrm{~g}, 87 \%$ yield $)$.

$\boldsymbol{R}_{\mathbf{f}}($ Pet. ether $/$ EtOAc $=95 / 05): 0.50 ;{ }^{1} \mathbf{H}$ NMR $\left(\mathbf{5 0 0} \mathbf{~ M H z}, \mathbf{C D C l}_{\mathbf{3}}\right) \delta 7.87\left(\mathrm{dd}, J_{l}=7.7 \mathrm{~Hz}, J_{2}=\right.$ $1.5 \mathrm{~Hz}, 1 \mathrm{H}), 7.57$ (td, $\left.J_{1}=7.9 \mathrm{~Hz}, J_{2}=1.6 \mathrm{~Hz}, 1 \mathrm{H}\right), 7.35-7.29(\mathrm{~m}, 2 \mathrm{H}), 6.50(\mathrm{~s}, 1 \mathrm{H}), 6.37(\mathrm{~s}, 2 \mathrm{H})$, 3.69 (s, 3H), 3.34 (s, 3H), 2.29 (s, 6H). ${ }^{13} \mathbf{C}$ NMR (125 MHz, $\left.\mathbf{C D C l}_{3}\right) \delta$ 167.56, 149.26, 148.34, $138.38,133.10,131.30,129.15,128.82,124.91,120.18,112.48,52.00,40.50,21.65$. HRMS (ESI) calculated $[\mathrm{M}+\mathrm{H}]^{+}$for $\mathrm{C}_{17} \mathrm{H}_{20} \mathrm{O}_{2} \mathrm{~N}: 270.1489$, found: 270.1489 . FTIR $\left(\mathbf{c m}^{-1}\right)$ : 3017, 1722 , 1596, 1485, 1351, 1297, 1253, 1215, 759 . 


\section{Methyl 2-(methyl(naphthalen-1-yl)amino)benzoate (3j)}

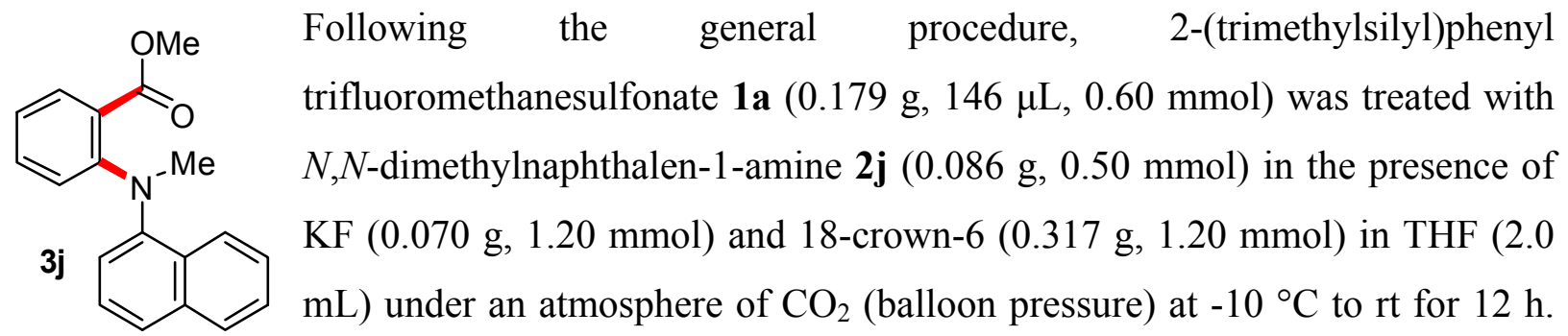

Then the reaction mixture was kept stirring at $60{ }^{\circ} \mathrm{C}$ for $12 \mathrm{~h}$ followed by flash column chromatography (Pet. ether $/$ EtOAc $=98 / 02)$ of the crude reaction mixture using silica gel to afford methyl 2-(methyl(naphthalen-1-yl)amino)benzoate $\mathbf{3 j}$ as a yellow solid (0.132 $\mathrm{g}$, 90\% yield).

$\boldsymbol{R}_{\mathbf{f}}($ Pet. ether $/$ EtOAc $=95 / 05): 0.46 ;{ }^{1} \mathbf{H}$ NMR (400 MHz, $\left.\mathbf{C D C l}_{3}\right) \delta 8.25(\mathrm{~d}, J=7.6 \mathrm{~Hz}, 1 \mathrm{H})$, $7.88(\mathrm{~d}, J=7.6 \mathrm{~Hz}, 1 \mathrm{H}), 7.68(\mathrm{~d}, J=8.3 \mathrm{~Hz}, 1 \mathrm{H}), 7.58-7.50(\mathrm{~m}, 3 \mathrm{H}), 7.43-7.37$ (m, 2H), 7.15 (d, $J=7.3 \mathrm{~Hz}, 1 \mathrm{H}), 7.10(\mathrm{~d}, J=8.3 \mathrm{~Hz}, 1 \mathrm{H}), 6.99(\mathrm{t}, J=7.4 \mathrm{~Hz}, 1 \mathrm{H}), 3.40(\mathrm{~s}, 3 \mathrm{H}), 3.01(\mathrm{~s}, 3 \mathrm{H}) .{ }^{13} \mathbf{C}$ NMR (100 MHz, $\left.\mathbf{C D C l}_{3}\right) \delta$ 169.10, 149.57, 147.64, 135.00, 131.98, 130.95, 129.96, 128.34, $126.11,126.01,125.89,125.36,124.26,123.41,121.60,120.01,118.36,51.26,42.42$. HRMS (ESI) calculated $[\mathrm{M}+\mathrm{H}]^{+}$for $\mathrm{C}_{19} \mathrm{H}_{18} \mathrm{O}_{2} \mathrm{~N}: 292.1332$, found: 292.1331. FTIR (cm $\left.{ }^{-1}\right): 3017,1715$, $1595,1482,1445,1320,1288,761$.

\section{Methyl (E)-2-((4-(2-(5-bromothiophen-2-yl)vinyl)phenyl)(methyl)amino)benzoate (3k)}

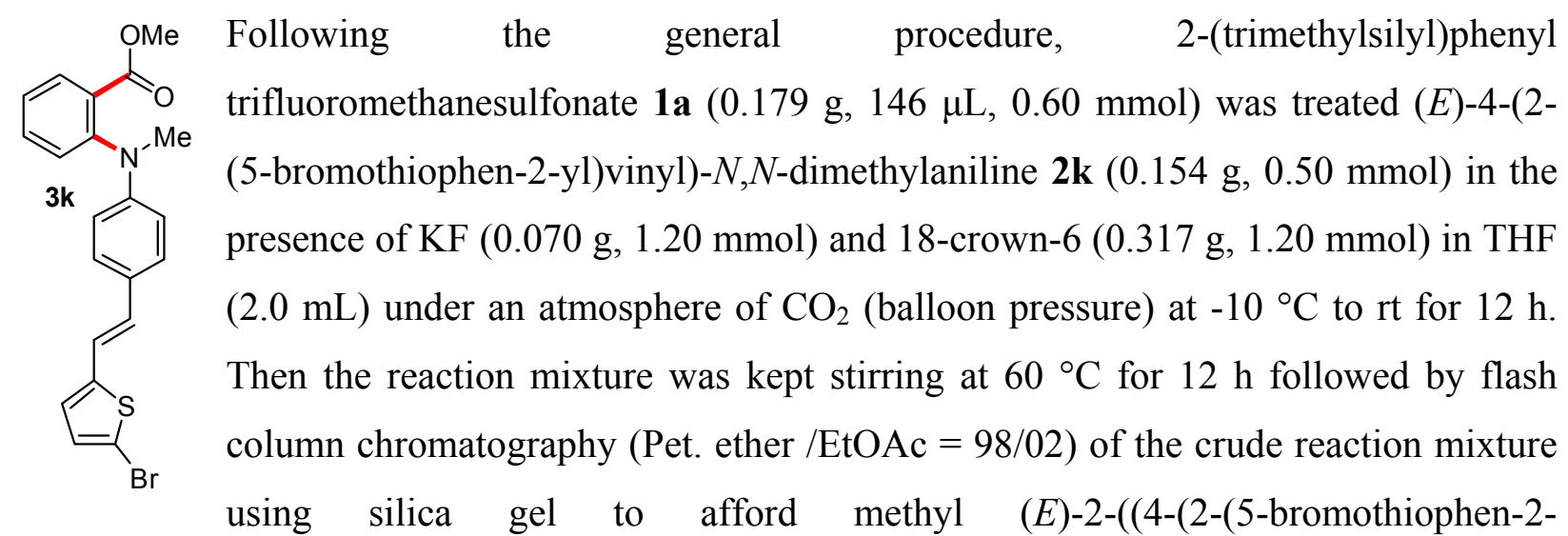
yl)vinyl)phenyl)(methyl)amino)benzoate 3k as a yellow solid (0.120 g, 56\% yield).

$\boldsymbol{R}_{\mathbf{f}}\left(\right.$ Pet. ether $/$ EtOAc $=$ 95/05): 0.43; ${ }^{1} \mathbf{H}$ NMR (400 $\left.\mathbf{M H z}, \mathbf{C D C l}_{3}\right) \delta 7.86(\mathrm{~d}, J=7.7 \mathrm{~Hz}, 1 \mathrm{H})$, $7.57(\mathrm{t}, J=7.5 \mathrm{~Hz}, 1 \mathrm{H}), 7.34-7.25(\mathrm{~m}, 4 \mathrm{H}), 6.92-6.87(\mathrm{~m}, 2 \mathrm{H}), 6.75-6.70(\mathrm{~m}, 2 \mathrm{H}), 6.58(\mathrm{~d}, J=$ $8.5 \mathrm{~Hz}, 2 \mathrm{H}), 3.63$ (s, 3H), 3.31 (s, 3H). ${ }^{13} \mathbf{C}$ NMR (100 MHz, $\left.\mathbf{C D C l}_{3}\right) \delta 167.22,149.14,147.64$, 
$145.58,133.58,131.72,130.44,129.61,129.48,129.13,127.39,126.23,125.97,124.90,117.52$, 113.88, 109.71, 52.29, 40.41. HRMS (ESI) calculated $[\mathrm{M}+\mathrm{H}]^{+}$for $\mathrm{C}_{21} \mathrm{H}_{19} \mathrm{O}_{2} \mathrm{NBrS}$ : 428.0314, found: 428.0308. FTIR (cm $\left.{ }^{-1}\right)$ : 3031, 2982, 2360, 2338, 1733, 1647, 1602, 1582, 1454, 1271, $1190,1027,950,757$.

Dimethyl 2,2'-(((phenylmethylene)bis(4,1-phenylene))bis(methylazanediyl))dibenzoate (3I)

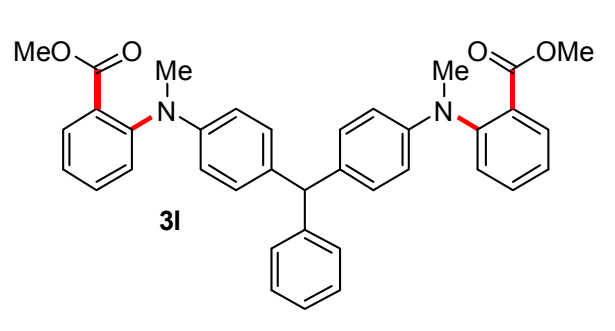

Following the general procedure, 2-(trimethylsilyl)phenyl trifluoromethanesulfonate $1 \mathrm{a}(0.179 \mathrm{~g}, 146 \mu \mathrm{L}, 0.60$ mmol) was treated with 4,4'-(phenylmethylene)bis $(N, N$ dimethylaniline) 21 (0.083 g, $0.25 \mathrm{mmol})$ in the presence of $\mathrm{KF}(0.070 \mathrm{~g}, 1.20 \mathrm{mmol})$ and 18-crown-6 (0.317 g, 1.20 mmol) in THF (2.0 mL) under an atmosphere of $\mathrm{CO}_{2}$ (balloon pressure) at $-10^{\circ} \mathrm{C}$ to $\mathrm{rt}$ for $12 \mathrm{~h}$. Then the reaction mixture was kept stirring at $100{ }^{\circ} \mathrm{C}$ for $12 \mathrm{~h}$ followed by flash column chromatography $($ Pet. ether $/$ EtOAc $=90 / 10)$ of the crude reaction mixture using silica gel to afford dimethyl 2,2'-(((phenylmethylene)bis(4,1-phenylene))bis(methylazanediyl))dibenzoate 31 as a yellow viscous oil $(0.120 \mathrm{~g}, 84 \%$ yield $)$.

$\boldsymbol{R}_{\mathbf{f}}($ Pet. ether $/$ EtOAc $=90 / 10): 0.40 ;{ }^{1} \mathbf{H}$ NMR (400 MHz, $\left.\mathbf{C D C l}_{\mathbf{3}}\right) \delta 7.80(\mathrm{~d}, J=7.7 \mathrm{~Hz}, 2 \mathrm{H})$, $7.53(\mathrm{t}, J=7.4 \mathrm{~Hz}, 2 \mathrm{H}), 7.34-7.29(\mathrm{~m}, 3 \mathrm{H}), 7.27-7.25(\mathrm{~m}, 2 \mathrm{H}), 7.23-7.15(\mathrm{~m}, 4 \mathrm{H}), 6.96(\mathrm{~d}, J=$ $8.4 \mathrm{~Hz}, 4 \mathrm{H}), 6.63(\mathrm{~d}, J=8.4 \mathrm{~Hz}, 4 \mathrm{H}), 5.39(\mathrm{~s}, 1 \mathrm{H}), 3.63(\mathrm{~s}, 6 \mathrm{H}), 3.32(\mathrm{~s}, 6 \mathrm{H}) .{ }^{13} \mathbf{C}$ NMR (100 MHz, $\left.\mathbf{C D C l}_{3}\right) \delta 167.60,148.39,147.59,145.17,134.27,133.14,131.32,129.81,129.35,128.96$, 128.55, 128.10, 125.91, 124.84, 114.55, 55.21, 52.06, 40.59. HRMS (ESI) calculated [M+H] ${ }^{+}$ for $\mathrm{C}_{37} \mathrm{H}_{35} \mathrm{O}_{4} \mathrm{~N}_{2}$ : 571.2591, found: 571.2592. FTIR (cm $\left.{ }^{-1}\right)$ : 3022, 1724, 1597, 1512, 1489, 1451, 1347, 1296, 1248, 1217, 1129, 1091, 767.

Ethyl 2-(ethyl(phenyl)amino)benzoate (3m)

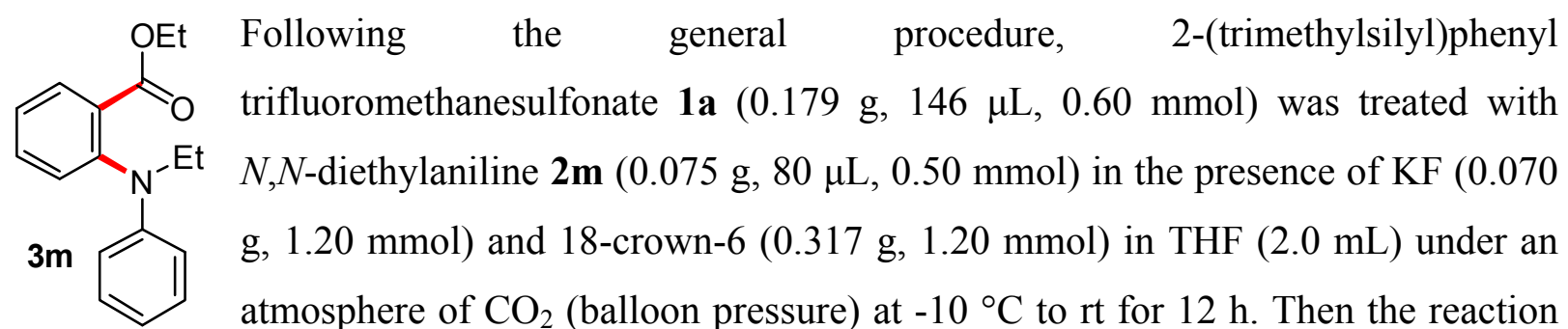

mixture was kept stirring at $60{ }^{\circ} \mathrm{C}$ for $12 \mathrm{~h}$ followed by flash column chromatography (Pet. ether 
/EtOAc $=98 / 02)$ of the crude reaction mixture using silica gel to afford ethyl 2(ethyl(phenyl)amino)benzoate $\mathbf{3 m}$ as a yellow viscous oil $(0.127 \mathrm{~g}, 94 \%$ yield).

$\boldsymbol{R}_{\mathbf{f}}($ Pet. ether $/$ EtOAc $=95 / 05): 0.60 ;{ }^{1} \mathbf{H}$ NMR (400 MHz, $\left.\mathbf{C D C l}_{3}\right) \delta 7.84(\mathrm{~d}, J=7.2 \mathrm{~Hz}, 1 \mathrm{H})$, $7.55(\mathrm{t}, J=7.6 \mathrm{~Hz}, 1 \mathrm{H}), 7.32-7.28(\mathrm{~m}, 2 \mathrm{H}), 7.16(\mathrm{t}, J=7.8 \mathrm{~Hz}, 2 \mathrm{H}), 6.71(\mathrm{t}, J=7.2 \mathrm{~Hz}, 1 \mathrm{H})$, $6.62(\mathrm{~d}, J=8.2 \mathrm{~Hz}, 2 \mathrm{H}), 4.06(\mathrm{q}, J=7.1 \mathrm{~Hz}, 2 \mathrm{H}), 3.76(\mathrm{q}, J=7.1 \mathrm{~Hz}, 2 \mathrm{H}), 1.27$ (t, $J=7.1 \mathrm{~Hz}$, $3 \mathrm{H}), 1.11$ (t, $J=7.1 \mathrm{~Hz}, 3 \mathrm{H}) .{ }^{13} \mathbf{C}$ NMR (100 MHz, $\left.\mathbf{C D C l}_{3}\right) \delta 167.28,148.48,146.31,133.03$, $131.49,130.76,130.03,128.97,125.54,117.46,114.16,61.04,46.97,14.03$, 13.14. HRMS (ESI) calculated $[\mathrm{M}+\mathrm{H}]^{+}$for $\mathrm{C}_{17} \mathrm{H}_{20} \mathrm{O}_{2} \mathrm{~N}: 270.1489$, found: 270.1484 . FTIR (cm $\left.{ }^{-1}\right): 3019,1714$, 1594, 1492, 1376, 1289, 1223, 760.

\section{Benzyl 2-(methyl(phenyl)amino)benzoate (3n)}

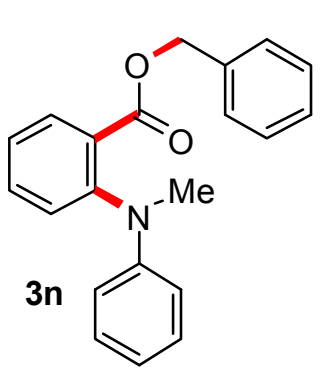

Following the general procedure, 2-(trimethylsilyl)phenyl

trifluoromethanesulfonate $1 \mathrm{a}(0.179 \mathrm{~g}, 146 \mu \mathrm{L}, 0.60 \mathrm{mmol})$ was treated with $N$-benzyl- $N$-methylaniline $2 \mathrm{n}(0.099 \mathrm{~g}, 0.50 \mathrm{mmol})$ in the presence of $\mathrm{KF}(0.070 \mathrm{~g}, 1.20 \mathrm{mmol})$ and 18-crown-6 (0.317 $\mathrm{g}, 1.20 \mathrm{mmol})$ in THF $(2.0 \mathrm{~mL})$ under an atmosphere of $\mathrm{CO}_{2}$ (balloon pressure) at $-10{ }^{\circ} \mathrm{C}$ to $\mathrm{rt}$ for $12 \mathrm{~h}$. Then the reaction mixture was kept stirring at $60{ }^{\circ} \mathrm{C}$ for $12 \mathrm{~h}$ followed by flash column chromatography (Pet. ether $/$ EtOAc $=98 / 02$ ) of the crude reaction mixture using silica gel to afford benzyl 2-(methyl(phenyl)amino)benzoate 3n as a yellow viscous oil ( $0.130 \mathrm{~g}, 82 \%$ yield $)$.

$\boldsymbol{R}_{\mathbf{f}}\left(\right.$ Pet. ether $/$ EtOAc $=$ 95/05): 0.54; ${ }^{1} \mathbf{H}$ NMR (400 $\left.\mathbf{M H z}, \mathbf{C D C l}_{3}\right) \delta 7.93(\mathrm{~d}, J=7.7 \mathrm{~Hz}, 1 \mathrm{H})$, $7.58(\mathrm{t}, J=7.7 \mathrm{~Hz}, 1 \mathrm{H}), 7.37-7.33(\mathrm{~m}, 5 \mathrm{H}), 7.31-7.28(\mathrm{~m}, 2 \mathrm{H}), 7.25-7.21(\mathrm{~m}, 2 \mathrm{H}), 6.83(\mathrm{t}, J=$ $7.2 \mathrm{~Hz}, 1 \mathrm{H}), 6.67(\mathrm{~d}, J=8.2 \mathrm{~Hz}, 2 \mathrm{H}), 5.12(\mathrm{~s}, 2 \mathrm{H}), 3.27(\mathrm{~s}, 3 \mathrm{H}) .{ }^{13} \mathbf{C}$ NMR (100 MHz, CDCl$) \delta$ $166.85,149.14,148.07,135.73,133.40,131.54,129.76,129.41,128.94,128.47,128.33,128.12$, 125.50, 117.82, 114.00, 66.85, 40.29. HRMS (ESI) calculated $[\mathrm{M}+\mathrm{H}]^{+}$for $\mathrm{C}_{21} \mathrm{H}_{20} \mathrm{O}_{2} \mathrm{~N}$ : 318.1489, found: 318.1488. FTIR (cm $\left.{ }^{-1}\right): 3025,1721,1594,1493,1453,1345,1286,1247$, $1128,1077,755$.

\section{Methyl 4,5-dimethyl-2-(methyl(phenyl)amino)benzoate (3o)}

Following the general procedure, 4,5-dimethyl-2-(trimethylsilyl)phenyl trifluoromethanesulfonate $\mathbf{1 b}(0.196 \mathrm{~g}, 0.60 \mathrm{mmol})$ was treated with $N, N$-dimethylaniline $\mathbf{2 a}$ 


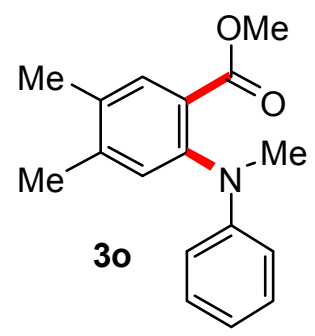

$(0.061 \mathrm{~g}, 64 \mu \mathrm{L}, 0.50 \mathrm{mmol})$ in the presence of $\mathrm{KF}(0.070 \mathrm{~g}, 1.20 \mathrm{mmol})$ and 18-crown-6 (0.317 g, $1.20 \mathrm{mmol})$ in THF $(2.0 \mathrm{~mL})$ under an atmosphere of $\mathrm{CO}_{2}$ (balloon pressure) at $-10{ }^{\circ} \mathrm{C}$ to $\mathrm{rt}$ for $12 \mathrm{~h}$. Then the reaction mixture was kept stirring at $60{ }^{\circ} \mathrm{C}$ for $12 \mathrm{~h}$ followed by flash column chromatography (Pet. ether $/$ EtOAc $=98 / 02)$ of the crude reaction mixture using silica gel to afford methyl 4,5-dimethyl-2(methyl(phenyl)amino)benzoate 30 as a yellow viscous oil $(0.119 \mathrm{~g}, 88 \%$ yield).

$\boldsymbol{R}_{\mathbf{f}}\left(\right.$ Pet. ether $/$ EtOAc $=$ 95/05): 0.50; ${ }^{1} \mathbf{H}$ NMR $\left(400 \mathbf{~ M H z}, \mathbf{C D C l}_{3}\right) \delta 7.67(\mathrm{~s}, 1 \mathrm{H}), 7.16(\mathrm{t}, J=$ $7.9 \mathrm{~Hz}, 2 \mathrm{H}), 7.08(\mathrm{~s}, 1 \mathrm{H}), 6.71(\mathrm{t}, J=7.2 \mathrm{~Hz}, 1 \mathrm{H}), 6.60(\mathrm{~d}, J=8.1 \mathrm{~Hz}, 2 \mathrm{H}), 3.62(\mathrm{~s}, 3 \mathrm{H}), 3.26$ (s, 3H), 2.32 (s, 3H), 2.30 (s, 3H). ${ }^{13} \mathbf{C}$ NMR (100 MHz, $\mathbf{C D C l}_{3}$ ) $\delta$ 167.34, 149.50, 145.91, 142.92, $134.42,132.62,130.88,128.88,126.59,117.23,113.46,52.00,40.24,19.93,19.23$. HRMS (ESI) calculated $[\mathrm{M}+\mathrm{H}]^{+}$for $\mathrm{C}_{17} \mathrm{H}_{20} \mathrm{O}_{2} \mathrm{~N}: 270.1489$, found: 270.1488. FTIR $\left(\mathbf{c m}^{-1}\right)$ : 2939, 1722, $1601,1497,1444,1339,1289,1251,1146,751$.

\section{Methyl 6-(methyl(phenyl)amino)benzo[d][1,3]dioxole-5-carboxylate (3p)}

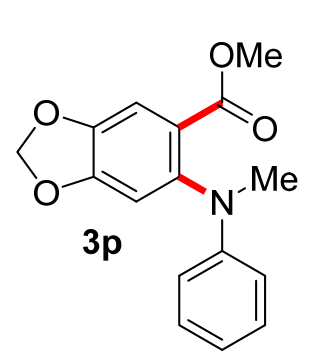

Following the general procedure, 6-(trimethylsilyl)benzo[ $d][1,3]$ dioxol-5-yl trifluoromethanesulfonate $1 \mathrm{c}(0.205 \mathrm{~g}, 0.60 \mathrm{mmol})$ was treated with $\mathrm{N}, \mathrm{N}$ dimethylaniline 2a $(0.061 \mathrm{~g}, 64 \mu \mathrm{L}, 0.50 \mathrm{mmol})$ in the presence of $\mathrm{KF}$ $(0.070 \mathrm{~g}, 1.20 \mathrm{mmol})$ and 18 -crown-6 $(0.317 \mathrm{~g}, 1.20 \mathrm{mmol})$ in THF $(2.0$ $\mathrm{mL}$ ) under an atmosphere of $\mathrm{CO}_{2}$ (balloon pressure) at $-10{ }^{\circ} \mathrm{C}$ to $\mathrm{rt}$ for $12 \mathrm{~h}$.

Then the reaction mixture was kept stirring at $60{ }^{\circ} \mathrm{C}$ for $12 \mathrm{~h}$ followed by flash column chromatography (Pet. ether $/$ EtOAc $=98 / 02$ ) of the crude reaction mixture using silica gel to afford methyl 6-(methyl(phenyl)amino)benzo[ $d][1,3]$ dioxole-5-carboxylate $\mathbf{3 p}$ as a yellow viscous oil ( $0.120 \mathrm{~g}, 84 \%$ yield $)$.

$\boldsymbol{R}_{\mathbf{f}}($ Pet. ether $/$ EtOAc $=95 / 05): 0.38 ;{ }^{1} \mathbf{H}$ NMR $\left(\mathbf{4 0 0} \mathbf{~ M H z}, \mathbf{C D C l}_{3}\right) \delta 7.35(\mathrm{~s}, 1 \mathrm{H}), 7.16(\mathrm{t}, J=7.8$ $\mathrm{Hz}, 2 \mathrm{H}), 6.73(\mathrm{~s}, 1 \mathrm{H}), 6.71(\mathrm{t}, J=7.2 \mathrm{~Hz}, 1 \mathrm{H}), 6.57(\mathrm{~d}, J=8.2 \mathrm{~Hz}, 2 \mathrm{H}), 6.05(\mathrm{~s}, 2 \mathrm{H}), 3.62$ (s, 3H), 3.21 (s, 3H). ${ }^{13} \mathbf{C}$ NMR (100 MHz, $\left.\mathbf{C D C l}_{3}\right) \delta$ 166.32, 151.73, 149.33, 145.69, 144.38, 128.96, 122.76, 117.31, 113.21, 110.67, 110.51, 102.29, 52.18, 40.11. HRMS (ESI) calculated $[\mathrm{M}+\mathrm{H}]^{+}$for $\mathrm{C}_{16} \mathrm{H}_{16} \mathrm{O}_{4} \mathrm{~N}$ : 286.1074, found: 286.1073. FTIR $\left(\mathbf{c m}^{-1}\right)$ : 3018, 2948, 1718, 1604, $1490,1440,1328,1251,1225,1129,1036,757$. 


\section{Methyl 4,5-difluoro-2-(methyl(phenyl)amino)benzoate (3q)}

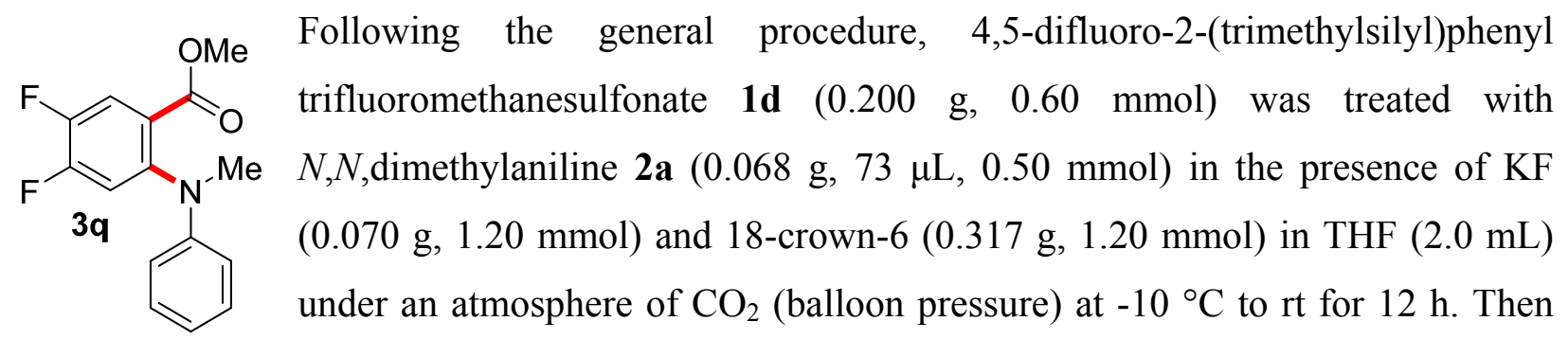

the reaction mixture was kept stirring at $60{ }^{\circ} \mathrm{C}$ for $12 \mathrm{~h}$ followed by flash column chromatography $($ Pet. ether $/$ EtOAc $=98 / 02)$ of the crude reaction mixture using silica gel to afford methyl 4,5-difluoro-2-(methyl(phenyl)amino)benzoate $\mathbf{3 q}$ as a yellow viscous oil (0.108 g, 78\% yield).

$\boldsymbol{R}_{\mathbf{f}}($ Pet. ether $/$ EtOAc $=95 / 05): 0.46 ;{ }^{1} \mathbf{H}$ NMR (400 MHz, $\left.\mathbf{C D C l}_{3}\right) \delta 7.67(\mathrm{t}, J=9.8 \mathrm{~Hz}, 1 \mathrm{H})$, $7.19(\mathrm{t}, J=7.8 \mathrm{~Hz}, 2 \mathrm{H}), 7.10\left(\mathrm{dd}, J_{1}=11.1 \mathrm{~Hz}, J_{2}=7.2 \mathrm{~Hz}, 1 \mathrm{H}\right), 6.79(\mathrm{t}, J=7.3 \mathrm{~Hz}, 1 \mathrm{H}), 6.65$ $(\mathrm{d}, J=8.1 \mathrm{~Hz}, 2 \mathrm{H}), 3.60$ (s, 3H), 3.26 (s, 3H). ${ }^{13} \mathbf{C}$ NMR (100 MHz, $\left.\mathbf{C D C l}_{3}\right) \delta 165.41,153.08$ $\left(\mathrm{dd}, J_{l}=257.1 \mathrm{~Hz}, J_{2}=13.6 \mathrm{~Hz}\right) 148.88,147.43\left(\mathrm{dd}, J_{1}=247.9 \mathrm{~Hz}, J_{2}=12.8 \mathrm{~Hz}\right), 145.70(\mathrm{~d}, J=$ 8.7 Hz), 129.18, 120.44 (d, $J=18.1 \mathrm{~Hz}), 118.98,117.96$ (d, $J=16.9 \mathrm{~Hz}), 114.80,52.45,40.57$. HRMS (ESI) calculated $[\mathrm{M}+\mathrm{H}]^{+}$for $\mathrm{C}_{15} \mathrm{H}_{14} \mathrm{O}_{2} \mathrm{NF}_{2}: 278.0987$, found: 278.0983. FTIR (cm ${ }^{-1}$ ): 3020, 2404, 1795, 1726, 1596, 1495, 1444, 1282, 1118, 930, 760.

\section{Methyl 3-(methyl(phenyl)amino)-2-naphthoate (3r)}

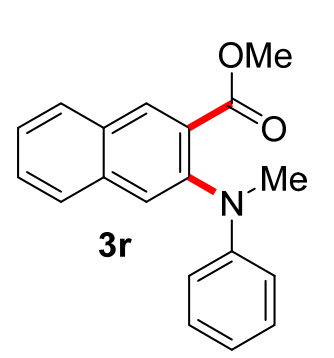

Following the general procedure, 3-(trimethylsilyl)naphthalen-2-yl trifluoromethanesulfonate $1 \mathrm{e}(0.209 \mathrm{~g}, 0.60 \mathrm{mmol})$ was treated with $N, N$ dimethylaniline $2 \mathrm{a}(0.061 \mathrm{~g}, 64 \mu \mathrm{L}, 0.50 \mathrm{mmol})$ in the presence of $\mathrm{KF}(0.070$ $\mathrm{g}, 1.20 \mathrm{mmol})$ and 18 -crown-6 $(0.317 \mathrm{~g}, 1.20 \mathrm{mmol})$ in THF $(2.0 \mathrm{~mL})$ under an atmosphere of $\mathrm{CO}_{2}$ (balloon pressure) at $-10{ }^{\circ} \mathrm{C}$ to $\mathrm{rt}$ for $12 \mathrm{~h}$. Then the

reaction mixture was kept stirring at $60{ }^{\circ} \mathrm{C}$ for $12 \mathrm{~h}$ followed by flash column chromatography (Pet. ether $/ \mathrm{EtOAc}=98 / 02)$ of the crude reaction mixture using silica gel to afford methyl 3(methyl(phenyl)amino)-2-naphthoate 3r as a yellow viscous oil (0.099 g, 68\% yield).

$\boldsymbol{R}_{\mathbf{f}}\left(\right.$ Pet. ether $/$ EtOAc = 95/05): 0.49; ${ }^{1} \mathbf{H}$ NMR (400 MHz, $\left.\mathbf{C D C l}_{3}\right) \delta 8.36(\mathrm{~s}, 1 \mathrm{H}), 7.90(\mathrm{~d}, J=$ $8.1 \mathrm{~Hz}, 1 \mathrm{H}), 7.79(\mathrm{~d}, J=8.1 \mathrm{~Hz}, 1 \mathrm{H}), 7.72(\mathrm{~s}, 1 \mathrm{H}), 7.56(\mathrm{t}, J=7.3 \mathrm{~Hz}, 1 \mathrm{H}), 7.50(\mathrm{t}, J=7.3 \mathrm{~Hz}$, 1H), $7.17(\mathrm{t}, J=7.9 \mathrm{~Hz}, 2 \mathrm{H}), 6.76(\mathrm{t}, J=7.3 \mathrm{~Hz}, 1 \mathrm{H}), 6.67(\mathrm{~d}, J=8.1 \mathrm{~Hz}, 2 \mathrm{H}), 3.62(\mathrm{~s}, 3 \mathrm{H}), 3.41$ (s, 3H). ${ }^{13}$ C NMR (100 MHz, $\left.\mathbf{C D C l}_{3}\right) \delta 167.65,149.71,144.31,136.10,132.90,130.56,129.06$, 
128.79, 128.46, 128.27, 127.22, 126.62, 126.31, 118.23, 114.65, 52.31, 40.97. HRMS (ESI) calculated $[\mathrm{M}+\mathrm{H}]^{+}$for $\mathrm{C}_{19} \mathrm{H}_{18} \mathrm{O}_{2} \mathrm{~N}: 292.1332$, found: 292.1331. FTIR $\left(\mathbf{c m}^{-1}\right): 3019,2947,2403$, $1721,1593,1495,1449,1356,1274,1215,1036,760$.

\section{Methyl 2-(methyl(phenyl)amino)-1-naphthoate (3s)}

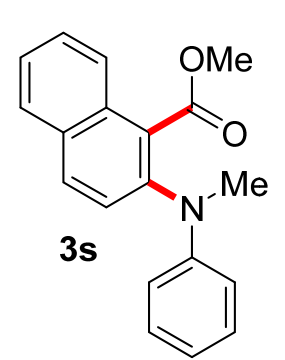

Following the general procedure, 1-(trimethylsilyl)naphthalen-2-yl trifluoromethanesulfonate 1f $(0.209 \mathrm{~g}, 0.60 \mathrm{mmol})$ was treated with $N, N$ dimethylaniline $2 \mathrm{a}(0.061 \mathrm{~g}, 64 \mu \mathrm{L}, 0.50 \mathrm{mmol})$ in the presence of $\mathrm{KF}(0.070 \mathrm{~g}$, $1.20 \mathrm{mmol})$ and 18 -crown-6 (0.317 g, $1.20 \mathrm{mmol})$ in THF $(2.0 \mathrm{~mL})$ under an atmosphere of $\mathrm{CO}_{2}$ (balloon pressure) at $-10{ }^{\circ} \mathrm{C}$ to $\mathrm{rt}$ for $12 \mathrm{~h}$. Then the reaction mixture was kept stirring at $60{ }^{\circ} \mathrm{C}$ for $12 \mathrm{~h}$ followed by flash column chromatography (Pet. ether $/$ EtOAc $=98 / 02)$ of the crude reaction mixture using silica gel to afford methyl 2(methyl(phenyl)amino)-1-naphthoate $3 \mathrm{~s}$ as a yellow viscous oil ( $0.090 \mathrm{~g}, 62 \%$ yield).

$\boldsymbol{R}_{\mathbf{f}}($ Pet. ether $/ \mathrm{EtOAc}=95 / 05): 0.49 ;{ }^{1} \mathbf{H} \mathbf{N M R}\left(\mathbf{4 0 0} \mathbf{~ M H z}, \mathbf{C D C l}_{3}\right) \delta 7.93(\mathrm{~d}, J=8.8 \mathrm{~Hz}, 1 \mathrm{H})$, $7.85(\mathrm{~d}, J=8.2 \mathrm{~Hz}, 2 \mathrm{H}), 7.56-7.49(\mathrm{~m}, 2 \mathrm{H}), 7.31$ (d, $J=8.8 \mathrm{~Hz}, 1 \mathrm{H}), 7.19(\mathrm{t}, J=7.8 \mathrm{~Hz}, 2 \mathrm{H})$, $6.80(\mathrm{t}, J=7.3 \mathrm{~Hz}, 1 \mathrm{H}), 6.73(\mathrm{~d}, J=8.2 \mathrm{~Hz}, 2 \mathrm{H}), 3.78(\mathrm{~s}, 3 \mathrm{H}), 3.31(\mathrm{~s}, 3 \mathrm{H}) .{ }^{13} \mathbf{C}$ NMR (100 MHz, $\left.\mathbf{C D C l}_{3}\right) \delta 168.95,149.19,144.68,131.90,131.58,131.10,129.36,128.92,128.23,127.48$, 126.43, 126.18, 124.77, 118.70, 115.35, 52.42, 40.50. HRMS (ESI) calculated $[\mathrm{M}+\mathrm{H}]^{+}$for $\mathrm{C}_{19} \mathrm{H}_{18} \mathrm{O}_{2} \mathrm{~N}:$ 292.1332, found: 292.1330. FTIR ( $\left.\mathbf{c m}^{-1}\right): 3020,2948,2814,2253,1724,1594$, 1506, 1447, 1357, 1282, 1128, 956, 760.

\section{Methyl 5-methyl-2-(methyl(phenyl)amino)benzoate and}

\section{Methyl 4-methyl-2-(methyl(phenyl)amino)benzoate (3t and 3t')}<smiles>COC(=O)c1ccc(C)cc1N(C)c1ccccc1N(C)c1ccccc1</smiles>

Following the general procedure, 4-methyl-2(trimethylsilyl)phenyl trifluoromethanesulfonate $\mathbf{1 g}$ $(0.187 \mathrm{~g}, \quad 0.60 \mathrm{mmol})$ was treated with $N, N$,dimethylaniline $2 \mathrm{a}(0.061 \mathrm{~g}, 64 \mu \mathrm{L}, 0.50 \mathrm{mmol})$ in the presence of KF $(0.070 \mathrm{~g}, 1.20 \mathrm{mmol})$ and 18 -crown-

$6(0.317 \mathrm{~g}, 1.20 \mathrm{mmol})$ in THF $(2.0 \mathrm{~mL})$ under an atmosphere of $\mathrm{CO}_{2}$ (balloon pressure) at -10 ${ }^{\circ} \mathrm{C}$ to $\mathrm{rt}$ for $12 \mathrm{~h}$. Then the reaction mixture was kept stirring at $60{ }^{\circ} \mathrm{C}$ for $12 \mathrm{~h}$ followed by flash column chromatography (Pet. ether $/ \mathrm{EtOAc}=98 / 02)$ of the crude reaction mixture using silica 
gel to afford methyl 5-methyl-2-(methyl(phenyl)amino)benzoate 3t and methyl 4-methyl-2(methyl(phenyl)amino)benzoate 3t' as a mixture of regioisomers in 1.4:1 ratio $(0.082 \mathrm{~g}, 64 \%$ yield, yellow viscous oil, regioisomer ratio determined by ${ }^{1} \mathrm{H}$ NMR analysis of crude reaction mixture ).

$\boldsymbol{R}_{\mathbf{f}}\left(\right.$ Pet. ether $/$ EtOAc $=$ 95/05): 0.41; ${ }^{1} \mathbf{H}$ NMR $\left(\mathbf{4 0 0} \mathbf{~ M H z}, \mathbf{C D C l}_{3}\right) ; \delta 7.75(\mathrm{~d}, J=7.7 \mathrm{~Hz}, 1 \mathrm{H})$, 7.19-7.14 (m, 2H), 7.10-7.08 (m, 2H), 6.75-6.70 (m, 1H), 6.63-6.59 (m, 2H), 3.60 (s, 3H), 3.27 (s, 3H), 2.38 (s, 3H). ${ }^{13} \mathbf{C}$ NMR (100 MHz, $\mathbf{C D C l}_{3}$ ); $\delta$ 167.39, 149.42, 148.29, 144.28, 134.17, $131.75,129.98,128.96,126.37,117.72,114.02,52.04,40.34,21.52$. Representative peaks of the minor isomer ${ }^{1} \mathbf{H}$ NMR (400 MHz, $\left.\mathbf{C D C l}_{3}\right) ; \delta 7.64(\mathrm{~s}, 1 \mathrm{H}), 7.35(\mathrm{~d}, J=7.1 \mathrm{~Hz}, 1 \mathrm{H}), 3.61$ (s, 3H), 3.26 (s, 3H), 2.40 (s, 3H). ${ }^{13} \mathbf{C}$ NMR (100 MHz, $\left.\mathbf{C D C l}_{3}\right) ; \delta$ 167.66, 149.51, 145.64, 135.57, 131.92, 129.56, 126.32, 117.53, 113.73, 52.16, 20.95. HRMS (ESI) calculated $[\mathrm{M}+\mathrm{H}]^{+}$ for $\mathrm{C}_{16} \mathrm{H}_{18} \mathrm{O}_{2} \mathrm{~N}$ : 256.1332, found: 256.1332. FTIR $\left(\mathrm{cm}^{-1}\right)$ : 3019, 2951, 1722, 1598, 1577, 1499 , 1344, 1297, 1249, 1064, 759 .

\section{Synthesis and Characterization of 2-Aminoaryl Benzoate Derivatives}

\section{4-(Ethoxycarbonyl)phenyl 2-(dimethylamino)benzoate (4a)}

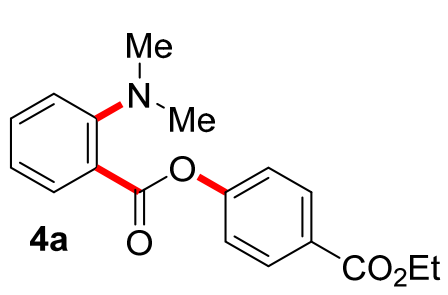

Following the general procedure, 2-(trimethylsilyl)phenyl trifluoromethanesulfonate 1a $(0.179 \mathrm{~g}, 146 \mu \mathrm{L}, 0.60 \mathrm{mmol})$ was treated with ethyl 4-(dimethylamino)benzoate $20(0.097 \mathrm{~g}, 0.50$ $\mathrm{mmol})$ in the presence of KF $(0.070 \mathrm{~g}, 1.20 \mathrm{mmol})$ and 18 -crown-6 $(0.317 \mathrm{~g}, 1.20 \mathrm{mmol})$ in THF $(2.0 \mathrm{~mL})$ under an atmosphere of $\mathrm{CO}_{2}$ (balloon pressure) at $-10{ }^{\circ} \mathrm{C}$ for $24 \mathrm{~h}$. Evaporation of solvent followed by flash column chromatography (Pet. ether $/$ EtOAc $=95 / 05$ ) of the crude reaction mixture using silica gel afforded 4-(ethoxycarbonyl)phenyl 2-(dimethylamino)benzoate 4a as a yellow viscous oil (0.117 g, $75 \%$ yield).

$\boldsymbol{R}_{\mathbf{f}}\left(\right.$ Pet. ether $/$ EtOAc $=$ 90/10): 0.38; ${ }^{1} \mathbf{H}$ NMR $\left(\mathbf{4 0 0} \mathbf{~ M H z}, \mathbf{C D C l}_{3}\right) \delta 8.12(\mathrm{~d}, J=8.6 \mathrm{~Hz}, 2 \mathrm{H})$, $7.95(\mathrm{~d}, J=7.8 \mathrm{~Hz}, 1 \mathrm{H}), 7.42(\mathrm{t}, J=7.8 \mathrm{~Hz}, 1 \mathrm{H}), 7.30(\mathrm{~d}, J=8.6 \mathrm{~Hz}, 2 \mathrm{H}), 7.00(\mathrm{~d}, J=8.4 \mathrm{~Hz}$, $1 \mathrm{H}), 6.89$ (t, $J=7.5 \mathrm{~Hz}, 1 \mathrm{H}), 4.38$ (q, $J=7.1 \mathrm{~Hz}, 2 \mathrm{H}), 2.92(\mathrm{~s}, 6 \mathrm{H}), 1.39(\mathrm{t}, J=7.1 \mathrm{~Hz}, 3 \mathrm{H}) .{ }^{13} \mathbf{C}$ NMR (100 MHz, $\left.\mathbf{C D C l}_{3}\right) \delta 165.91,165.48,154.81,153.40,133.39,132.41,131.13,127.82$, $121.72,118.55,118.49,116.98,61.05,43.82,14.35$. HRMS (ESI) calculated $[\mathrm{M}+\mathrm{H}]^{+}$for 
$\mathrm{C}_{18} \mathrm{H}_{20} \mathrm{O}_{4} \mathrm{~N}: 314.1387$, found: 314.1382. FTIR $\left(\mathbf{c m}^{-1}\right): 3017,1722,1600,1502,1444,1361$, $1278,1202,1163,1111,1025,758$.

\section{4-(Trifluoromethyl)phenyl 2-(dimethylamino)benzoate (4b)}

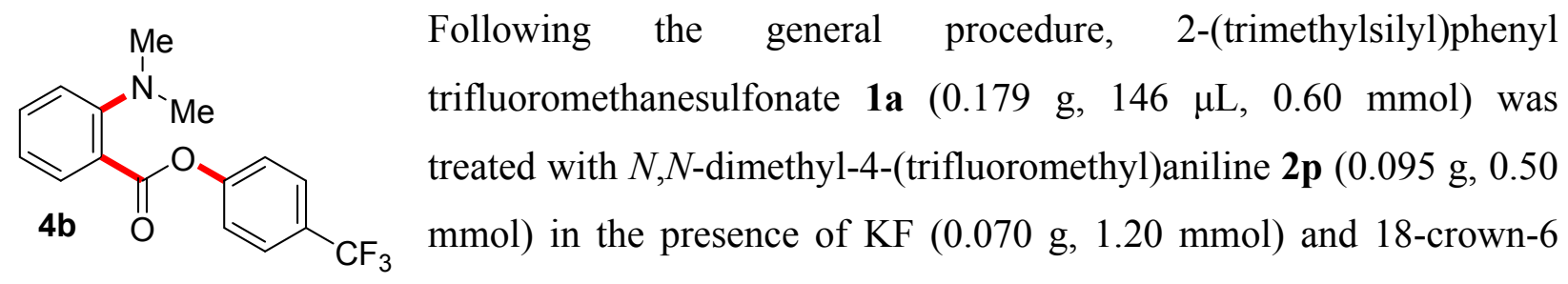

$(0.317 \mathrm{~g}, 1.20 \mathrm{mmol})$ in THF $(2.0 \mathrm{~mL})$ under an atmosphere of $\mathrm{CO}_{2}$ (balloon pressure) at $-10{ }^{\circ} \mathrm{C}$ to $\mathrm{rt}$ for $12 \mathrm{~h}$. Evaporation of solvent followed by flash column chromatography (Pet. ether /EtOAc $=98 / 02)$ of the crude reaction mixture using silica gel afforded 4(trifluoromethyl)phenyl 2-(dimethylamino)benzoate $4 \mathbf{b}$ as a yellow solid $(0.090 \mathrm{~g}, 58 \%$ yield). $\boldsymbol{R}_{\mathbf{f}}($ Pet. ether $/$ EtOAc $=95 / 05): 0.50 ;{ }^{1} \mathbf{H}$ NMR $\left(\mathbf{4 0 0} \mathbf{~ M H z}, \mathbf{C D C l}_{\mathbf{3}}\right) \delta 7.97(\mathrm{~d}, J=7.8 \mathrm{~Hz}, 1 \mathrm{H})$, $7.70(\mathrm{~d}, J=8.4 \mathrm{~Hz}, 2 \mathrm{H}), 7.46(\mathrm{t}, J=7.8 \mathrm{~Hz}, 1 \mathrm{H}), 7.36(\mathrm{~d}, J=8.4 \mathrm{~Hz}, 2 \mathrm{H}), 7.04$ (d, $J=8.4 \mathrm{~Hz}$, $1 \mathrm{H}), 6.92(\mathrm{t}, J=7.5 \mathrm{~Hz}, 1 \mathrm{H}), 2.95(\mathrm{~s}, 6 \mathrm{H}) .{ }^{13} \mathbf{C} \mathbf{~ N M R}\left(\mathbf{1 0 0} \mathbf{M H z}, \mathbf{C D C l}_{\mathbf{3}}\right) \delta 165.55,153.83$, 153.63, 133.63, 132.56, 127.98 (q, $J=32.8 \mathrm{~Hz}), 126.91$ (q, $J=3.5 \mathrm{~Hz}), 122.37,118.71,118.39$, 117.17, 43.96. HRMS (ESI) calculated $[\mathrm{M}+\mathrm{H}]^{+}$for $\mathrm{C}_{16} \mathrm{H}_{15} \mathrm{O}_{2} \mathrm{NF}_{3}: 310.1049$, found: 310.1049 . FTIR (cm $\left.{ }^{-1}\right):$ 2944, 1732, 1603, 1504, 1322, 1206, 1164, 1120, 1022, 758.

\section{$\underline{\text { X-ray Crystal Structure Analysis of } 4 \mathrm{~b}}$}

X-ray intensity data measurements of compound $\mathbf{4 b}$ was carried out on a Bruker D8 VENTURE Kappa Duo PHOTON II CPAD diffractometer equipped with Incoatech multilayer mirrors optics. The intensity measurements were carried out with micro-focus sealed tube diffraction source $\left(\mathrm{MoK}_{\alpha}=0.71073 \AA\right)$ at $100(2) \mathrm{K}$ temperature. The X-ray generator was operated at $50 \mathrm{kV}$ and $1.4 \mathrm{~mA}$. A preliminary set of cell constants and an orientation matrix were calculated from three sets of 36 frames. Data were collected with $\omega$ scan width of $0.5^{\circ}$ at different settings of $\varphi$ and $2 \theta$ with a frame time of 30 secs keeping the sample-to-detector distance fixed at $5.00 \mathrm{~cm}$. The X-ray data collection was monitored by APEX3 program (Bruker, 2016). ${ }^{4}$ All the data were corrected for Lorentzian, polarization and absorption effects using SAINT and SADABS programs (Bruker, 2016). SHELX-97 was used for structure

\footnotetext{
${ }^{4}$ Bruker (2016). APEX3, SAINT and SADABS. Bruker AXS Inc., Madison, Wisconsin, USA.
} 
solution and full matrix least-squares refinement on $F^{2} .5$ All the hydrogen atoms were located in difference Fourier and refined isotropically. The $\mathrm{CF}_{3}$ moiety of the structure showed rigid rotor type rotational disorder. Each $\mathrm{F}$ atom was anisotropically refined in three main positions with occupancies 50\% (for F1', F2' and F3'), 35\% (for F1, F2 and F3) and 15\% (for F1", F2" and F3"). An ORTEP III $^{6}$ view of compound was drawn with $50 \%$ probability displacement ellipsoids and $\mathrm{H}$ atoms are shown as small spheres of arbitrary radii. Only one orientation having major occupancy of $\mathrm{CF}_{3}$ moiety (i.e. F1', F2' and F3' with occupancy 50\%) is shown in the figure and the other positions of $\mathrm{CF}_{3}$ group are omitted for clarity.

Crystal data of $\mathbf{4 b ~} \mathrm{C}_{16} \mathrm{H}_{14} \mathrm{~F}_{3} \mathrm{NO}_{2}, \mathrm{M}=309.28$, colorless plate, $0.29 \times 0.09 \times 0.05 \mathrm{~mm}^{3}$, monoclinic, space group $P 2_{1} / c$, $a=8.0379(5) \AA, \quad b=$ $17.3908(11) \AA, c=10.6367(7)$ $\AA, \quad \beta=96.200(2)^{\circ}, \quad V=$ 1478.16(16) $\AA^{3}, Z=4, T=$ $100(2) \mathrm{K}, 2 \theta_{\max }=56.59^{\circ}, D_{\text {calc }}(\mathrm{g}$ $\left.\mathrm{cm}^{-3}\right)=1.390, F(000)=640, \mu$ $\left(\mathrm{mm}^{-1}\right)=0.117,24139$ reflections collected, 3672 unique reflections $\left(R_{\text {int }}=0.0285\right)$, 3285 observed $(I>2 \sigma(I))$ reflections, multi-scan absorption correction, $T_{\min }=$ 0.967, $T_{\max }=0.994,309$ refined

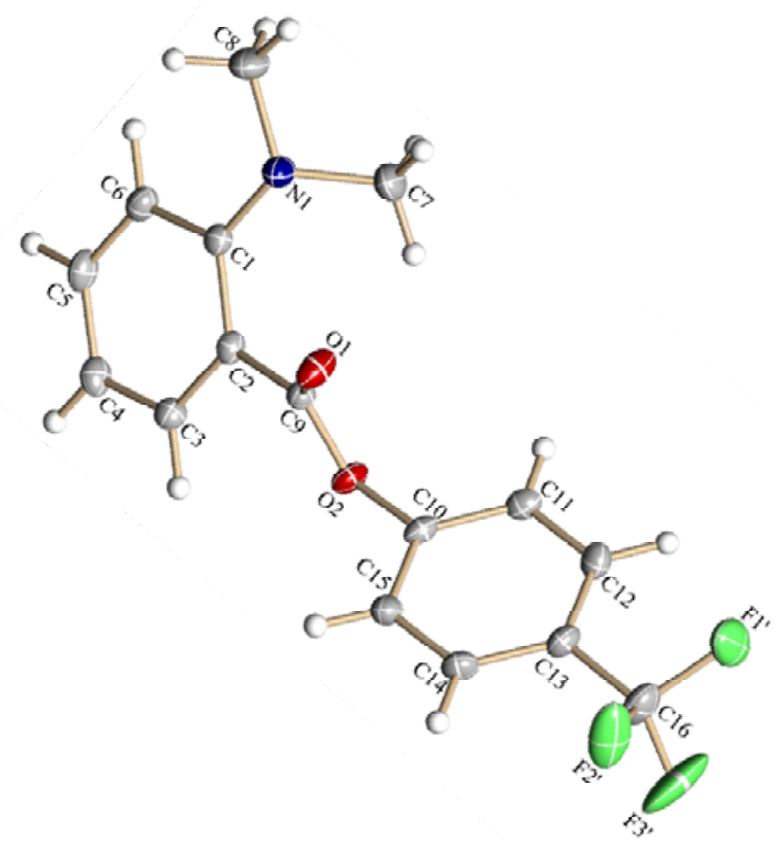
parameters, number of restraints $=61, S=1.084, R 1=0.0431, w R 2=0.1023$ (all data $R=$ $0.0495, w R 2=0.1061)$, maximum and minimum residual electron densities; $\Delta \rho_{\max }=0.39$, $\Delta \rho_{\min }=-0.19\left(\mathrm{e}^{-3}\right)$.

\footnotetext{
${ }^{5}$ Sheldrick, G. M. Acta Crystallogr. 2008, A64, 112.

${ }^{6}$ Farrugia, L. J. J. Appl. Cryst. 1997, 30, 565.
} 


\section{4-Cyanophenyl 2-(dimethylamino)benzoate (4c)}

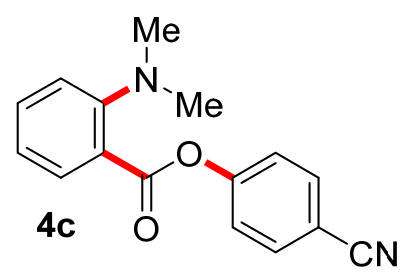

Following the general procedure, 2-(trimethylsilyl)phenyl trifluoromethanesulfonate $1 \mathrm{a}(0.179 \mathrm{~g}, 146 \mu \mathrm{L}, 0.60 \mathrm{mmol})$ was treated with 4-(dimethylamino)benzonitrile $2 \mathrm{q}(0.073 \mathrm{~g}, 0.50 \mathrm{mmol})$ in the presence of KF $(0.070 \mathrm{~g}, 1.20 \mathrm{mmol})$ and 18-crown-6 (0.317 $\mathrm{g}$, $1.20 \mathrm{mmol})$ in THF $(2.0 \mathrm{~mL})$ under an atmosphere of $\mathrm{CO}_{2}$ (balloon pressure) at $-10{ }^{\circ} \mathrm{C}$ to $\mathrm{rt}$ for $24 \mathrm{~h}$. Evaporation of solvent followed by flash column chromatography (Pet. ether $/$ EtOAc $=95 / 05)$ of the crude reaction mixture using silica gel afforded 4-cyanophenyl 2-(dimethylamino)benzoate $\mathbf{4 c}$ as a yellow viscous oil [The product is unstable in column chromatography. 61\% yield was determined by ${ }^{1} \mathrm{H}$ NMR analysis of crude reaction using $\mathrm{CH}_{2} \mathrm{Br}_{2}(36.0 \mu \mathrm{L}, 0.50 \mathrm{mmol})$ as the internal standard].

$\boldsymbol{R}_{\mathbf{f}}($ Pet. ether $/$ EtOAc $=80 / 20): 0.28 ;{ }^{1} \mathbf{H}$ NMR (400 MHz, $\left.\mathbf{C D C l}_{3}\right) \delta 7.98(\mathrm{~d}, J=7.8 \mathrm{~Hz}, 1 \mathrm{H})$, $7.73(\mathrm{~d}, J=8.2 \mathrm{~Hz}, 2 \mathrm{H}), 7.49$ (t, $J=7.2 \mathrm{~Hz}, 1 \mathrm{H}), 7.38$ (d, $J=8.2 \mathrm{~Hz}, 2 \mathrm{H}), 7.18-7.10(\mathrm{~m}, 1 \mathrm{H})$, 7.00-6.92 (m, 1H), 2.98 (s, 6H). ${ }^{13} \mathbf{C}$ NMR (100 MHz, $\left.\mathbf{C D C l}_{3}\right) \delta 164.89,154.57,134.04,133.83$, 132.67, 123.06, 118.49, 117.18, 116.54, 109.66, 44.32. HRMS (ESI) calculated $[\mathrm{M}+\mathrm{H}]^{+}$for $\mathrm{C}_{16} \mathrm{H}_{15} \mathrm{O}_{2} \mathrm{~N}_{2}$ : 267.1128, found: 267.1125. FTIR (cm $\left.{ }^{-1}\right): 3020,2225,1609,1490,1288,1216$, $1168,773$.

\section{(E)-4-(3-Ethoxy-3-oxoprop-1-en-1-yl)phenyl 2-(dimethylamino)benzoate (4d)}

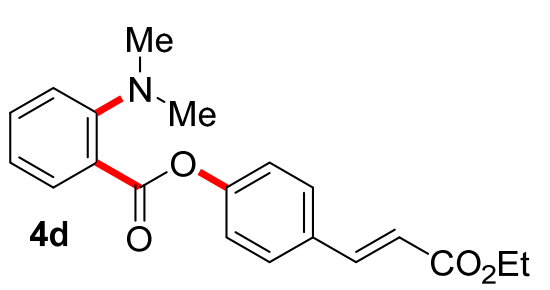

Following the general procedure, 2-(trimethylsilyl)phenyl trifluoromethanesulfonate 1a $(0.179 \mathrm{~g}, 146 \mu \mathrm{L}, 0.60 \mathrm{mmol})$ was treated with ethyl (E)-3-(4-(dimethylamino) phenyl)acrylate $2 \mathbf{r}(0.110 \mathrm{~g}, 0.50 \mathrm{mmol})$ in the presence of $\mathrm{KF}$ $(0.070 \mathrm{~g}, 1.20 \mathrm{mmol})$ and 18 -crown-6 $(0.317 \mathrm{~g}, 1.20 \mathrm{mmol})$ in

THF $\left(2.0 \mathrm{~mL}\right.$ ) under an atmosphere of $\mathrm{CO}_{2}$ (balloon pressure) at $-10{ }^{\circ} \mathrm{C}$ to $\mathrm{rt}$ for $12 \mathrm{~h}$. Evaporation of solvent followed by flash column chromatography (Pet. ether $/$ EtOAc $=93 / 07$ ) of the crude reaction mixture using silica gel afforded (E)-4-(3-ethoxy-3-oxoprop-1-en-1-yl)phenyl 2-(dimethylamino)benzoate $4 d$ as a yellow viscous oil ( $0.106 \mathrm{~g}, 62 \%$ yield).

$\boldsymbol{R}_{\mathbf{f}}$ (Pet. ether $/$ EtOAc = 90/10): 0.27; ${ }^{1} \mathbf{H}$ NMR (400 MHz, $\left.\mathbf{C D C l}_{3}\right) \delta 7.96(\mathrm{~d}, J=7.7 \mathrm{~Hz}, 1 \mathrm{H})$, $7.71(\mathrm{~d}, J=16.0 \mathrm{~Hz}, 1 \mathrm{H}), 7.60(\mathrm{~d}, J=8.4 \mathrm{~Hz}, 2 \mathrm{H}), 7.45(\mathrm{t}, J=7.5 \mathrm{~Hz}, 1 \mathrm{H}), 7.27(\mathrm{~d}, J=8.3 \mathrm{~Hz}$, 2H), $7.03(\mathrm{~d}, J=8.4 \mathrm{~Hz}, 1 \mathrm{H}), 6.92(\mathrm{t}, J=7.5 \mathrm{~Hz}, 1 \mathrm{H}), 6.43(\mathrm{~d}, J=16.0 \mathrm{~Hz}, 1 \mathrm{H}), 4.29(\mathrm{q}, J=$ 
$7.1 \mathrm{~Hz}, 2 \mathrm{H}), 2.95(\mathrm{~s}, 6 \mathrm{H}), 1.36(\mathrm{t}, J=7.1 \mathrm{~Hz}, 3 \mathrm{H}) .{ }^{13} \mathbf{C} \mathbf{~ N M R}\left(\mathbf{1 0 0} \mathbf{~ M H z}, \mathbf{C D C l}_{\mathbf{3}}\right) \delta$ 167.05, $165.89,153.42,152.68,143.71,133.39,132.46,132.07,129.30,122.37,118.78,118.65,118.36$, 117.06, 60.65, 43.92, 14.42. HRMS (ESI) calculated $[\mathrm{M}+\mathrm{H}]^{+}$for $\mathrm{C}_{20} \mathrm{H}_{22} \mathrm{O}_{4} \mathrm{~N}$ : 340.1543 , found: 340.1544. FTIR (cm $\left.{ }^{-1}\right)$ : 3020, 1713, 1599, 1505, 1313, 1210, 1032, 761.

\section{(E)-4-(3-Oxoprop-1-en-1-yl)phenyl 2-(dimethylamino)benzoate (4e)}

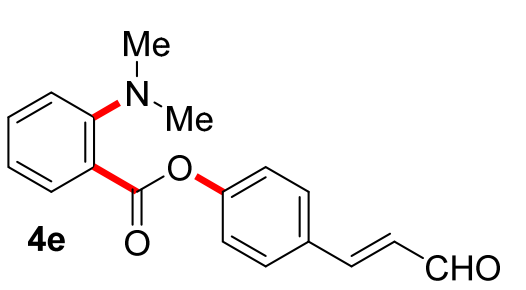

Following the general procedure, 2-(trimethylsilyl)phenyl
trifluoromethanesulfonate 1a $(0.179 \mathrm{~g}, 146 \mu \mathrm{L}, 0.60 \mathrm{mmol})$ was
treated with $(E)$-3-(4-(dimethylamino)phenyl)acrylaldehyde $2 \mathrm{~s}$
$(0.088 \mathrm{~g}, 0.50 \mathrm{mmol})$ in the presence of $\mathrm{KF}(0.070 \mathrm{~g}, 1.20$ mmol) and 18-crown-6 (0.317 g, $1.20 \mathrm{mmol})$ in THF $(2.0 \mathrm{~mL})$ under an atmosphere of $\mathrm{CO}_{2}$ (balloon pressure) at $-10{ }^{\circ} \mathrm{C}$ to $\mathrm{rt}$ for $12 \mathrm{~h}$. Evaporation of solvent followed by flash column chromatography (Pet. ether $/$ EtOAc $=85 / 15$ ) of the crude reaction mixture using silica gel afforded (E)-4-(3-oxoprop-1-en-1-yl)phenyl 2-(dimethylamino)benzoate $4 \mathbf{e}$ as a yellow viscous oil ( $0.096 \mathrm{~g}, 65 \%$ yield).

$\boldsymbol{R}_{\mathbf{f}}($ Pet. ether $/$ EtOAc $=70 / 30): 0.50 ;{ }^{1} \mathbf{H}$ NMR $\left(\mathbf{4 0 0} \mathbf{~ M H z}, \mathbf{C D C l}_{3}\right) \delta 9.70(\mathrm{~d}, J=7.7 \mathrm{~Hz}, 1 \mathrm{H})$, $7.95(\mathrm{~d}, J=7.7 \mathrm{~Hz}, 1 \mathrm{H}), 7.62(\mathrm{~d}, J=8.4 \mathrm{~Hz}, 2 \mathrm{H}), 7.50-7.42(\mathrm{~m}, 2 \mathrm{H}), 7.30(\mathrm{~d}, J=8.4 \mathrm{~Hz}, 2 \mathrm{H})$, $7.02(\mathrm{~d}, J=8.4 \mathrm{~Hz}, 1 \mathrm{H}), 6.91(\mathrm{t}, J=7.5 \mathrm{~Hz}, 1 \mathrm{H}), 6.69\left(\mathrm{dd}, J_{l}=15.9 \mathrm{~Hz}, J_{2}=7.6 \mathrm{~Hz}, 1 \mathrm{H}\right), 2.93$ (s, 6H). ${ }^{13} \mathbf{C}$ NMR (100 MHz, $\left.\mathbf{C D C l}_{3}\right) \delta$ 193.68, 165.67, 153.48, 151.82, 133.51, 132.48, 131.56, 129.82, 128.59, 122.64, 118.64, 118.49, 117.08, 43.91. HRMS (ESI) calculated $[\mathrm{M}+\mathrm{H}]^{+}$for $\mathrm{C}_{18} \mathrm{H}_{18} \mathrm{O}_{3} \mathrm{~N}: 296.1281$, found: 296.1279 . FTIR $\left(\mathbf{c m}^{-1}\right): 3018,2845,2741,1726,1676,1599$, $1502,1215,1165,1124,1030,770$.

\section{4-(Ethoxycarbonyl)phenyl 2-(dimethylamino)-4,5-dimethylbenzoate (4f)}

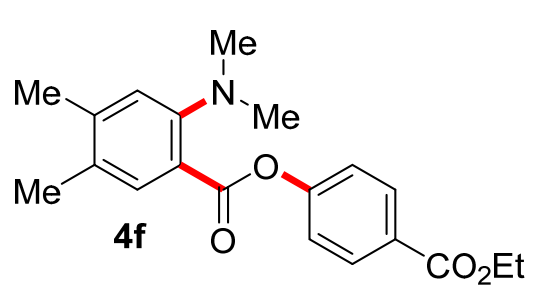

Following the general procedure, 4,5-dimethyl-2(trimethylsilyl)phenyl trifluoromethanesulfonate $\mathbf{1 b}(0.196 \mathrm{~g}$, $0.60 \mathrm{mmol}$ ) was treated with ethyl 4-(dimethylamino)benzoate $2 \mathrm{o}(0.097 \mathrm{~g}, 0.50 \mathrm{mmol})$ in the presence of $\mathrm{KF}(0.070 \mathrm{~g}, 1.20$ $\mathrm{mmol})$ and 18 -crown-6 (0.317 g, $1.20 \mathrm{mmol})$ in THF $(2.0 \mathrm{~mL})$ under an atmosphere of $\mathrm{CO}_{2}$ (balloon pressure) at $-10{ }^{\circ} \mathrm{C}$ for $24 \mathrm{~h}$. Evaporation of solvent followed by flash column chromatography $($ Pet. ether $/$ EtOAc $=95 / 05)$ of the crude reaction 
mixture using silica gel afforded 4-(ethoxycarbonyl)phenyl 2-(dimethylamino)-4,5dimethylbenzoate $\mathbf{4 f}$ as a yellow solid $(0.111 \mathrm{~g}, 65 \%$ yield).

$\boldsymbol{R}_{\mathbf{f}}($ Pet. ether $/$ EtOAc $=90 / 10): 0.38 ;{ }^{1} \mathbf{H}$ NMR $\left(\mathbf{4 0 0} \mathbf{~ M H z}, \mathbf{C D C l}_{3}\right) \delta 8.11(\mathrm{~d}, J=8.5 \mathrm{~Hz}, 2 \mathrm{H})$, 7.77 (s, 1H), 7.29 (d, $J=8.5 \mathrm{~Hz}, 2 \mathrm{H}), 6.84$ (s, 1H), 4.39 (q, $J=7.1 \mathrm{~Hz}, 2 \mathrm{H}), 2.88$ (s, 6H), 2.30 (s, 3H), 2.24 (s, 3H), 1.40 (t, $J=7.1 \mathrm{~Hz}, 3 \mathrm{H}) .{ }^{13} \mathbf{C}$ NMR (100 MHz, CDCl $) \delta$ 166.13, 165.21, 155.07, 152.46, 143.22, 133.36, 131.19, 127.79, 127.73, 121.92, 118.99, 116.74, 61.15, 44.42, 20.58, 18.80, 14.46. HRMS (ESI) calculated $[\mathrm{M}+\mathrm{Na}]^{+}$for $\mathrm{C}_{20} \mathrm{H}_{23} \mathrm{O}_{4} \mathrm{NNa}$ : 364.1519, found: 364.1512. FTIR (cm $\left.{ }^{-1}\right)$ : 3020, 1716, 1607, 1505, 1280, 1216, 1162, 1109, 1019, 772.

\section{4-(Ethoxycarbonyl)phenyl 6-(dimethylamino)benzo[d][1,3]dioxole-5-carboxylate (4g)}

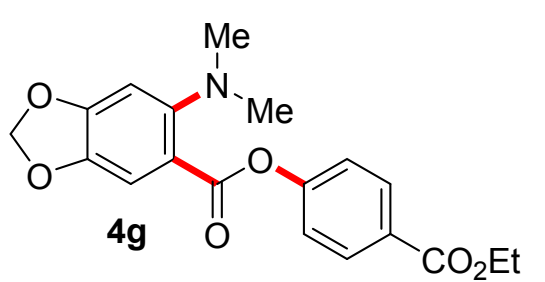

Following the general procedure, 6-(trimethylsilyl)benzo $[d]$ [1,3]dioxol-5-yl trifluoromethanesulfonate $1 \mathbf{c}(0.205 \mathrm{~g}, 0.60$ mmol) was treated with ethyl 4-(dimethylamino)benzoate 20 $(0.097 \mathrm{~g}, 0.50 \mathrm{mmol})$ in the presence of $\mathrm{KF}(0.070 \mathrm{~g}, 1.20$ $\mathrm{mmol})$ and 18 -crown-6 (0.317 g, $1.20 \mathrm{mmol})$ in THF $(2.0 \mathrm{~mL})$

under an atmosphere of $\mathrm{CO}_{2}$ (balloon pressure) at $-10{ }^{\circ} \mathrm{C}$ for $24 \mathrm{~h}$. Evaporation of solvent followed by flash column chromatography $($ Pet. ether $/$ EtOAc $=95 / 05)$ of the crude reaction mixture using silica gel afforded 4-(ethoxycarbonyl)phenyl 6-(dimethylamino)benzo[ $d]$ [1,3]dioxole-5-carboxylate $\mathbf{4 g}$ as a yellow viscous oil (0.108 $\mathrm{g}, 61 \%$ yield).

$\boldsymbol{R}_{\mathbf{f}}($ Pet. ether $/$ EtOAc $=90 / 10): 0.41 ;{ }^{1} \mathbf{H}$ NMR $\left(\mathbf{4 0 0} \mathbf{~ M H z}, \mathbf{C D C l}_{\mathbf{3}}\right) \delta 8.11(\mathrm{~d}, J=8.4 \mathrm{~Hz}, 2 \mathrm{H})$, $7.52(\mathrm{~s}, 1 \mathrm{H}), 7.28$ (d, $J=8.4 \mathrm{~Hz}, 2 \mathrm{H}), 6.63(\mathrm{~s}, 1 \mathrm{H}), 5.99$ (d, $J=1.0 \mathrm{~Hz}, 2 \mathrm{H}), 4.39$ (q, $J=7.0 \mathrm{~Hz}$, 2H), $2.87(\mathrm{~s}, 6 \mathrm{H}), 1.41(\mathrm{t}, J=7.1 \mathrm{~Hz}, 3 \mathrm{H}) .{ }^{13} \mathbf{C} \mathbf{~ N M R}\left(\mathbf{1 0 0} \mathbf{M H z}, \mathbf{C D C l}_{3}\right) \delta 166.03,163.73$, 154.98, 153.12, 152.56, 140.91, 131.11, 127.68, 121.86, 111.07, 110.90, 101.84, 99.15, 61.07, 44.78, 14.39. HRMS (ESI) calculated $[\mathrm{M}+\mathrm{H}]^{+}$for $\mathrm{C}_{19} \mathrm{H}_{20} \mathrm{O}_{6} \mathrm{~N}$ : 358.1285, found: 358.1286. FTIR (cm $\left.{ }^{-1}\right)$ : 3020, 2902, 2796, 2404, 1712, 1604, 1492, 1421, 1215, 1100, 759.

\section{4-(Ethoxycarbonyl)phenyl 2-(dimethylamino)-4,5-difluorobenzoate (4h)}

Following the general procedure, 4,5-difluoro-2-(trimethylsilyl)phenyl trifluoromethanesulfonate 1 d $(0.200 \mathrm{~g}, 0.60 \mathrm{mmol})$ was treated with ethyl 4-(dimethylamino)benzoate $2 \mathrm{o}(0.097 \mathrm{~g}, 0.50$ $\mathrm{mmol})$ in the presence of KF $(0.070 \mathrm{~g}, 1.20 \mathrm{mmol})$ and 18 -crown-6 $(0.317 \mathrm{~g}, 1.20 \mathrm{mmol})$ in THF $(2.0 \mathrm{~mL})$ under an atmosphere of $\mathrm{CO}_{2}$ (balloon pressure) at $-10{ }^{\circ} \mathrm{C}$ for $24 \mathrm{~h}$. Evaporation of 


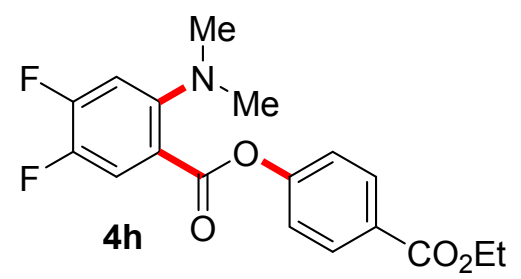

solvent followed by flash column chromatography (Pet. ether $/$ EtOAc $=95 / 05)$ of the crude reaction mixture using silica gel afforded 4-(ethoxycarbonyl) phenyl 2-(dimethylamino)-4,5difluorobenzoate $4 \mathrm{~h}$ as a yellow viscous oil $(0.105 \mathrm{~g}, 60 \%$ yield).

$\boldsymbol{R}_{\mathbf{f}}($ Pet. ether $/$ EtOAc $=90 / 10): 0.47 ;{ }^{1} \mathbf{H}$ NMR (400 MHz, $\left.\mathbf{C D C l}_{3}\right) \delta 8.14(\mathrm{~d}, J=8.4 \mathrm{~Hz}, 2 \mathrm{H})$, $7.86(\mathrm{t}, J=10.0 \mathrm{~Hz}, 1 \mathrm{H}), 7.29(\mathrm{~d}, J=8.2 \mathrm{~Hz}, 2 \mathrm{H}), 6.82\left(\mathrm{dd}, J_{1}=13.0, J_{2}=6.7 \mathrm{~Hz}, 1 \mathrm{H}\right), 4.41(\mathrm{q}, J$ $=7.1 \mathrm{~Hz}, 2 \mathrm{H}), 2.91(\mathrm{~s}, 6 \mathrm{H}), 1.42(\mathrm{t}, J=7.1 \mathrm{~Hz}, 3 \mathrm{H}) .{ }^{13} \mathbf{C}$ NMR (100 MHz, CDCl $\left.\mathbf{3}\right) \delta 165.99$, $163.33\left(\mathrm{dd}, J_{l}=253.2, J_{2}=13.8 \mathrm{~Hz}\right), 151.94(\mathrm{~d}, J=8.9 \mathrm{~Hz}), 143.33\left(\mathrm{dd}, J_{l}=240.9, J_{2}=13.1\right.$ Hz), 131.91, 131.33, 128.24, 121.72, 121.10 (d, $J=19.3 \mathrm{~Hz}), 106.21$ (d, $J=19.8 \mathrm{~Hz}), 61.26$, 44.25, 14.45. HRMS (ESI) calculated $[\mathrm{M}+\mathrm{H}]^{+}$for $\mathrm{C}_{18} \mathrm{H}_{18} \mathrm{O}_{4} \mathrm{NF}_{2}: 350.1198$, found: 350.1198 . FTIR (cm $\left.{ }^{-1}\right):$ 3022, 2951, 2256, 1722, 1598, 1517, 1445, 1362, 1261, 1082, 952.

\section{4-(Ethoxycarbonyl)phenyl 3-(dimethylamino)-2-naphthoate (4i)}<smiles>CCOC(=O)c1ccc(OC(=O)c2cc3ccccc3cc2N(C)C)cc1</smiles>

Following the general procedure, 3-(trimethylsilyl)naphthalen2-yl trifluoromethanesulfonate 1e (0.209 g, $0.60 \mathrm{mmol})$ was treated with ethyl 4-(dimethylamino)benzoate 20 (0.097 g, $0.50 \mathrm{mmol})$ in the presence of $\mathrm{KF}(0.070 \mathrm{~g}, 1.20 \mathrm{mmol})$ and 18-crown-6 (0.317 g, $1.20 \mathrm{mmol})$ in THF $(2.0 \mathrm{~mL})$ under an

atmosphere of $\mathrm{CO}_{2}$ (balloon pressure) at $-10{ }^{\circ} \mathrm{C}$ for $24 \mathrm{~h}$. Evaporation of solvent followed by flash column chromatography (Pet. ether $/$ EtOAc $=95 / 05)$ of the crude reaction mixture using silica gel afforded 4-(ethoxycarbonyl)phenyl 3-(dimethylamino)-2-naphthoate 4i as a yellow viscous oil ( $0.106 \mathrm{~g}, 58 \%$ yield).

$\boldsymbol{R}_{\mathbf{f}}($ Pet. ether $/$ EtOAc $=90 / 10): 0.53 ;{ }^{1} \mathbf{H}$ NMR (400 MHz, $\left.\mathbf{C D C l}_{3}\right) \delta 8.48(\mathrm{~s}, 1 \mathrm{H}), 8.16(\mathrm{~d}, J=$ $8.5 \mathrm{~Hz}, 2 \mathrm{H}), 7.82(\mathrm{~d}, J=8.1 \mathrm{~Hz}, 1 \mathrm{H}), 7.74(\mathrm{~d}, J=8.3 \mathrm{~Hz}, 1 \mathrm{H}), 7.52$ (t, $J=7.5 \mathrm{~Hz}, 1 \mathrm{H}), 7.38-$ $7.36(\mathrm{~m}, 3 \mathrm{H}), 7.31(\mathrm{~s}, 1 \mathrm{H}), 4.41$ (q, $J=7.1 \mathrm{~Hz}, 2 \mathrm{H}), 2.98(\mathrm{~s}, 6 \mathrm{H}), 1.42(\mathrm{t}, J=7.1 \mathrm{~Hz}, 3 \mathrm{H}) .{ }^{13} \mathbf{C}$ NMR (100 MHz, $\left.\mathbf{C D C l}_{3}\right) \delta 166.05,165.86,154.80,149.54,136.38,133.80,131.32,128.83$, $128.78,128.16,127.53,126.59,124.42,122.81,121.81,113.39,61.24,44.45,14.46$. HRMS (ESI) calculated $[\mathrm{M}+\mathrm{H}]^{+}$for $\mathrm{C}_{22} \mathrm{H}_{22} \mathrm{O}_{4} \mathrm{~N}: 364.1543$, found: 364.1534 . FTIR (cm $\left.{ }^{-1}\right): 3021,2982$, $2935,2360,1746,1642,1513,1418,1186,1026,816,756$. 


\section{4-(Ethoxycarbonyl)phenyl 2-(dimethylamino)-1-naphthoate (4j)}<smiles>CCOC(=O)c1ccc(OC(=O)c2c(N(C)C)ccc3ccccc23)cc1</smiles>

4j

Following the general procedure, 1-(trimethylsilyl)naphthalen-2yl trifluoromethanesulfonate $1 \mathbf{f}(0.209 \mathrm{~g}, 0.60 \mathrm{mmol})$ was treated with ethyl 4-(dimethylamino)benzoate $20(0.097 \mathrm{~g}, 0.50 \mathrm{mmol})$ in the presence of KF (0.070 g, $1.20 \mathrm{mmol})$ and 18-crown-6 (0.317 $\mathrm{g}, 1.20 \mathrm{mmol})$ in THF $(2.0 \mathrm{~mL})$ under an atmosphere of $\mathrm{CO}_{2}$ (balloon pressure) at $-10{ }^{\circ} \mathrm{C}$ for $24 \mathrm{~h}$. Evaporation of solvent followed by flash column chromatography (Pet. ether $/$ EtOAc $=95 / 05)$ of the crude reaction mixture using silica gel afforded 4-(ethoxycarbonyl)phenyl 2-(dimethylamino)-1-naphthoate $\mathbf{4 j}$ as a yellow viscous oil (0.113 g, 62\% yield).

$\boldsymbol{R}_{\mathbf{f}}($ Pet. ether $/$ EtOAc $=90 / 10): 0.53 ;{ }^{1} \mathbf{H}$ NMR (400 MHz, $\left.\mathbf{C D C l}_{3}\right) \delta 8.17(\mathrm{~d}, J=8.5 \mathrm{~Hz}, 2 \mathrm{H})$, $7.99(\mathrm{~d}, J=8.5 \mathrm{~Hz}, 1 \mathrm{H}), 7.87$ (d, $J=8.9 \mathrm{~Hz}, 1 \mathrm{H}), 7.80(\mathrm{~d}, J=7.9 \mathrm{~Hz}, 1 \mathrm{H}), 7.54$ (t, $J=7.6 \mathrm{~Hz}$, 1H), 7.44-7.36 (m, 4H), 4.41 (q, $J=7.1 \mathrm{~Hz}, 2 \mathrm{H}), 3.02(\mathrm{~s}, 6 \mathrm{H}), 1.42(\mathrm{t}, J=7.1 \mathrm{~Hz}, 3 \mathrm{H}) .{ }^{13} \mathbf{C}$ NMR (100 MHz, $\left.\mathbf{C D C l}_{3}\right) \delta$ 167.80, 165.97, 154.73, 149.78, 131.67, 131.33, 128.90, 128.32, $128.20,127.82,124.27,123.24,121.68,118.91,118.73,61.21,44.34,14.44$. HRMS (ESI) calculated $[\mathrm{M}+\mathrm{H}]^{+}$for $\mathrm{C}_{22} \mathrm{H}_{22} \mathrm{O}_{4} \mathrm{~N}: 364.1543$, found: 364.1535. FTIR (cm $\left.{ }^{-1}\right): 3017,2983,2930$, 2401, 2360, 1730, 1590, 1444, 1292, 1120, 993, 769.

\section{4-(Ethoxycarbonyl)phenyl 2-(dimethylamino)-5-methylbenzoate and}

\section{4-(Ethoxycarbonyl)phenyl 2-(dimethylamino)-4-methylbenzoate (4k and 4k')}

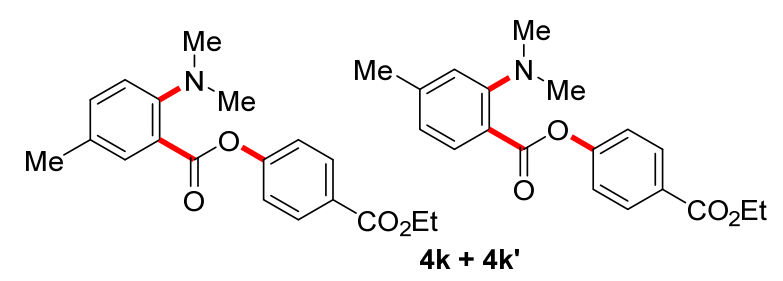

Following the general procedure, 4-methyl-2(trimethylsilyl)phenyl trifluoromethanesulfonate $1 \mathrm{~g}(0.187 \mathrm{~g}, 0.60 \mathrm{mmol})$ was treated with ethyl 4(dimethylamino)benzoate $\mathbf{2 0} \quad(0.097 \mathrm{~g}, \quad 0.50$

$\mathrm{mmol})$ in the presence of $\mathrm{KF}(0.070 \mathrm{~g}, 1.20 \mathrm{mmol})$ and 18 -crown-6 $(0.317 \mathrm{~g}, 1.20 \mathrm{mmol})$ in THF $\left(2.0 \mathrm{~mL}\right.$ ) under an atmosphere of $\mathrm{CO}_{2}$ (balloon pressure) at $-10{ }^{\circ} \mathrm{C}$ for $24 \mathrm{~h}$. Evaporation of solvent followed by flash column chromatography (Pet. ether $/$ EtOAc $=95 / 05)$ of the crude reaction mixture using silica gel afforded 4-(ethoxycarbonyl)phenyl 2-(dimethylamino)-5methylbenzoate $\mathbf{4 k}$ and 4-(ethoxycarbonyl)phenyl 2-(dimethylamino)-4-methylbenzoate $\mathbf{4 k}$ ' as a mixture of regioisomers in 1.7:1 ratio $(0.100 \mathrm{~g}, 61 \%$ yield, yellow viscous oil, regioisomer ratio determined by ${ }^{1} \mathrm{H}-\mathrm{NMR}$ analysis of crude reaction mixture). 
$\boldsymbol{R}_{\mathbf{f}}($ Pet. ether $/$ EtOAc $=90 / 10): 0.51 ;{ }^{1} \mathbf{H}$ NMR $\left(\mathbf{4 0 0} \mathbf{~ M H z}, \mathbf{C D C l}_{3}\right) \delta 8.15-8.12(\mathrm{~m}, 2 \mathrm{H}), 7.92(\mathrm{~d}$, $J=8.0 \mathrm{~Hz}, 1 \mathrm{H}), 7.33-7.27(\mathrm{~m}, 2 \mathrm{H}), 6.84(\mathrm{~s}, 1 \mathrm{H}), 6.75(\mathrm{~d}, J=8.0 \mathrm{~Hz}, 1 \mathrm{H}), 4.40(\mathrm{q}, J=7.1 \mathrm{~Hz}$, 2H), 2.94 (s, 6H), 2.40 (s, 3H), 1.42 (t, $J=7.0 \mathrm{~Hz}, 3 \mathrm{H}) .{ }^{13} \mathbf{C} \mathbf{~ N M R}\left(\mathbf{1 0 0} \mathbf{~ M H z}, \mathbf{C D C l}_{\mathbf{3}}\right) \delta 166.02$, $166.19,154.99,153.92,144.40,134.29,132.77,131.14,127.75,121.81,119.88,117.71,61.08$, 43.99, 22.05, 14.40. Representative peaks of the minor isomer ${ }^{1} \mathbf{H}$ NMR $\left(400 \mathbf{~ M H z}, \mathbf{C D C l}_{3}\right) \delta$ 8.15-8.12 (m, 2H), 7.78 (s, 1H), 7.33-7.27 (m, 3H), 6.97 (d, $J=8.4 \mathrm{~Hz}, 1 \mathrm{H}), 2.91$ (s, 3H), 2.34 (s, 3H). ${ }^{13} \mathbf{C}$ NMR (100 MHz, $\left.\mathbf{C D C l}_{3}\right) \delta$ 165.56, 154.90, 151.70, 132.46, 131.17, 128.60, 127.87, 117.56, 115.80. HRMS (ESI) calculated $[\mathrm{M}+\mathrm{H}]^{+}$for $\mathrm{C}_{19} \mathrm{H}_{22} \mathrm{O}_{4} \mathrm{~N}$ : 328.1537, found: 328.1537 . FTIR $\left(\mathbf{c m}^{-1}\right)$ : 3019, 2983, 2400, 2335, 1843, 1744, 1642, 1612, 1418, 1374, 1218, 995, 759.

\section{Synthesis and Characterization of 10-Methylacridin-9(10H)-one (10) ${ }^{7}$}

\section{i) Reduction of Methyl 2-(methyl(phenyl)amino)benzoate (3a)}

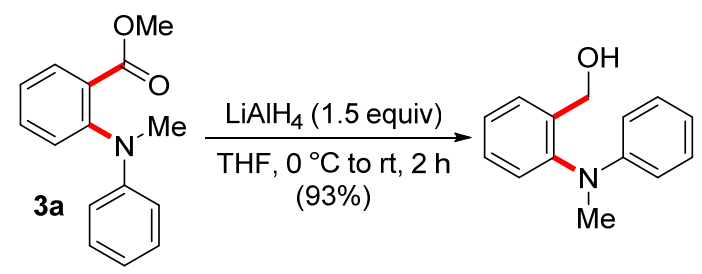

A solution of methyl 2-(methyl(phenyl)amino)benzoate $3 \mathbf{a}(0.200 \mathrm{~g}, 0.83 \mathrm{mmol})$ in THF $(4 \mathrm{~mL})$ was added dropwise at $0{ }^{\circ} \mathrm{C}$ to a stirred solution of $\mathrm{LiAlH}_{4}(0.047 \mathrm{~g}, 1.25 \mathrm{mmol})$ in THF $(1 \mathrm{~mL})$. After $2 \mathrm{~h}$, EtOAc $(2 \mathrm{ml})$ and then water $(2 \mathrm{ml})$ were added cautiously to the solution. After filtration and removal of the solvent, the residue was extracted with EtOAc $(2 \mathrm{X} 5 \mathrm{~mL})$, and the organic phase was dried over anhydrous $\mathrm{Na}_{2} \mathrm{SO}_{4}$. The solvent was then evaporated to give the (2-(methyl(phenyl)amino)phenyl)methanol as oil $(0.164,93 \%$ yield), which was used in the next oxidation step without further purification.

ii) Synthesis of 2-(Methyl(phenyl)amino)benzaldehyde (9)

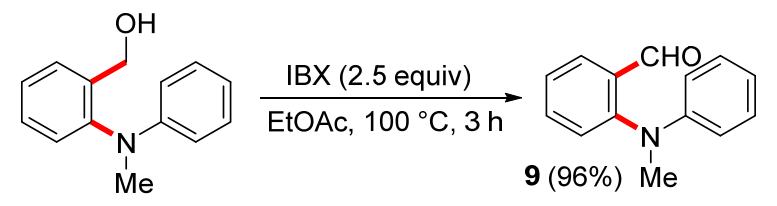

To a suspension of IBX (0.539 $\mathrm{g}, 1.93 \mathrm{mmol})$ in EtOAc $(8 \mathrm{~mL})$ was added a solution of (2-(methyl(phenyl)amino)phenyl)methanol $(0.164 \mathrm{~g}, 0.77 \mathrm{mmol})$ in EtOAc $(8 \mathrm{~mL})$. The reaction mixture was heated at $100{ }^{\circ} \mathrm{C}$ for $3 \mathrm{~h}$, cooled and filtered; the filtered solid was washed with

\footnotetext{
${ }^{7}$ Zheng, Z.; Dian, L.; Yuan, Y.; Zhang-Negrerie, D.; Du, Y.; Zhao, K. J.Org. Chem. 2014, 79, 7451.
} 
EtOAc $(2 \times 5 \mathrm{~mL})$. The combined filtrate and washings were evaporated under reduced pressure and purified by flash column chromatography (Pet. ether $/$ EtOAc $=98 / 02$ ), to give the 2 (methyl(phenyl)amino)benzaldehyde 9 as a yellow viscous oil ( $0.155 \mathrm{~g}, 96 \%$ yield).

2-(Methyl(phenyl)amino)benzaldehyde (9) ${ }^{7}$

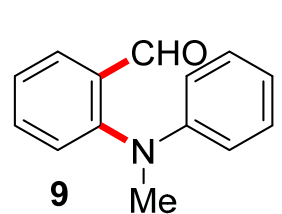

$\boldsymbol{R}_{\mathbf{f}}\left(\right.$ Pet. ether $/$ EtOAc $=$ 95/05): 0.48; ${ }^{1} \mathbf{H}$ NMR $\left(\mathbf{4 0 0} \mathbf{~ M H z}, \mathbf{C D C l}_{\mathbf{3}}\right) \delta 10.15(\mathrm{~s}$, $1 \mathrm{H}), 7.95(\mathrm{~d}, J=7.8 \mathrm{~Hz}, 1 \mathrm{H}), 7.64(\mathrm{t}, J=7.6 \mathrm{~Hz}, 1 \mathrm{H}), 7.35(\mathrm{t}, J=7.5 \mathrm{~Hz}$, $1 \mathrm{H}), 7.27$ (d, $J=7.6 \mathrm{~Hz}, 1 \mathrm{H}), 7.21$ (t, $J=7.8 \mathrm{~Hz}, 2 \mathrm{H}), 6.83(\mathrm{t}, J=7.3 \mathrm{~Hz}$, 1H), $6.74(\mathrm{~d}, J=8.1 \mathrm{~Hz}, 2 \mathrm{H}), 3.37$ (s, 3H). ${ }^{13} \mathbf{C}$ NMR (100 MHz, $\left.\mathbf{C D C l}_{3}\right) \delta$ 191.46, 152.15, 150.20, 135.95, 132.89, 129.40, 129.07, 127.86, 126.15, 119.29, 115.31, 41.67. HRMS (ESI) calculated $[\mathrm{M}+\mathrm{H}]^{+}$for $\mathrm{C}_{14} \mathrm{H}_{14} \mathrm{ON}: 212.1070$, found: 212.1069 . FTIR $\left(\mathbf{c m}^{-1}\right): 3020,1688,1596$, $1498,1216,772$.

\section{iii) Preparation of 10-Methylacridin-9(10H)-one (10)}

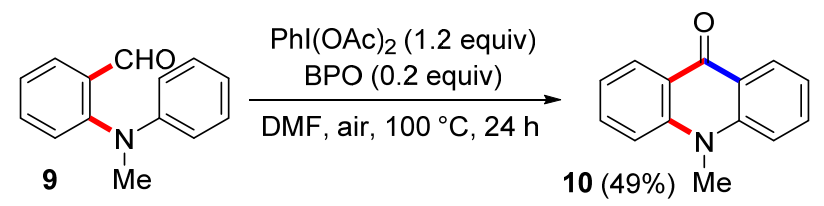

To a solution of 2-(methyl(phenyl)amino)benzaldehyde $9(0.146 \mathrm{~g}, 0.70 \mathrm{mmol})$ in DMF $(7 \mathrm{~mL})$ was added PIDA $(0.270 \mathrm{~g}, 0.84 \mathrm{mmol})$ and benzoyl peroxide $(0.034 \mathrm{~g}, 0.14 \mathrm{mmol})$. The resulting mixture was heated to $100{ }^{\circ} \mathrm{C}$ with vigorous stirring for $24 \mathrm{~h}$. The reaction mixture was cooled to room temperature, diluted with water $(25 \mathrm{~mL})$ and then extracted with EtOAc $(2 \mathrm{X} 20$ $\mathrm{mL})$. The organic layers were combined, washed with brine $(20 \mathrm{~mL})$, and dried over anhydrous $\mathrm{Na}_{2} \mathrm{SO}_{4}$. The solvent was then evaporated under reduced pressure, and the residue was purified by flash column chromatography (Pet. ether $/ \mathrm{EtOAc}=80 / 20)$ using silica gel to afford 10methylacridin- $9(10 H)$-one 10 as a light yellow solid ( $0.070 \mathrm{~g}, 49 \%$ yield).

10-Methylacridin-9(10H)-one (10) ${ }^{7}$

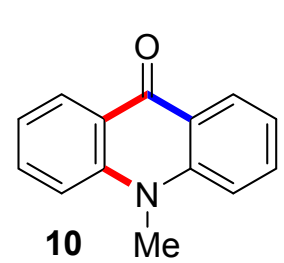

$\boldsymbol{R}_{\mathbf{f}}($ Pet. ether $/$ EtOAc $=70 / 30): 0.42 ;{ }^{1} \mathbf{H}$ NMR $\left(\mathbf{4 0 0} \mathbf{~ M H z}, \mathbf{C D C l}_{\mathbf{3}}\right) \delta 8.49(\mathrm{~d}$, $J=7.9 \mathrm{~Hz}, 2 \mathrm{H}), 7.64(\mathrm{t}, J=7.4 \mathrm{~Hz}, 2 \mathrm{H}), 7.41(\mathrm{~d}, J=8.7 \mathrm{~Hz}, 2 \mathrm{H}), 7.22(\mathrm{t}, J$ $=7.4 \mathrm{~Hz}, 2 \mathrm{H}), 3.77(\mathrm{~s}, 3 \mathrm{H}) .{ }^{13} \mathbf{C}$ NMR (100 MHz, $\left.\mathbf{C D C l}_{3}\right) \delta 178.12,142.51$, 133.80, 127.66, 122.45, 121.24, 114.82, 33.60. HRMS (ESI) calculated $[\mathrm{M}+\mathrm{H}]^{+}$for $\mathrm{C}_{14} \mathrm{H}_{12} \mathrm{ON}: 210.0913$, found: 210.0914. FTIR $\left(\mathbf{c m}^{-1}\right): 3419,3018,1697,1631,1600$, $1499,1461,1292,1216,1178,772$. 


\section{Synthesis and Characterization of 1-Phenyl-1,2-dihydro-4H-benzo[d] $[1,3]$ o xazin-4-one (12)}

i) Preparation of 2-(Methyl(phenyl)amino)benzoic acid (11)

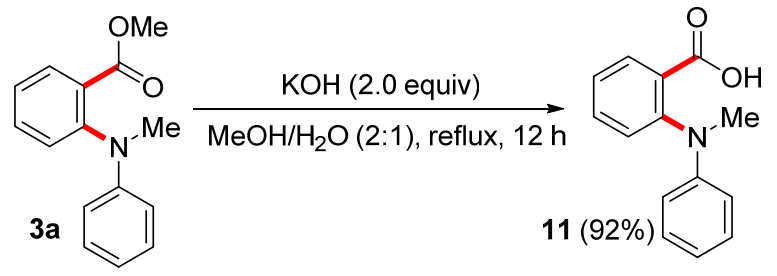

To a solution of methyl 2-(methyl(phenyl)amino)benzoate 3a $(0.200 \mathrm{~g}, 0.83 \mathrm{mmol})$ in $\mathrm{MeOH} / \mathrm{H}_{2} \mathrm{O}(6 \mathrm{~mL}, \mathrm{v}: \mathrm{v}=2: 1)$ was added $\mathrm{KOH}(0.093 \mathrm{~g}, 1.66 \mathrm{mmol})$. The resulting mixture was heated under reflux for $12 \mathrm{~h}$. The reaction mixture was subsequently allowed to cool at $\mathrm{rt}$. The solvent was removed under vacuum. The residue was diluted with water $(3 \mathrm{~mL})$, and acidified with $3 \mathrm{~N} \mathrm{HCl}(\mathrm{aq})$ to $\mathrm{pH}=2$. The precipitate was collected and dried under reduced pressure to afford the 2-(methyl(phenyl)amino)benzoic acid 11 as a yellow solid $(0.173 \mathrm{~g}, 92 \%$ yield).

\section{2-(Methyl(phenyl)amino)benzoic acid (11) ${ }^{8}$}

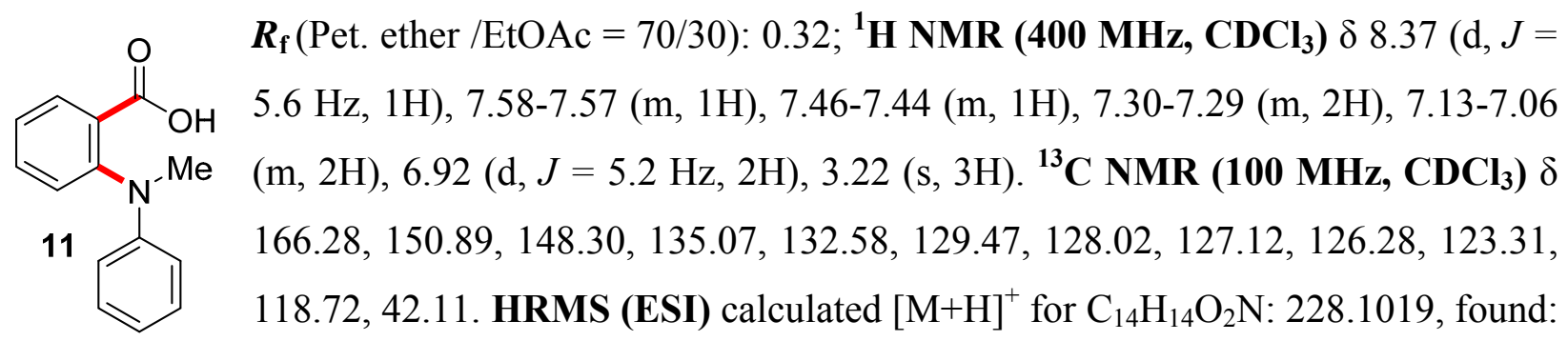
228.1017. FTIR (cm $\left.{ }^{-1}\right): 3020,1718,1599,1496,1456,1216,772$.

ii) Procedure for the PIDA/NaN ${ }_{3}-$ Mediated Oxygenation of the Benzoic Acid (11)

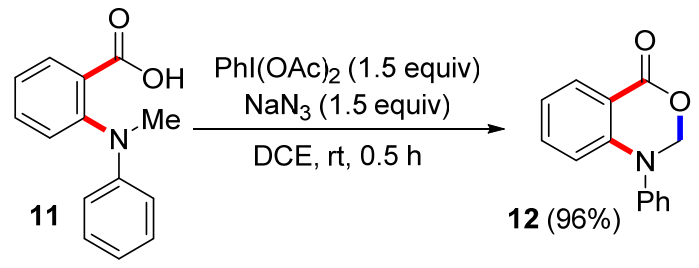

To a solution of 2-(methyl(phenyl)amino)benzoic acid $11(0.057 \mathrm{~g}, 0.25 \mathrm{mmol})$ in DCE $(1.20 \mathrm{~mL})$ was added PIDA $(0.122 \mathrm{~g}, 0.38 \mathrm{mmol})$ and $\mathrm{NaN}_{3}(0.025 \mathrm{~g}, 0.38 \mathrm{mmol})$. The mixture was stirred at $\mathrm{rt}$ for $0.5 \mathrm{~h}$. Then, saturated $\mathrm{NaHCO}_{3}(10 \mathrm{~mL})$ was added to quench the reaction,

${ }^{8}$ Zhang, N.; Cheng, R.; Zhang-Negrerie, D.; Du, Y.; Zhao, K. J. Org. Chem. 2014, 79, 10581. 
followed by extraction with EtOAc $(2 \times 10 \mathrm{~mL})$. The combined organic phase was dried over anhydrous $\mathrm{Na}_{2} \mathrm{SO}_{4}$, and filtered. The solvent was then evaporated to give the 1-phenyl-1,2dihydro-4H-benzo[ $d][1,3]$ oxazin-4-one 12 as a light brown viscous oil $(0.054,96 \%$ yield).

\section{1-Phenyl-1,2-dihydro-4H-benzo[d][1,3]oxazin-4-one (12) ${ }^{8}$}

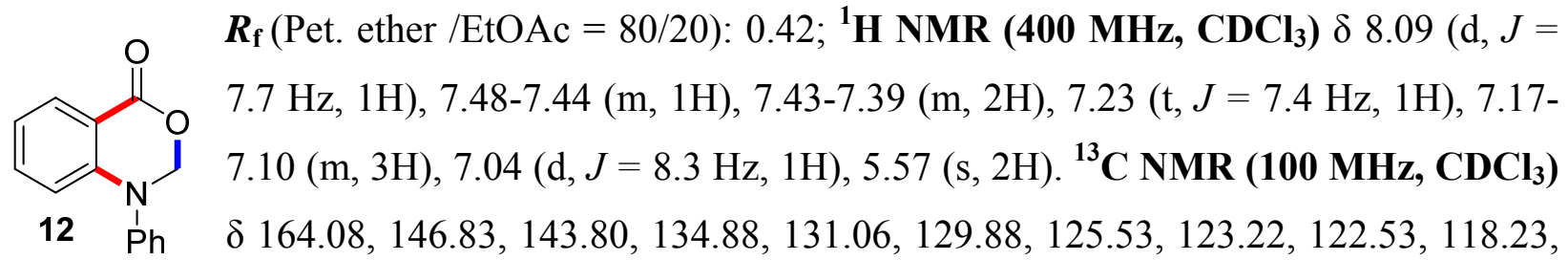

116.75, 80.77. HRMS (ESI) calculated $[\mathrm{M}+\mathrm{H}]^{+}$for $\mathrm{C}_{14} \mathrm{H}_{12} \mathrm{O}_{2} \mathrm{~N}$ : 226.0863, found: 226.0860 . FTIR $\left(\mathbf{c m}^{-1}\right): 3017,2108,1728,1608,1489,1362,1221,758$. 
9. ${ }^{1} \mathrm{H}$ and ${ }^{13} \mathrm{C}$ NMR Spectra of 2-Arylamino Benzoate and 2-Aminoaryl Benzoate Derivatives

Methyl 2-(methyl(phenyl)amino)benzoate (3a)

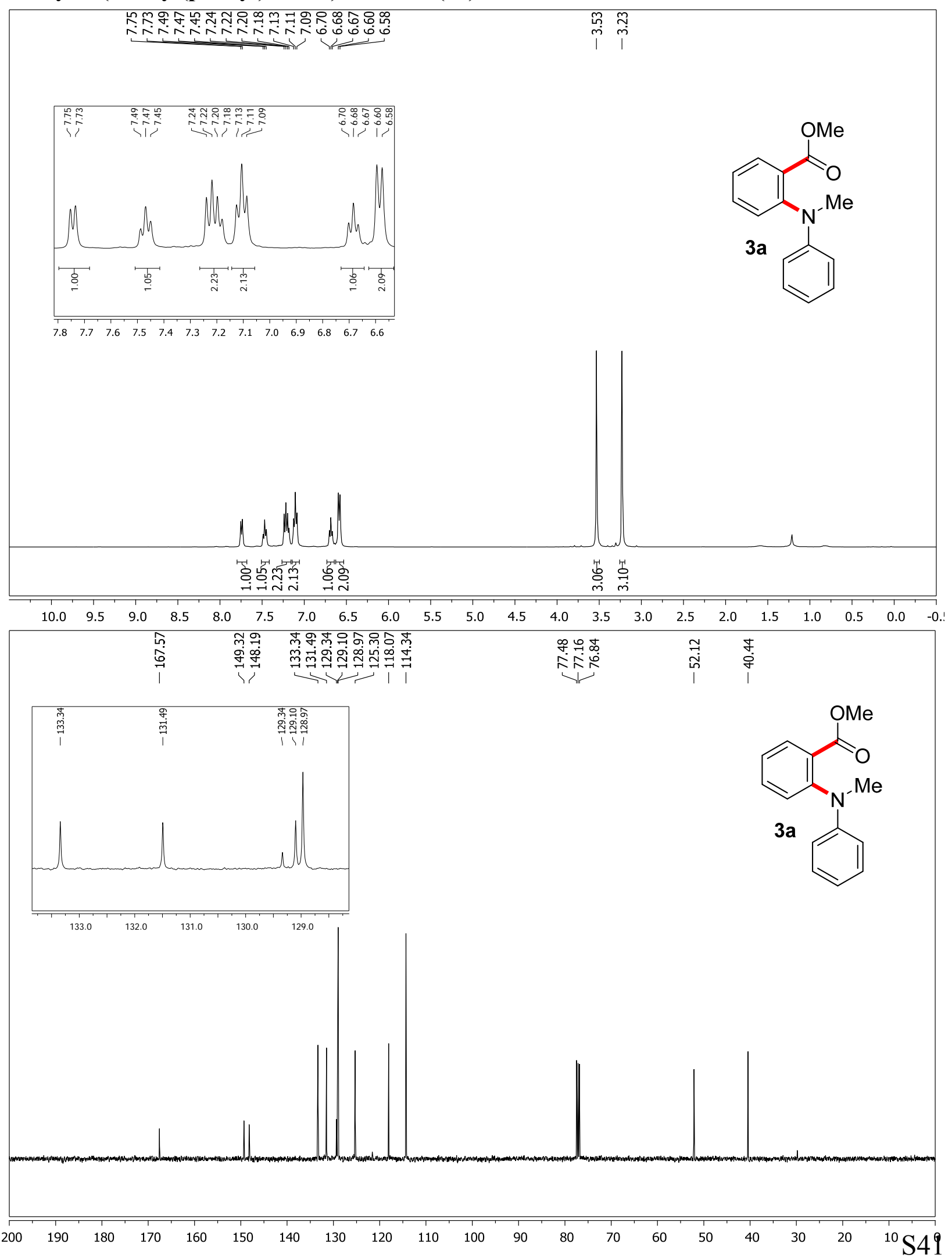


Methyl 2-(methyl(p-tolyl)amino)benzoate (3b)
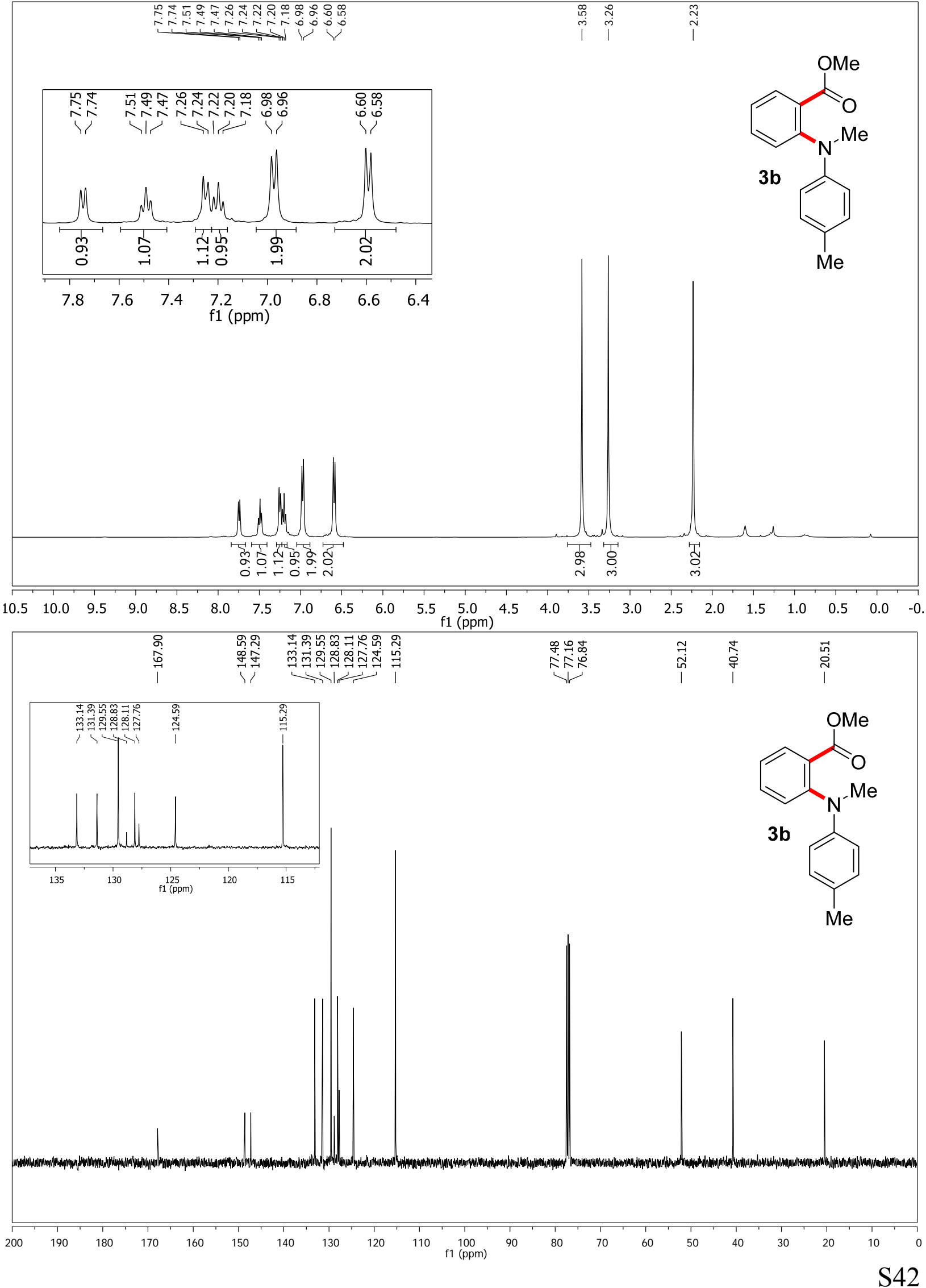
Methyl 2-((4-methoxyphenyl)(methyl)amino)benzoate (3c)

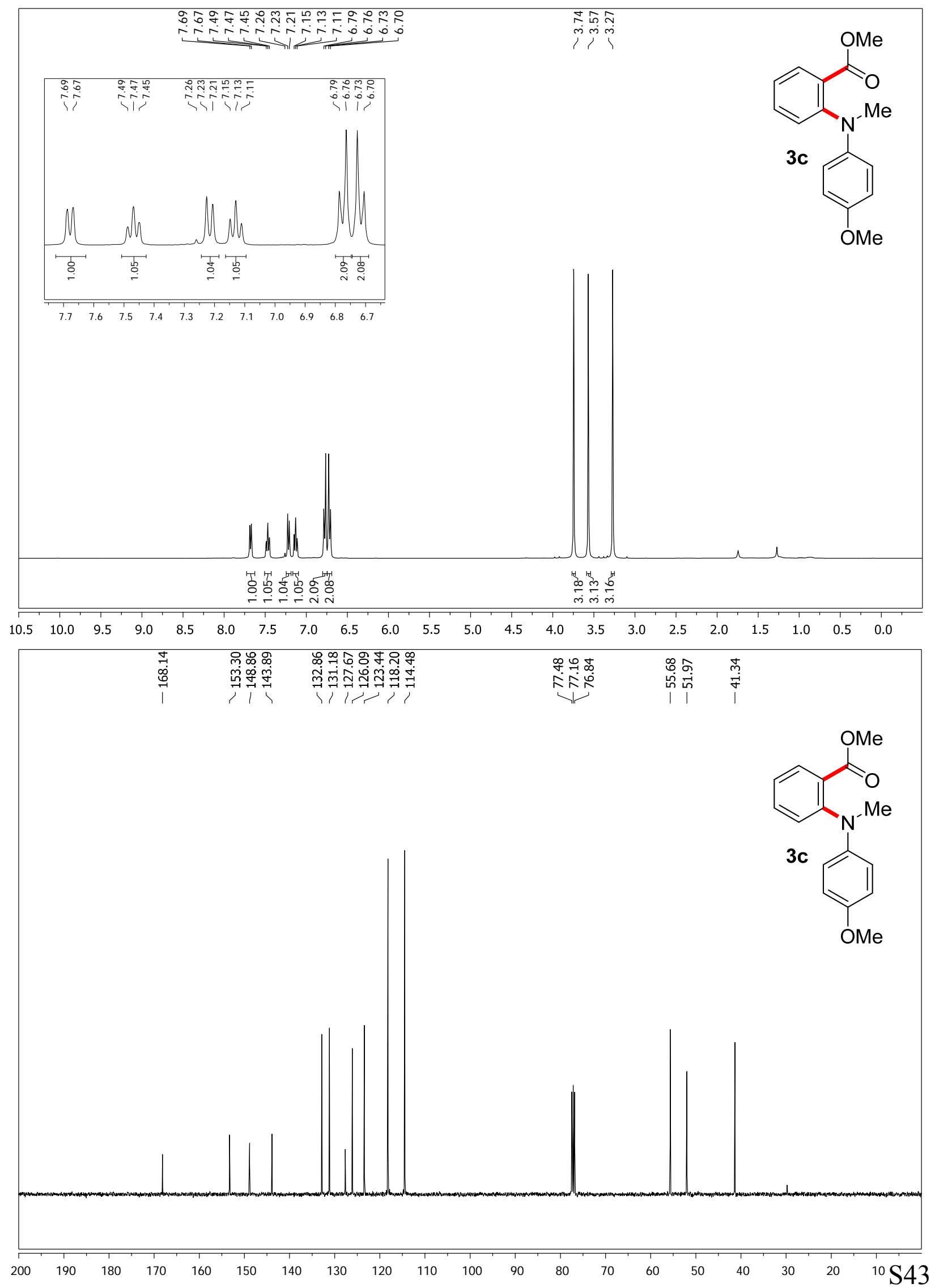


Methyl 2-((4-fluorophenyl)(methyl)amino)benzoate (3d)

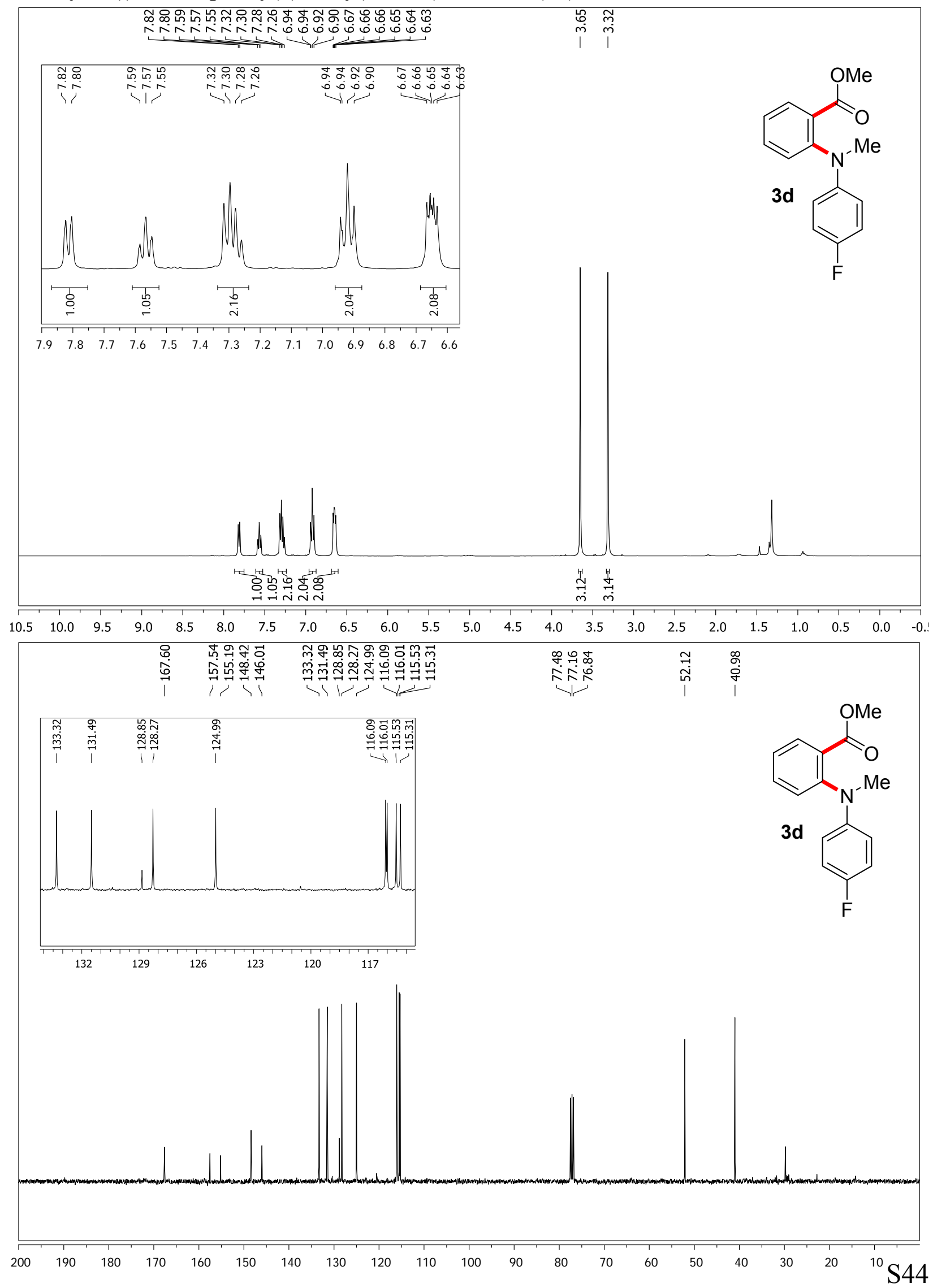


Methyl 2-(methyl(m-tolyl)amino)benzoate (3e)

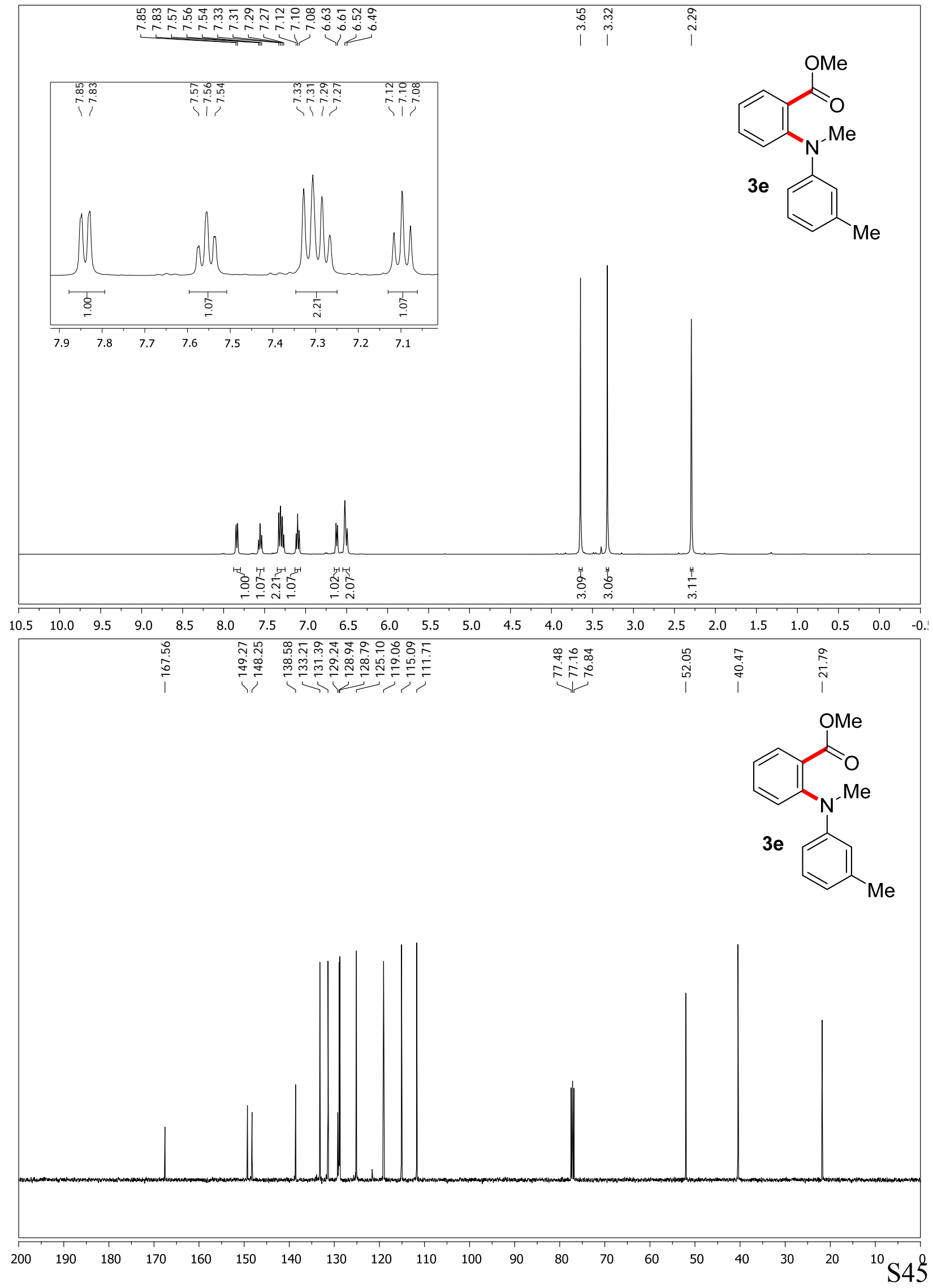


Methyl 2-((3-bromophenyl)(methyl)amino)benzoate (3f)
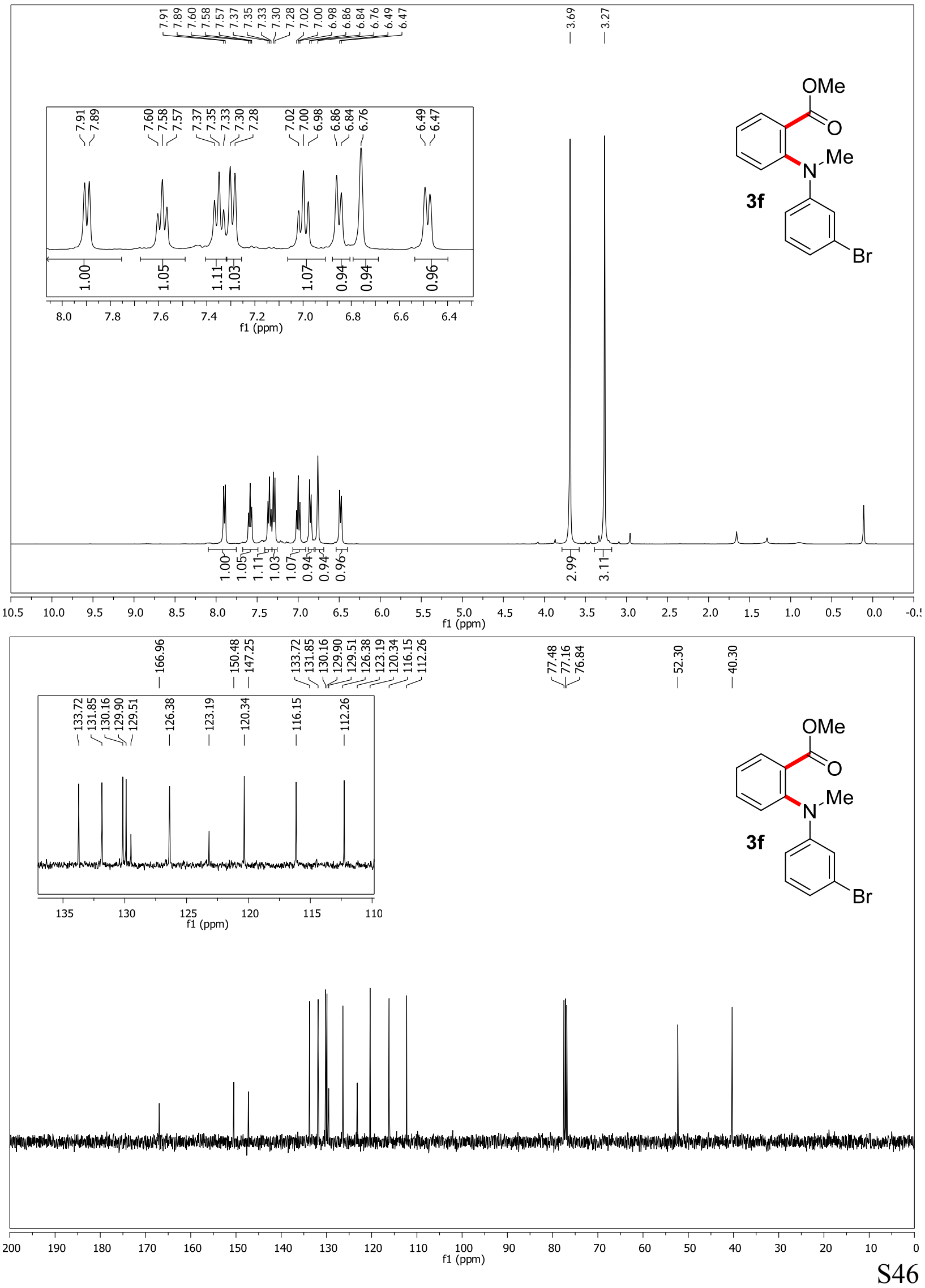
Dimethyl 2,2'-(methylazanediyl)dibenzoate (3g)

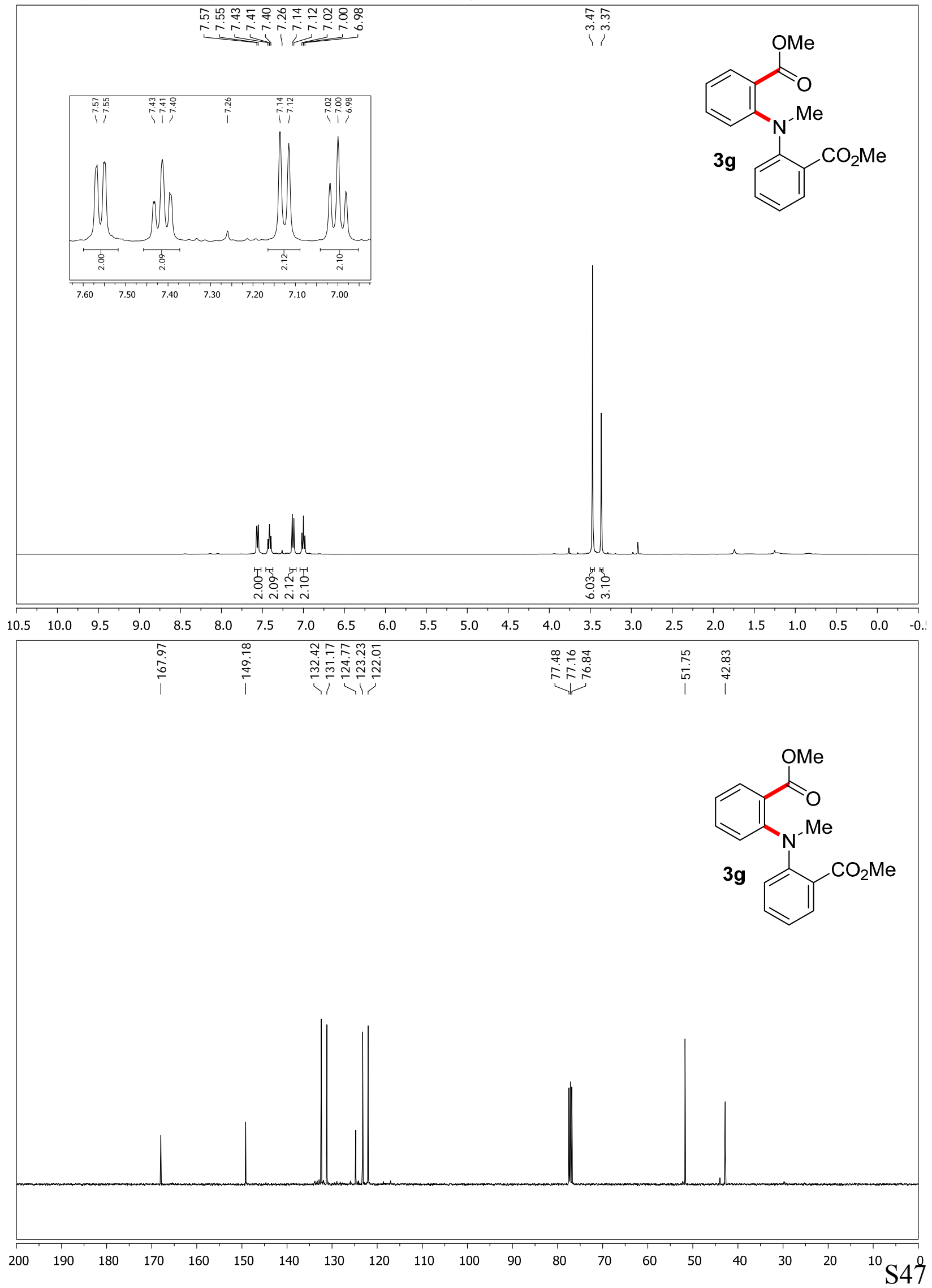


Methyl 2-((3,4-difluorophenyl)(methyl)amino)benzoate (3h)
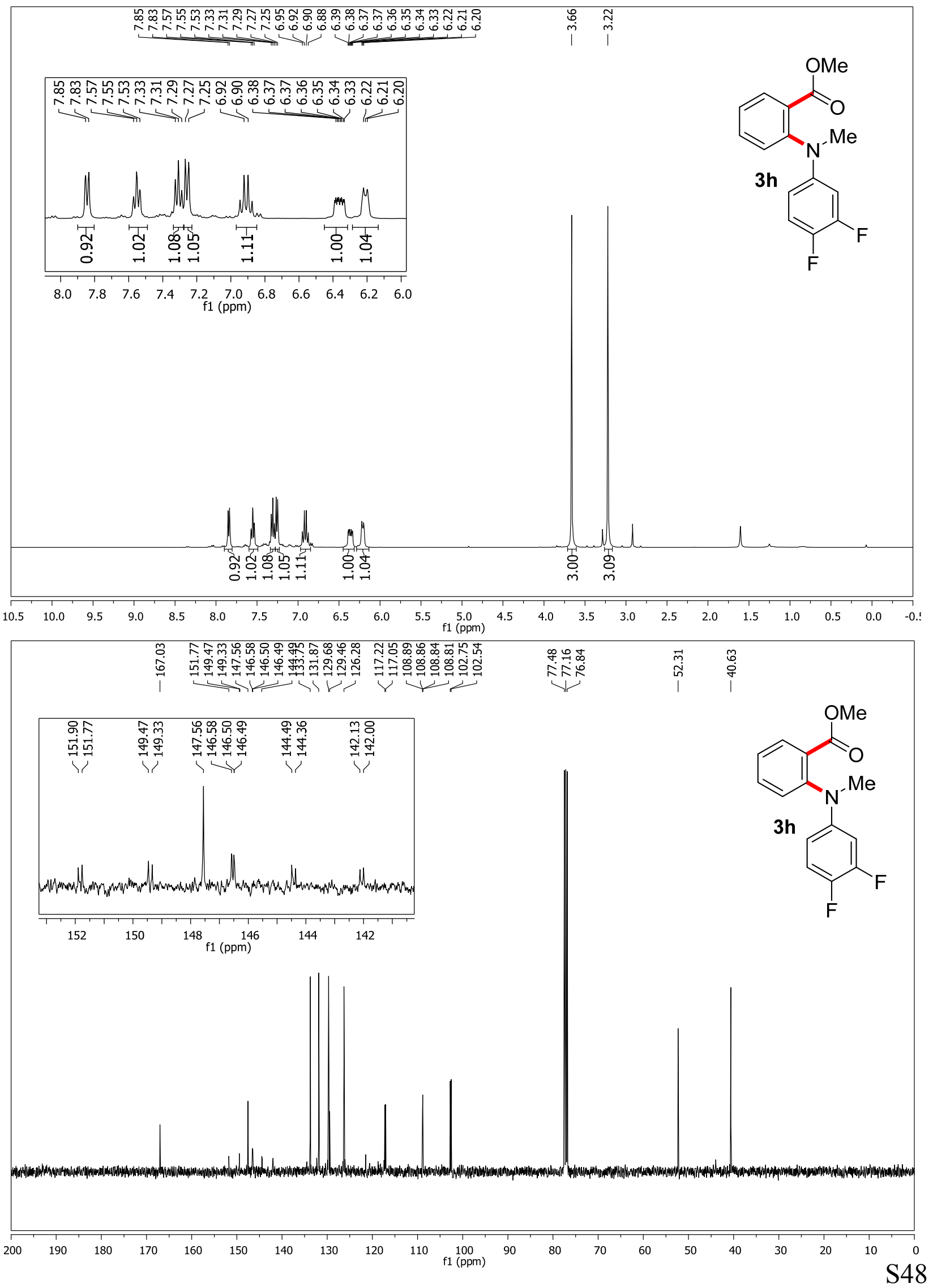
Methyl 2-((3,5-dimethylphenyl)(methyl)amino)benzoate (3i)

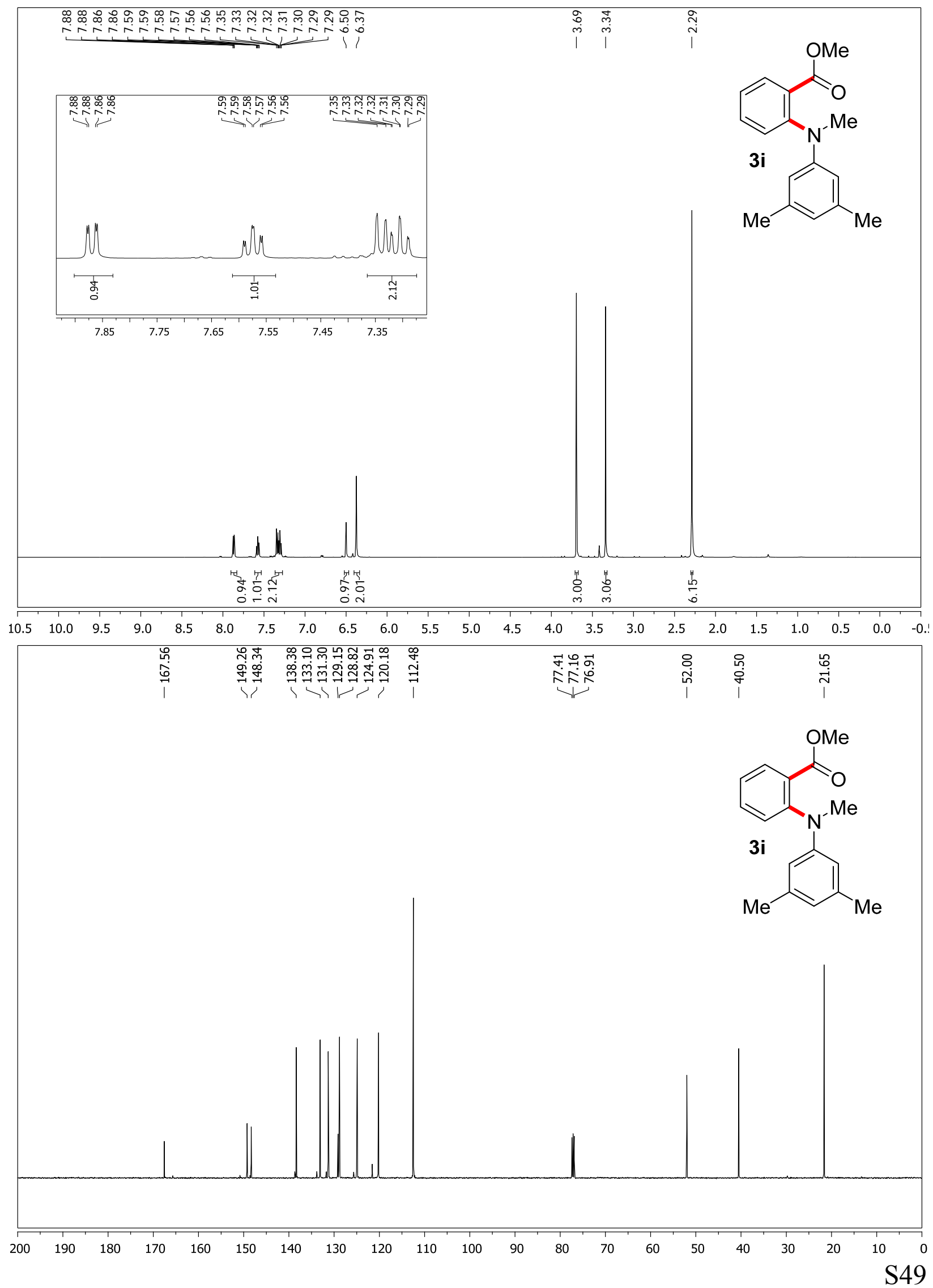


Methyl 2-(methyl(naphthalen-1-yl)amino)benzoate (3j)

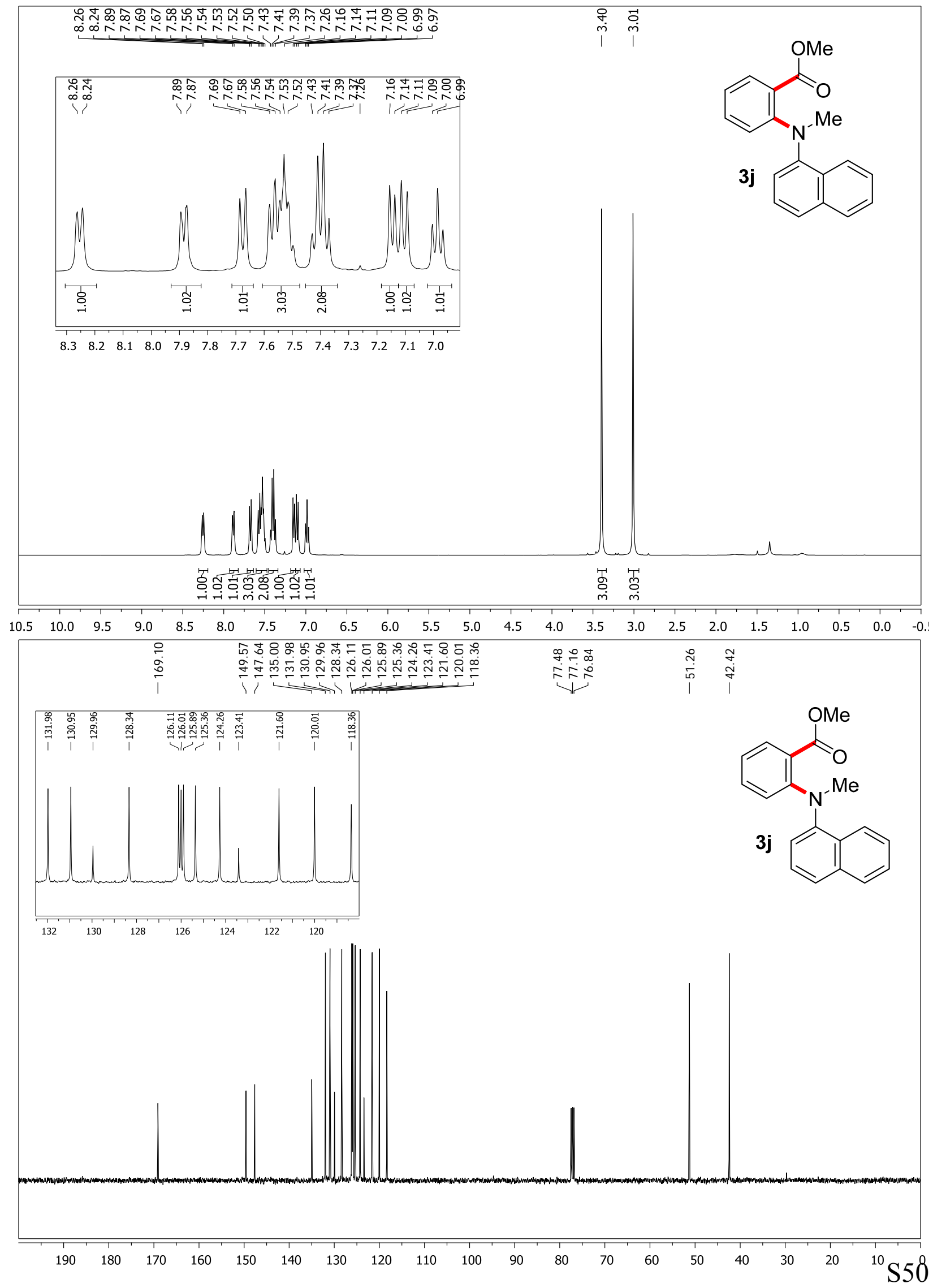


Methyl (E)-2-((4-(2-(5-bromothiophen-2-yl)vinyl)phenyl)(methyl)amino)benzoate (3k)

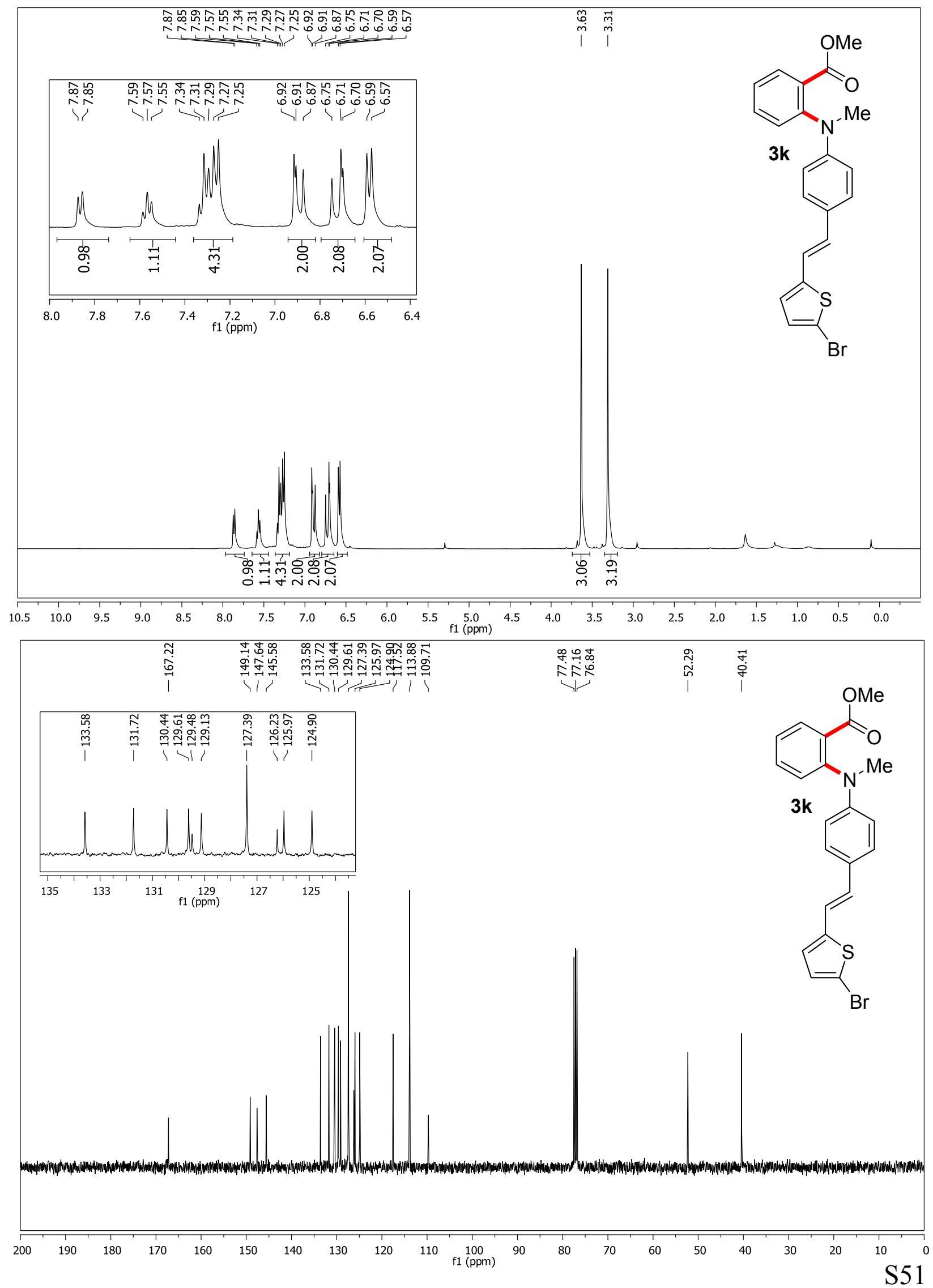


Dimethyl 2,2'-(((phenylmethylene)bis(4,1-phenylene))bis(methylazanediyl))dibenzoate (3I)

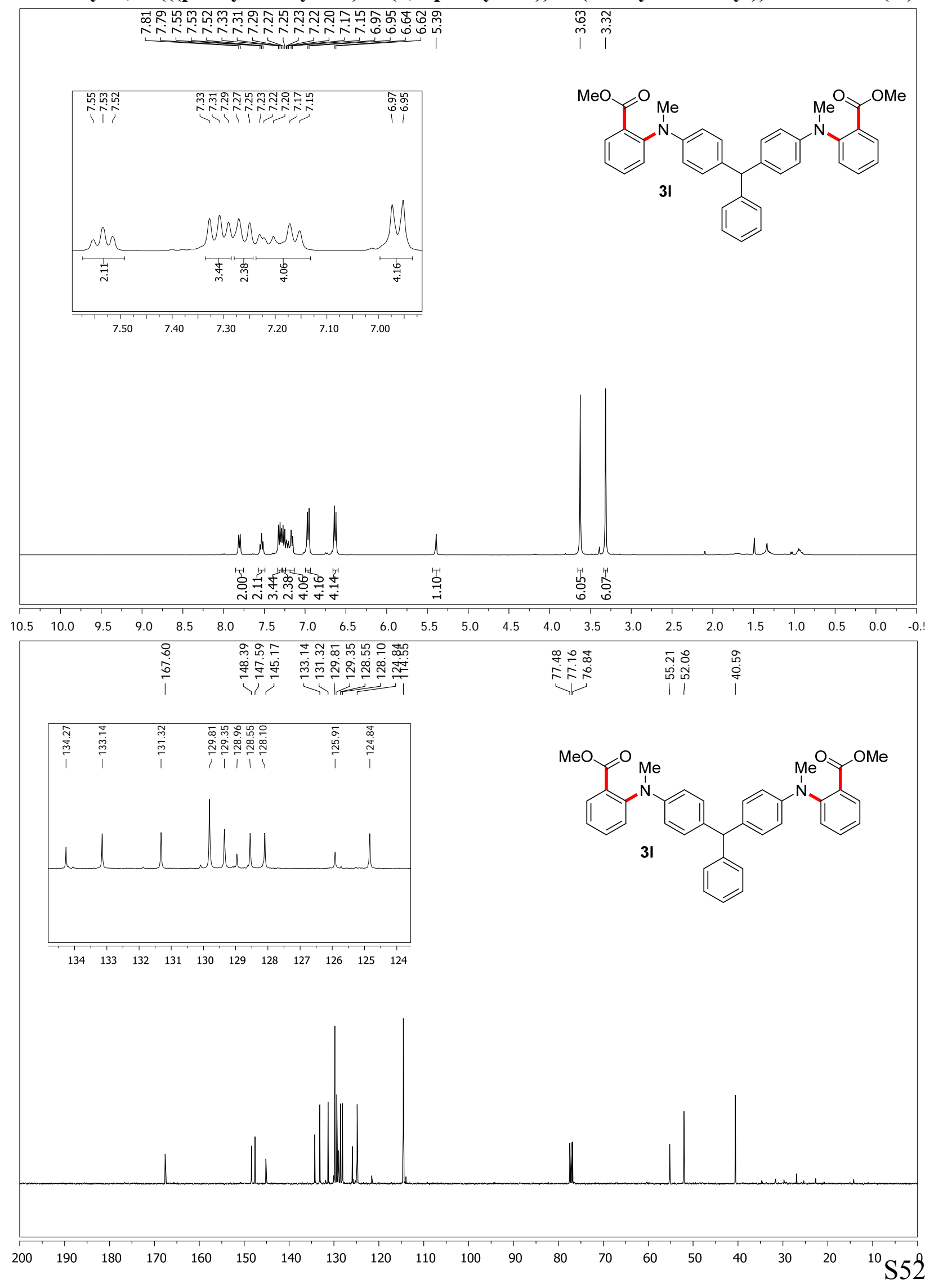


Ethyl 2-(ethyl(phenyl)amino)benzoate (3m)

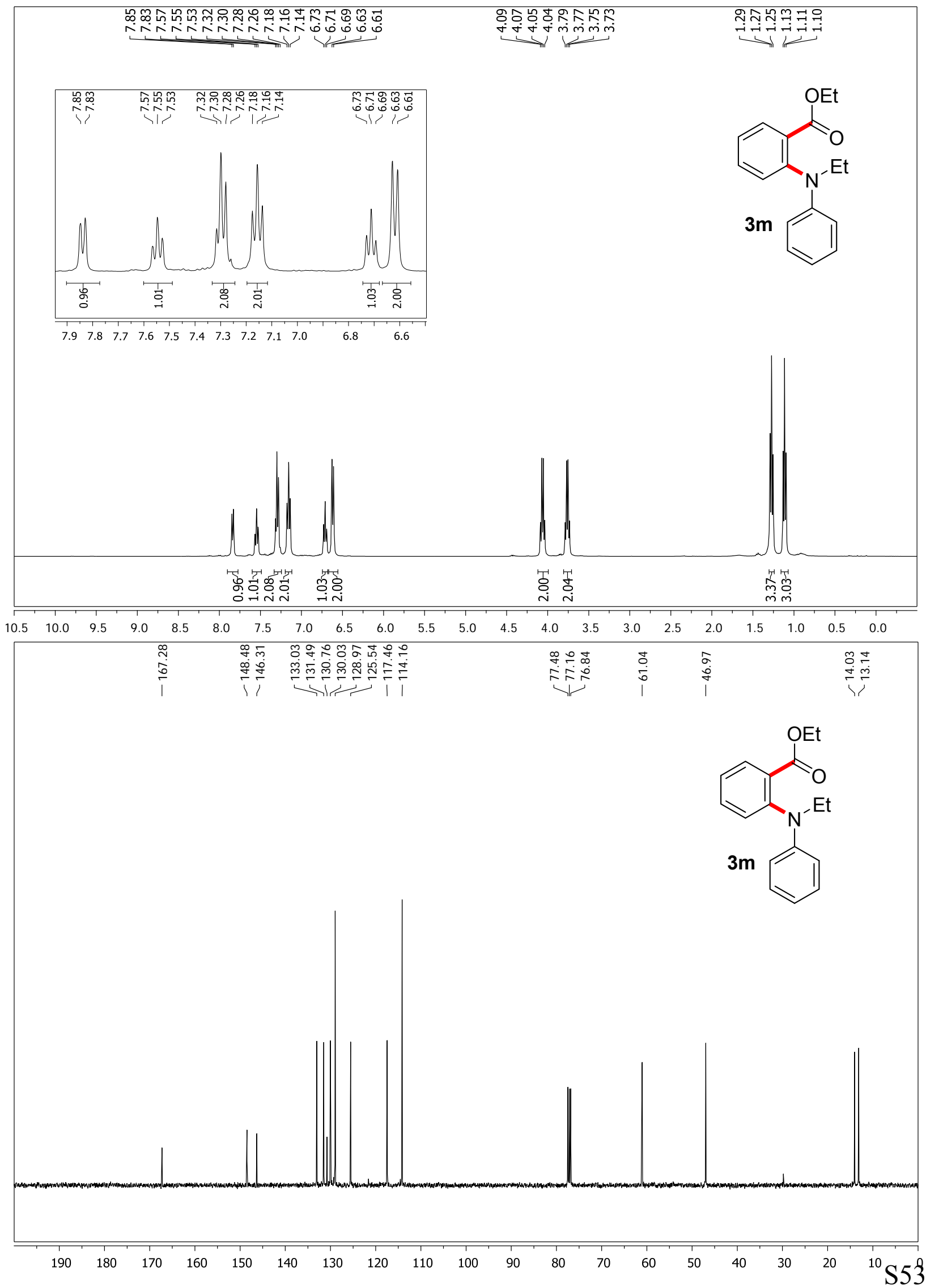




\section{Benzyl 2-(methyl(phenyl)amino)benzoate (3n)}

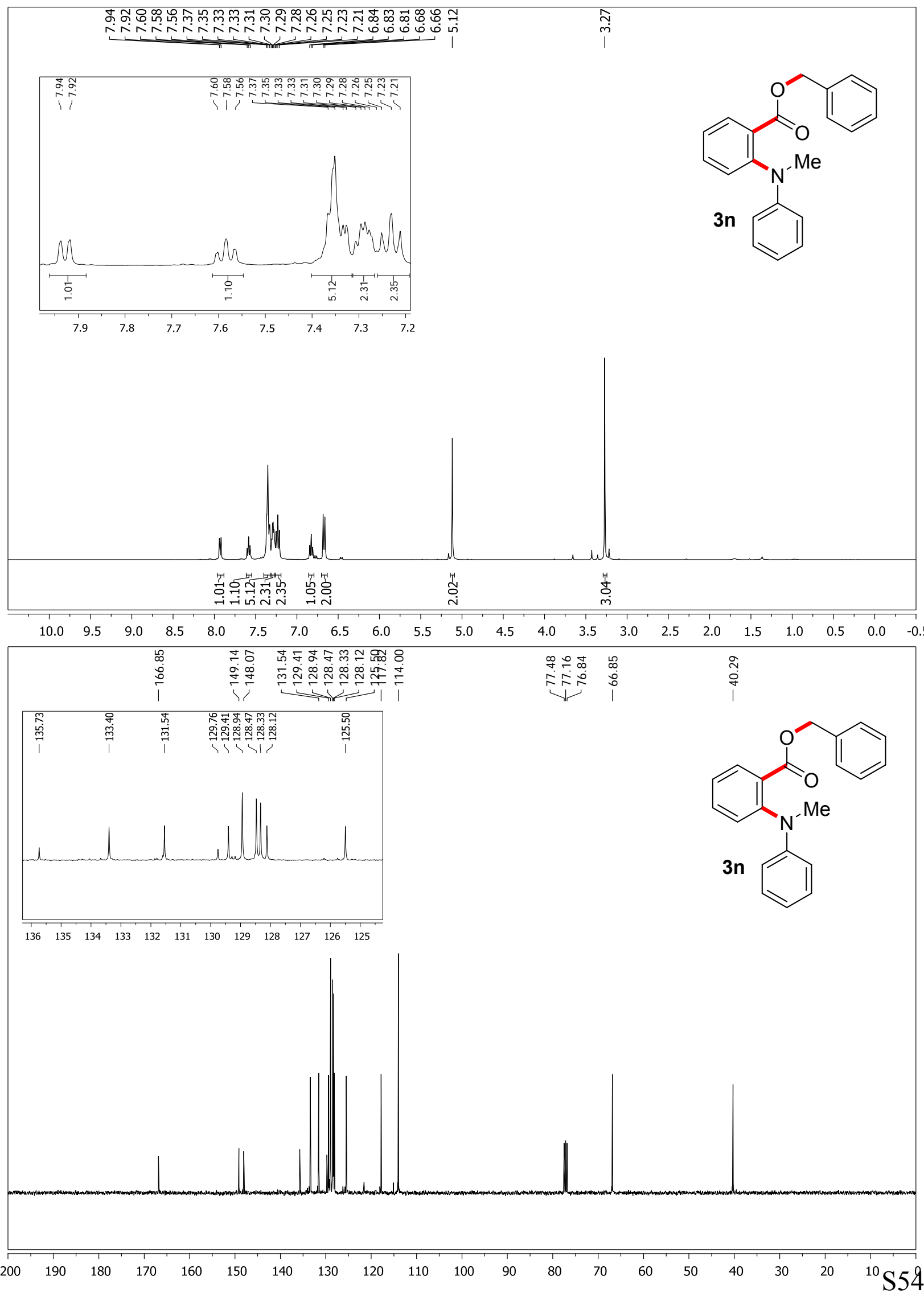


Methyl 4,5-dimethyl-2-(methyl(phenyl)amino)benzoate (3o)

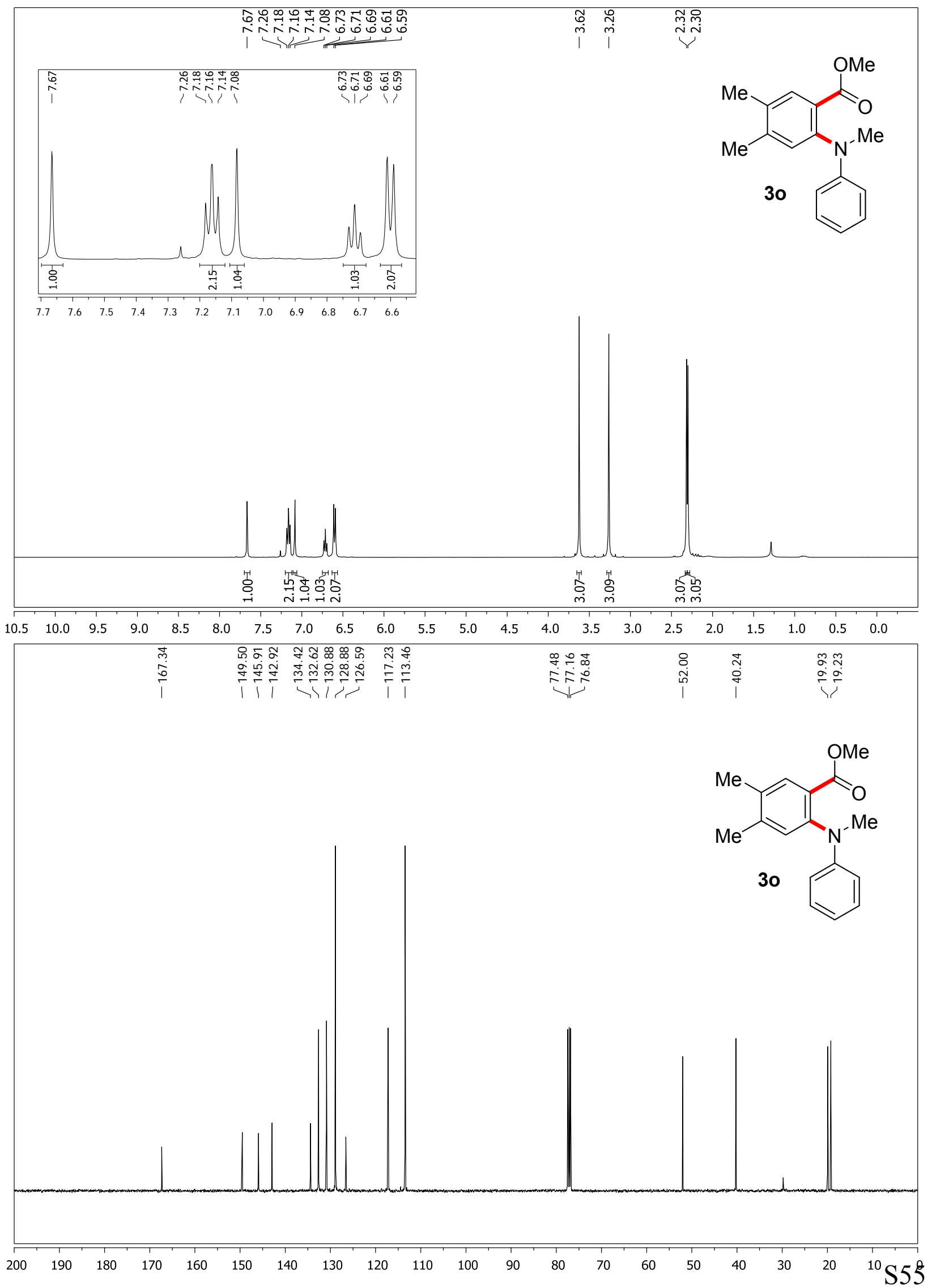


Methyl 6-(methyl(phenyl)amino)benzo[d][1,3]dioxole-5-carboxylate (3p)

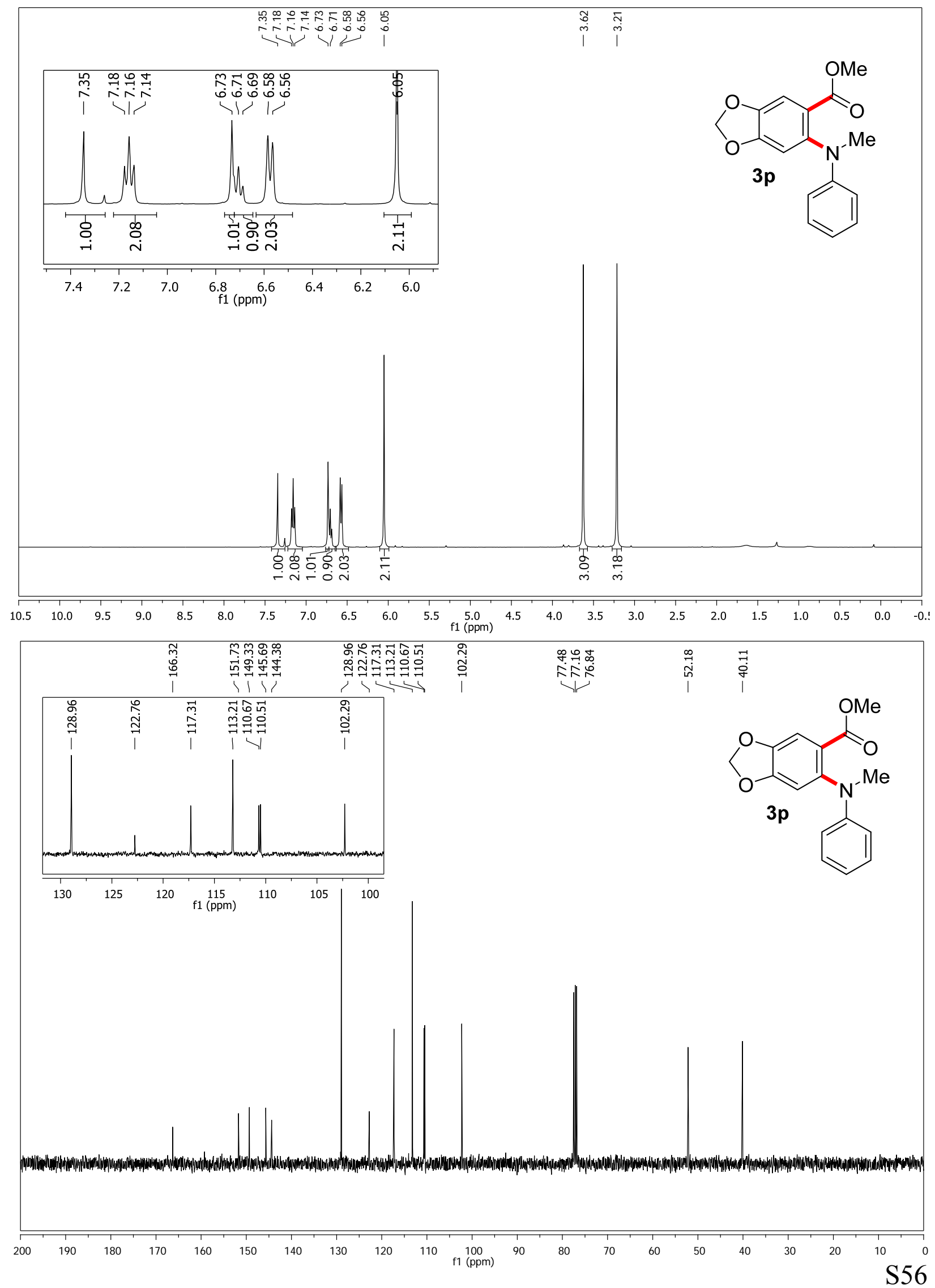


Methyl 4,5-difluoro-2-(methyl(phenyl)amino)benzoate (3q)

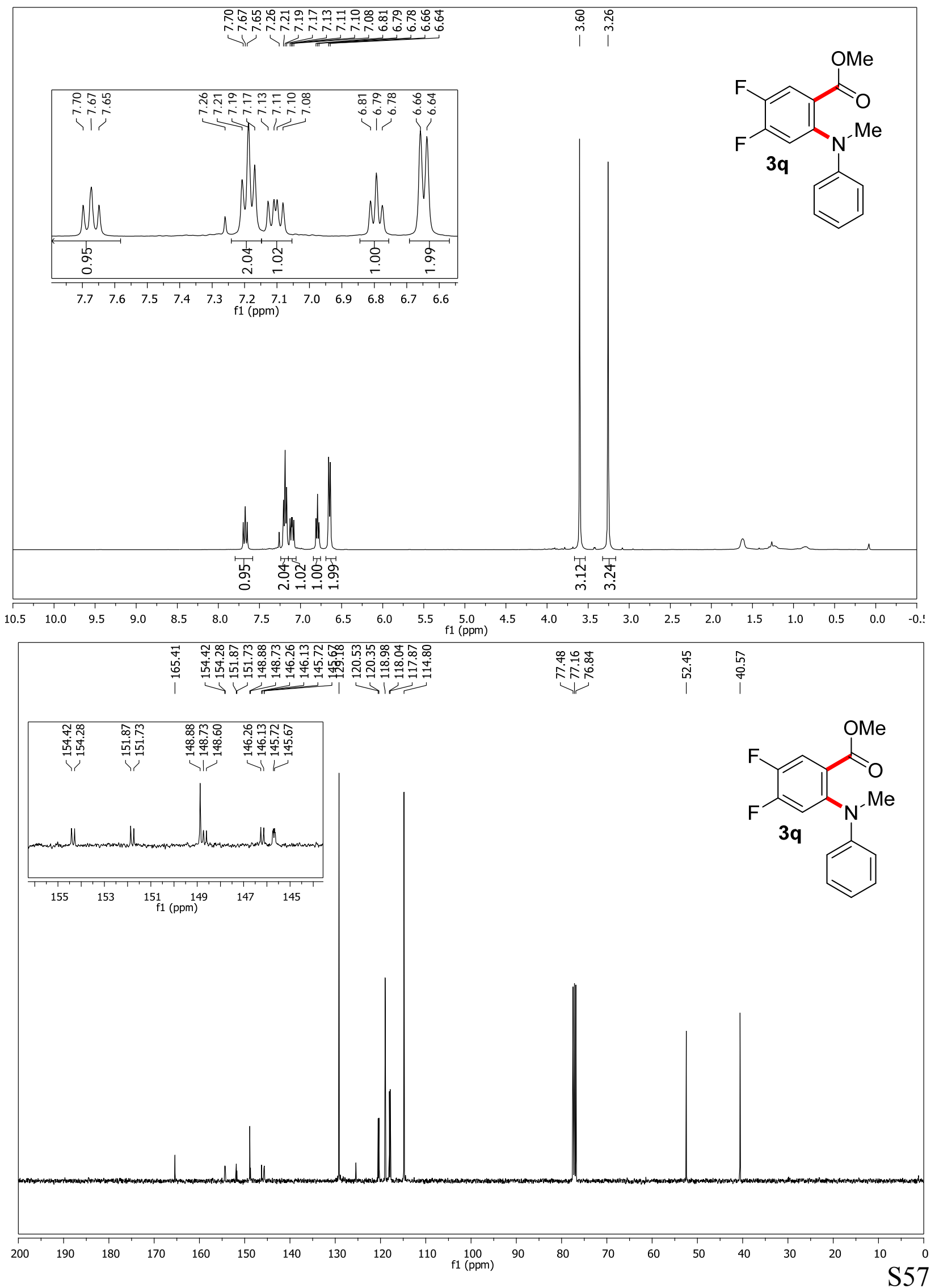


Methyl 3-(methyl(phenyl)amino)-2-naphthoate (3r)
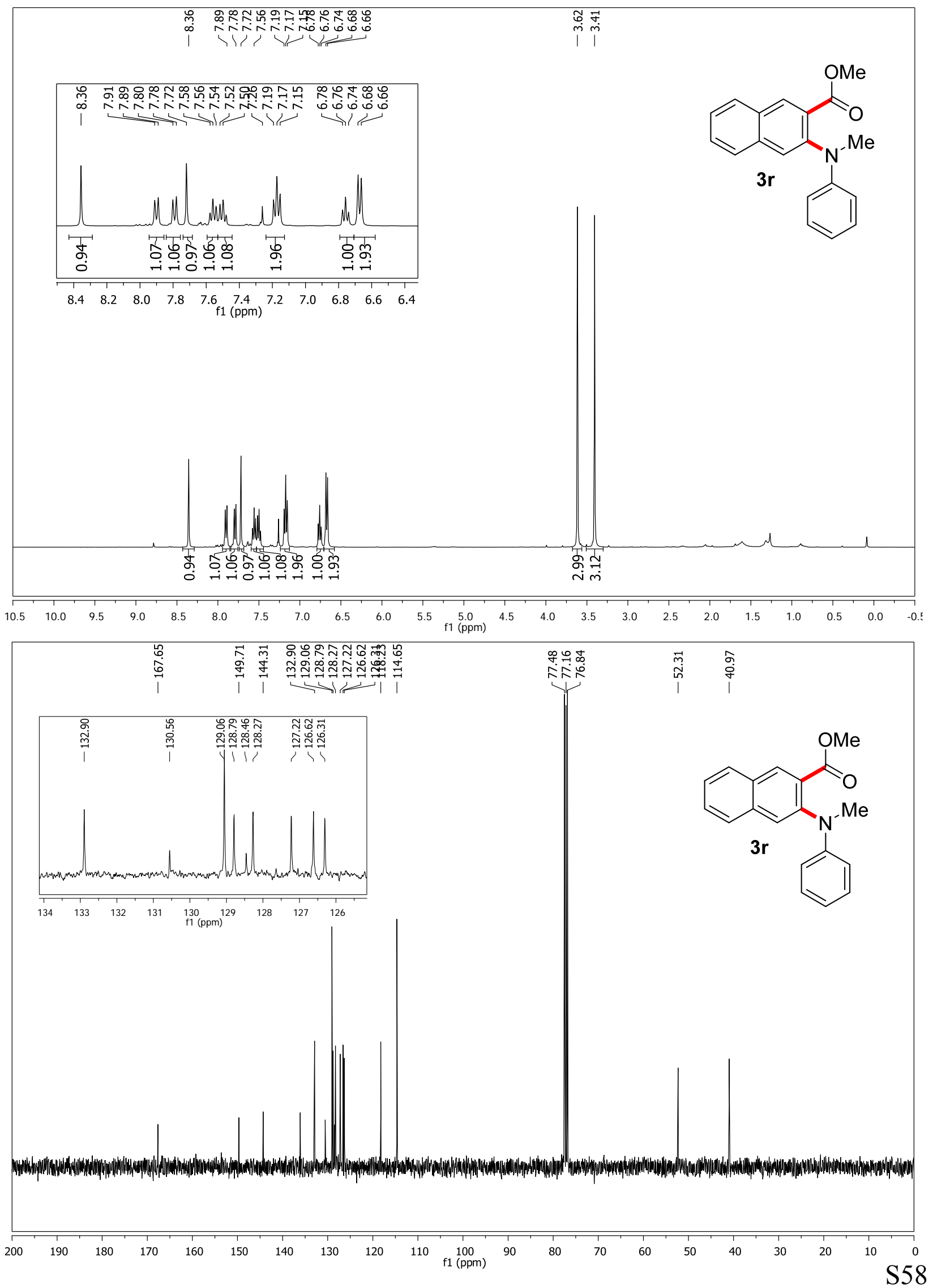
Methyl 2-(methyl(phenyl)amino)-1-naphthoate (3s)
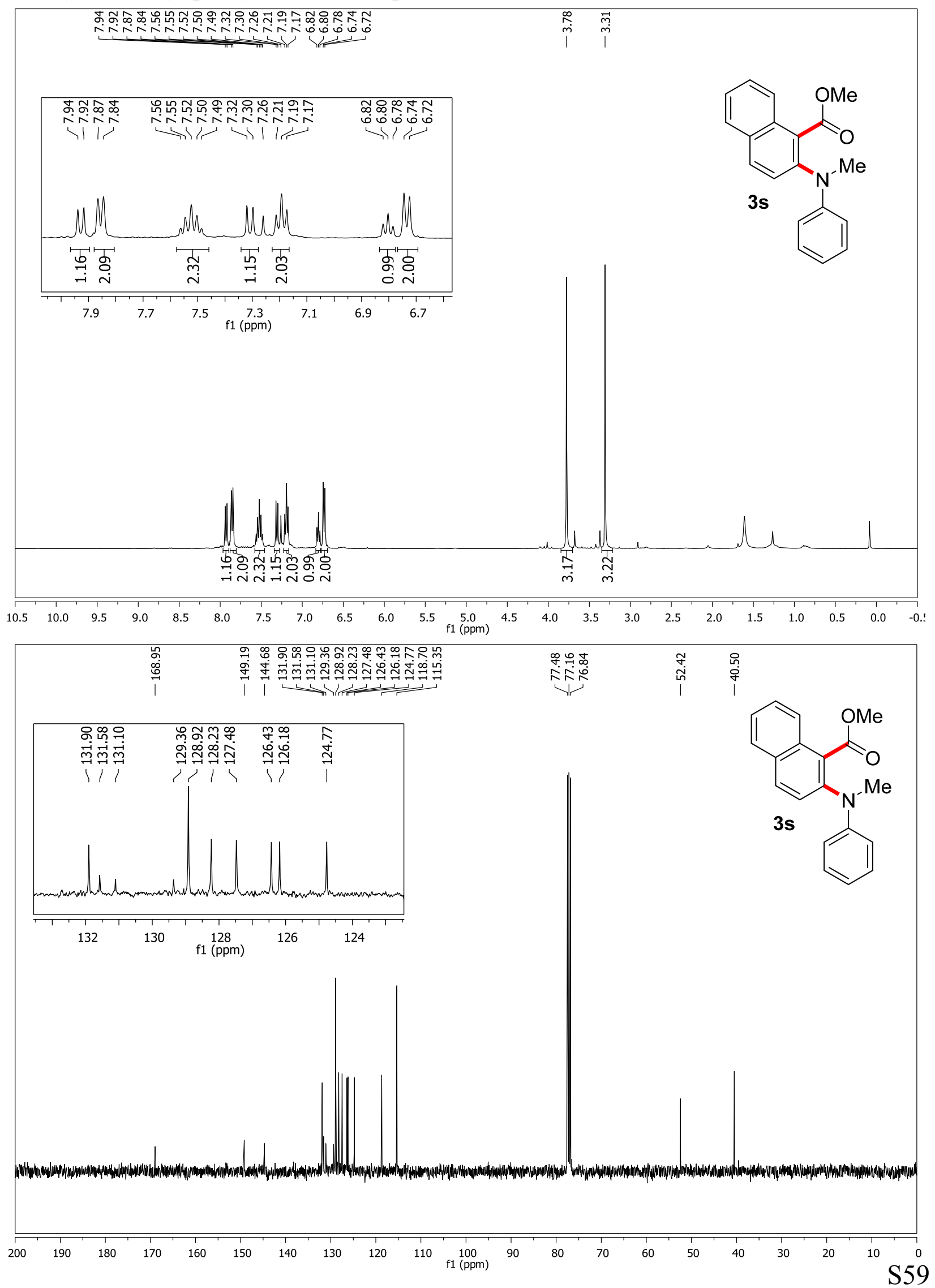
Methyl 5-methyl-2-(methyl(phenyl)amino)benzoate and Methyl 4-methyl-2-(methyl(phenyl)amino)benzoate (3t and 3t')
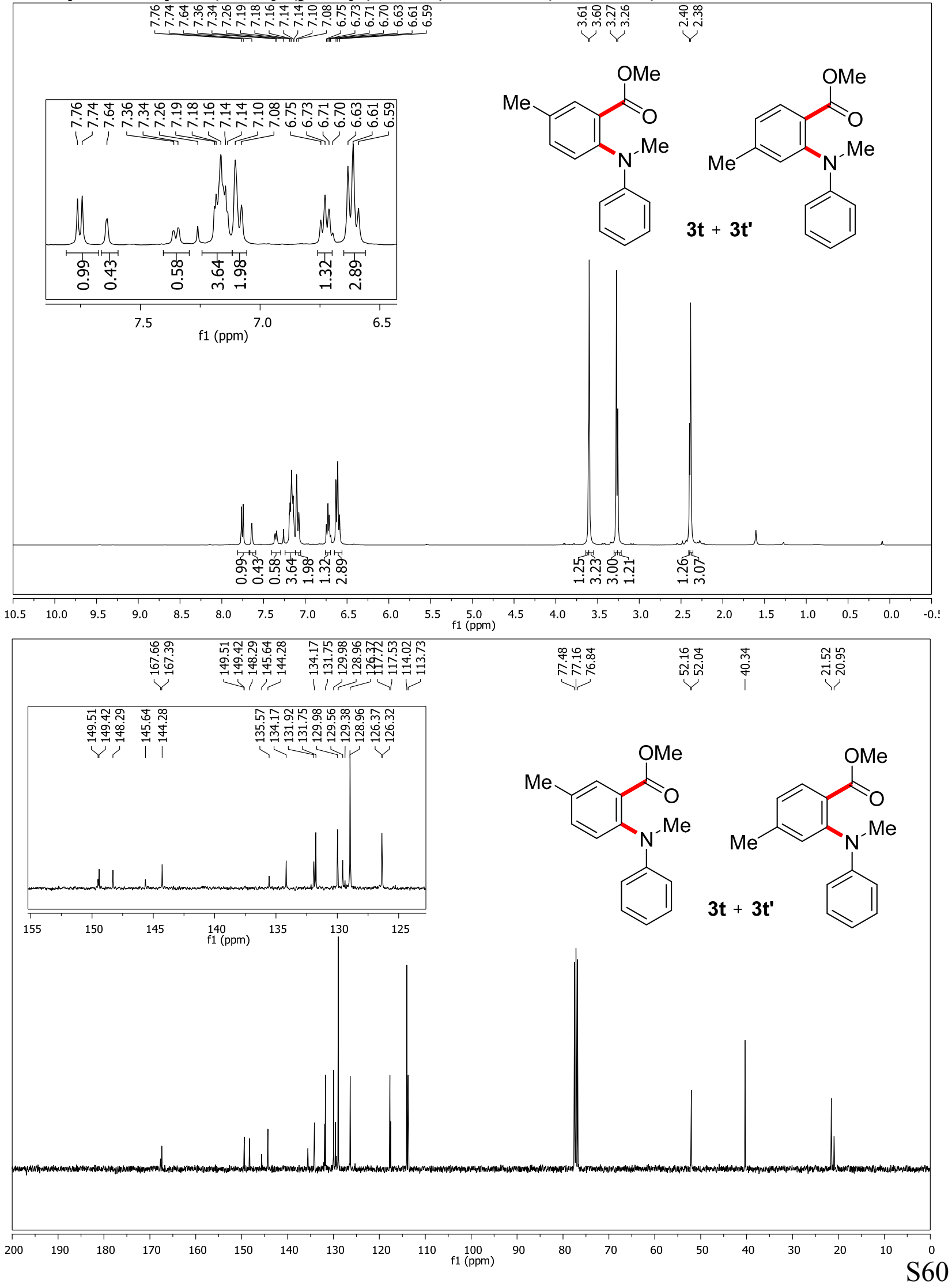
4-(Ethoxycarbonyl)phenyl 2-(dimethylamino)benzoate (4a)

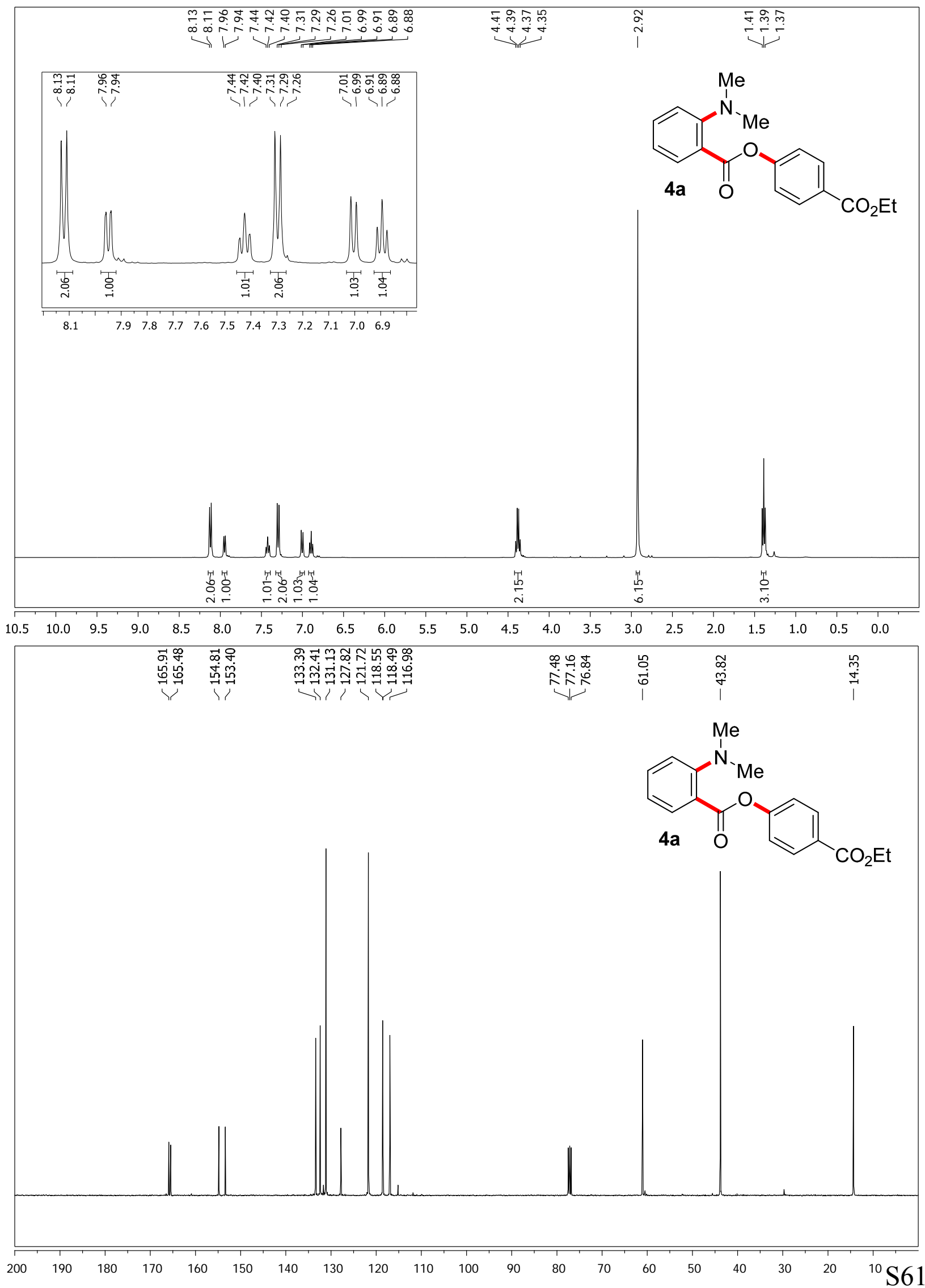




\section{4-(Trifluoromethyl)phenyl 2-(dimethylamino)benzoate (4b)}

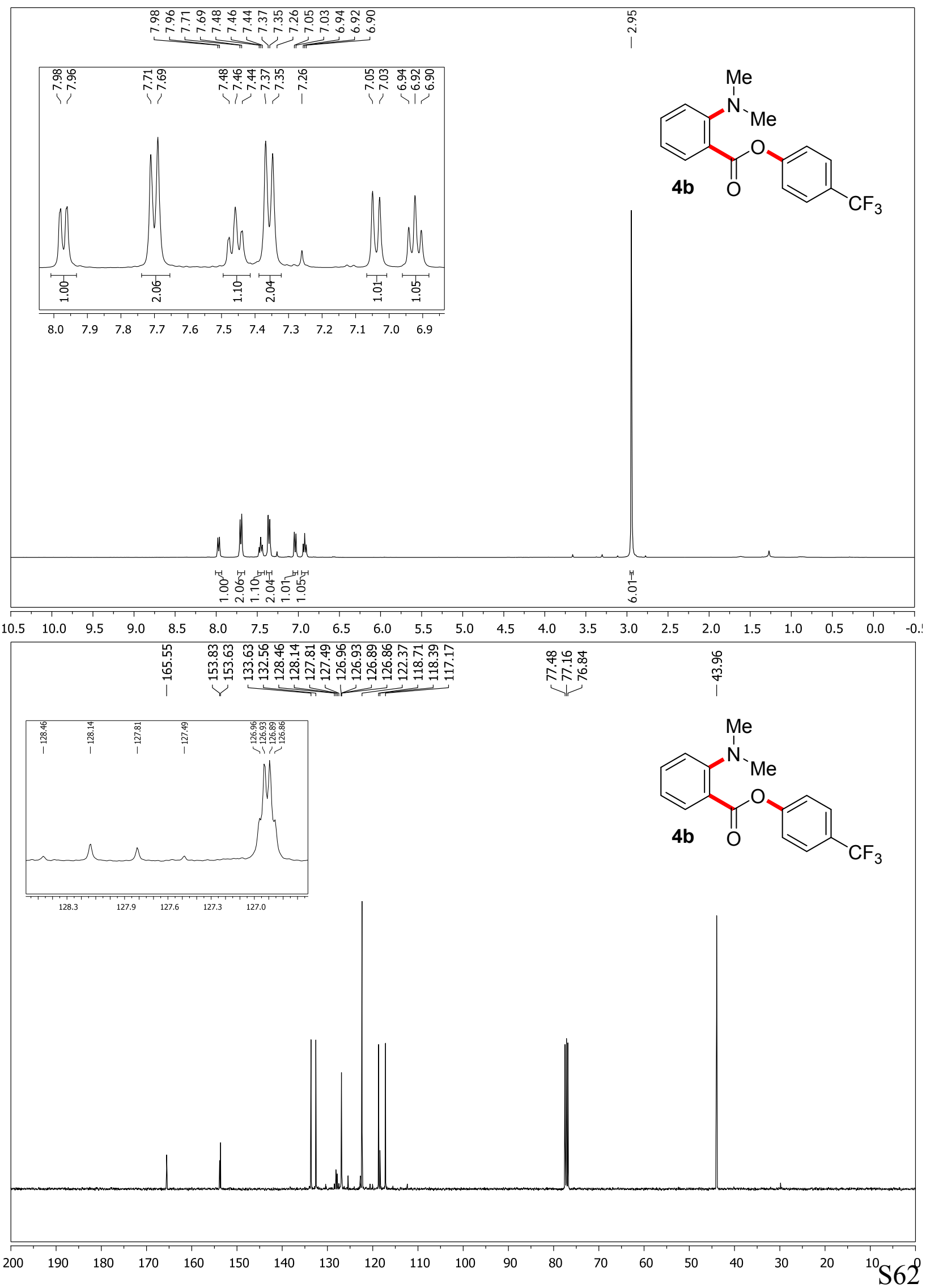


4-Cyanophenyl 2-(dimethylamino)benzoate (4c)

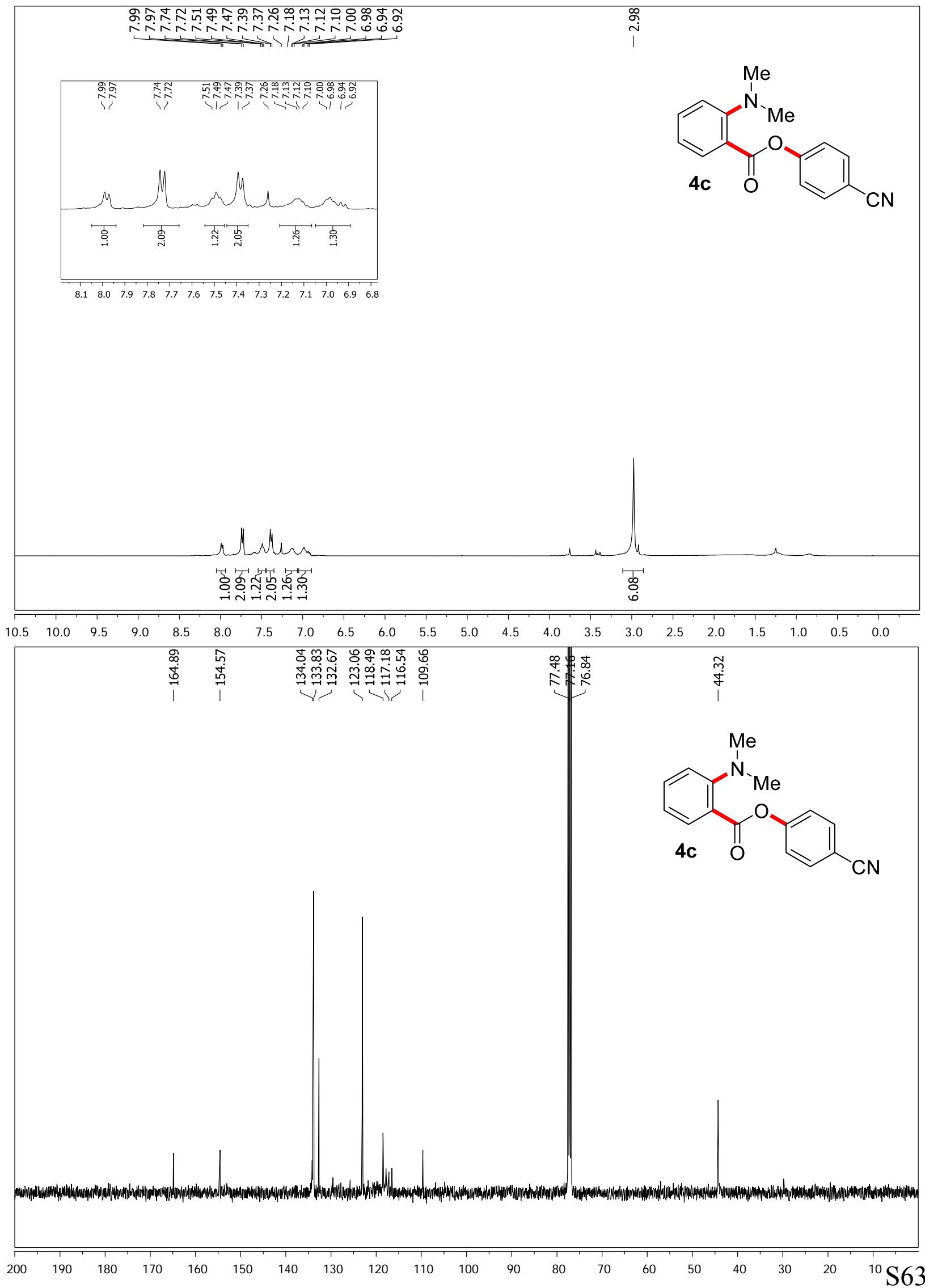


(E)-4-(3-Ethoxy-3-oxoprop-1-en-1-yl)phenyl 2-(dimethylamino)benzoate (4d)

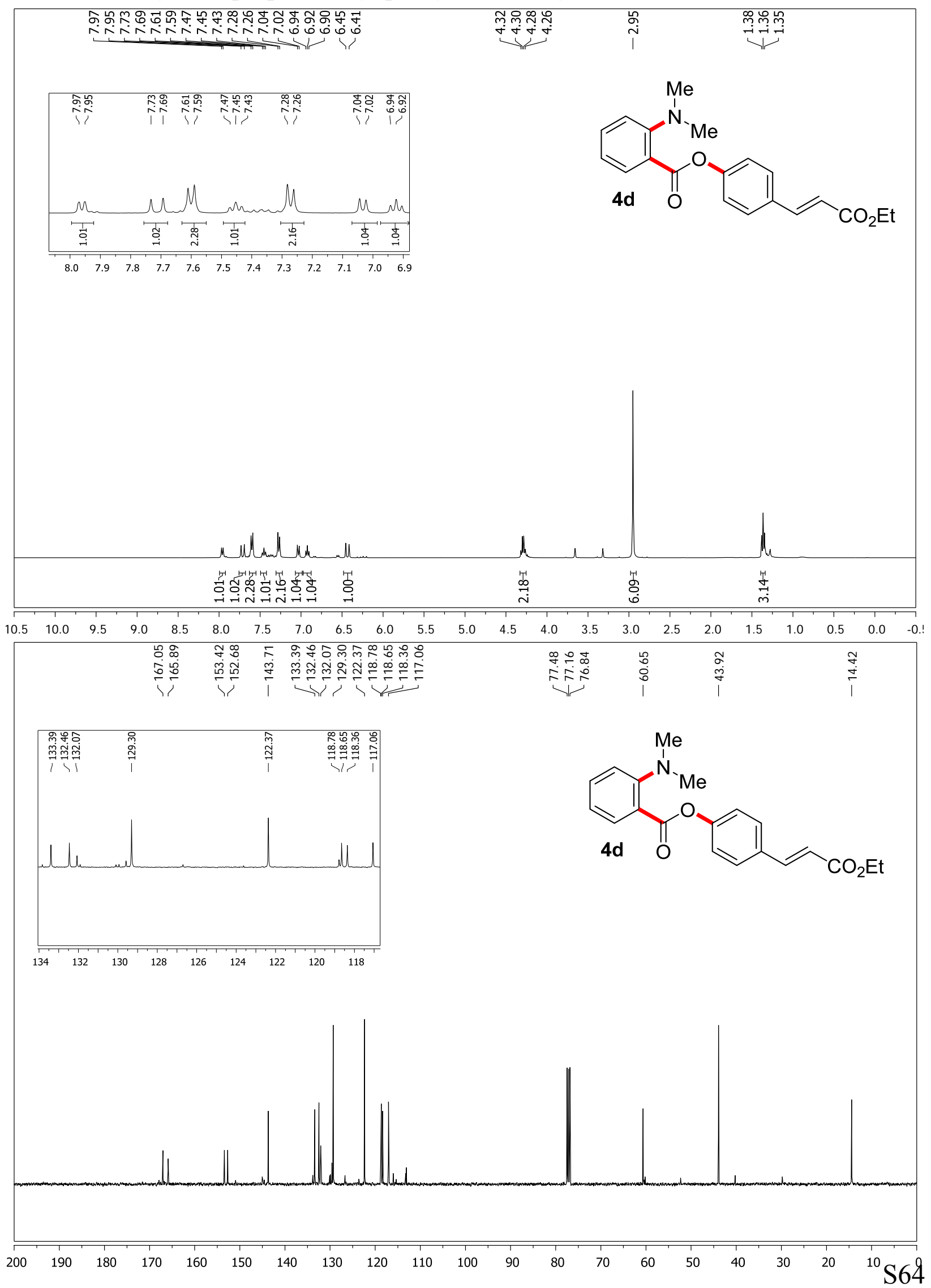


(E)-4-(3-Oxoprop-1-en-1-yl)phenyl 2-(dimethylamino)benzoate (4e)

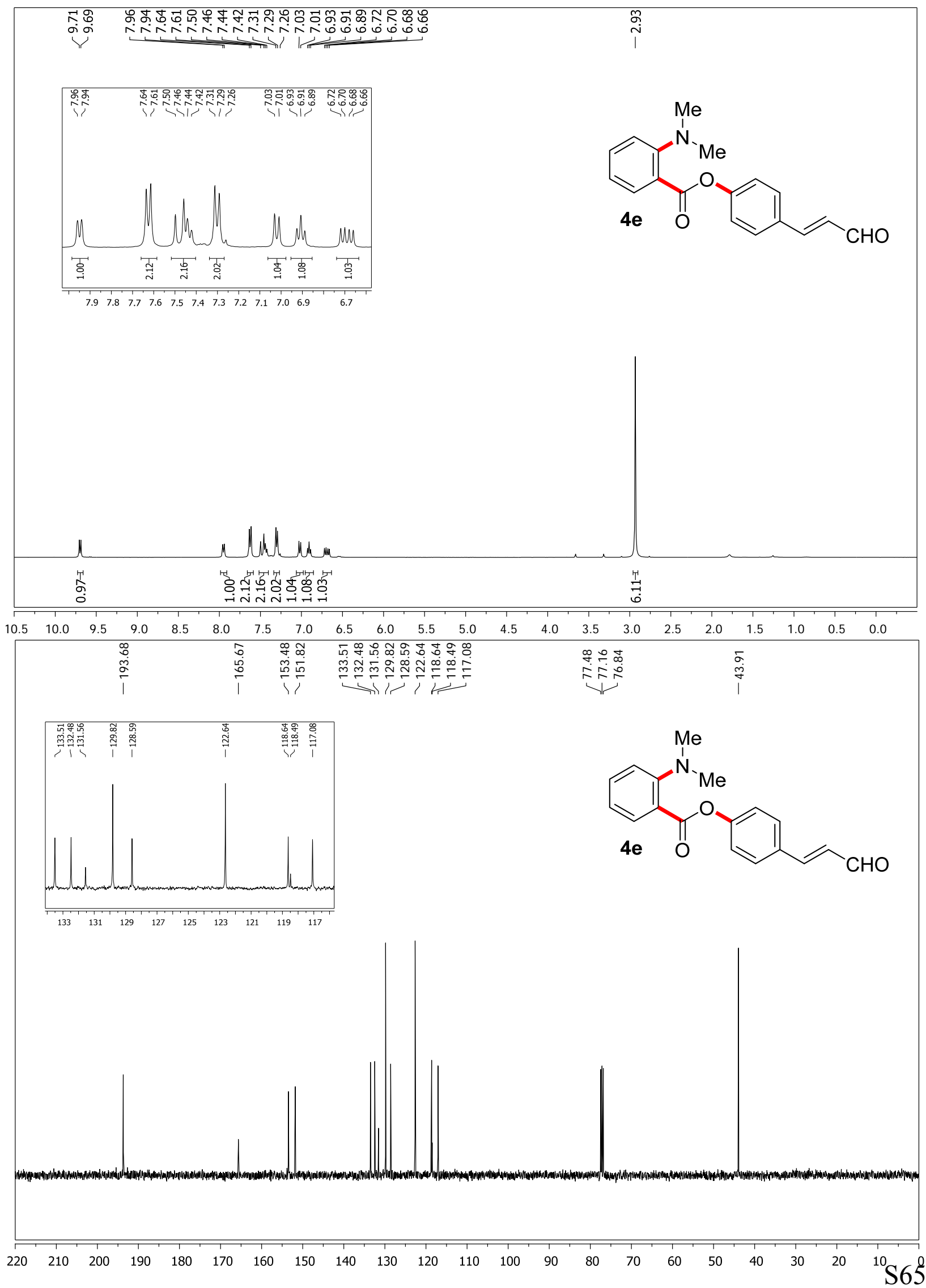


4-(Ethoxycarbonyl)phenyl 2-(dimethylamino)-4,5-dimethylbenzoate (4f)

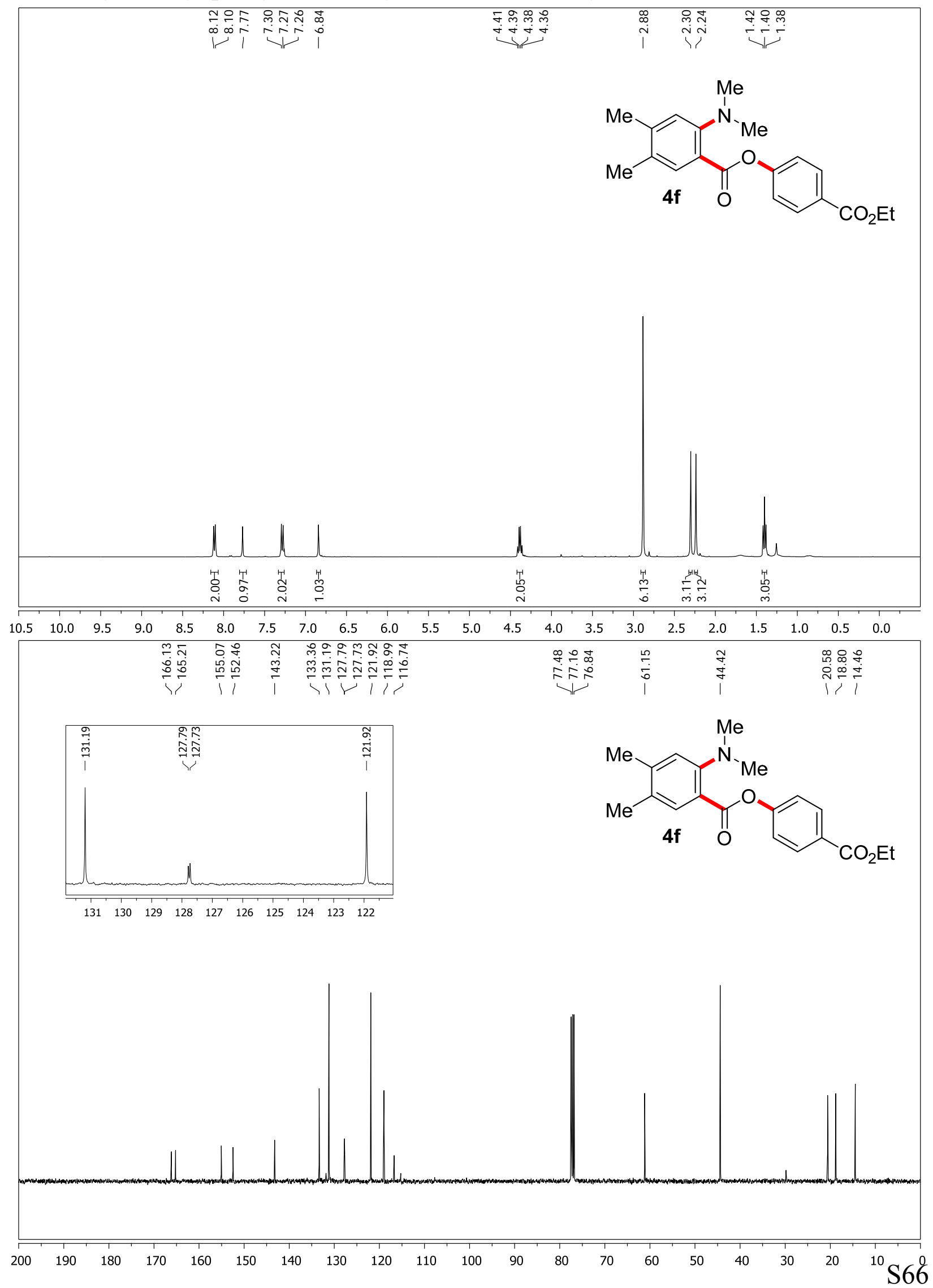


4-(Ethoxycarbonyl)phenyl 6-(dimethylamino)benzo $[d][1,3]$ dioxole-5-carboxylate (4g)

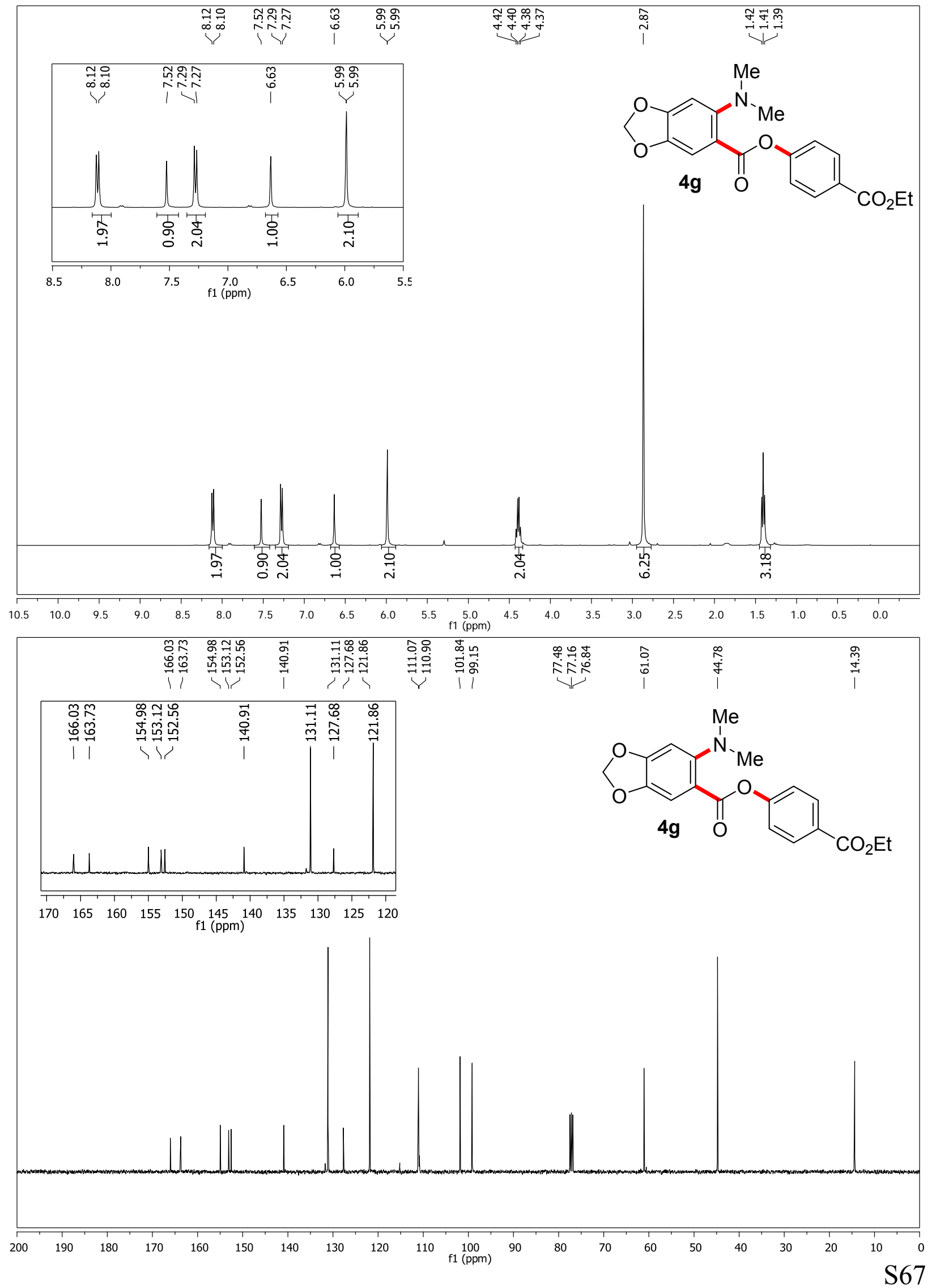


4-(Ethoxycarbonyl)phenyl 2-(dimethylamino)-4,5-difluorobenzoate (4h)

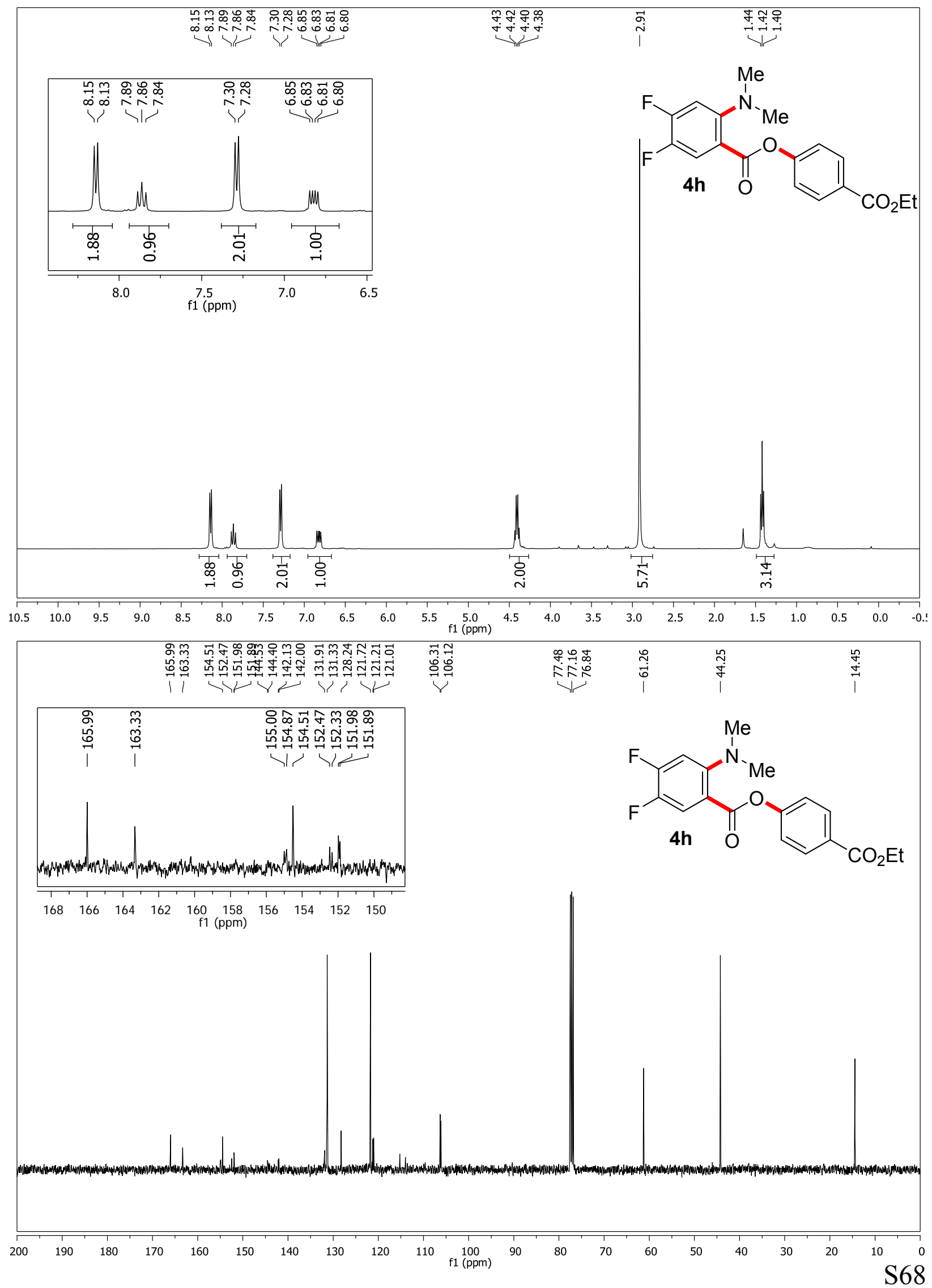


4-(Ethoxycarbonyl)phenyl 3-(dimethylamino)-2-naphthoate (4i)

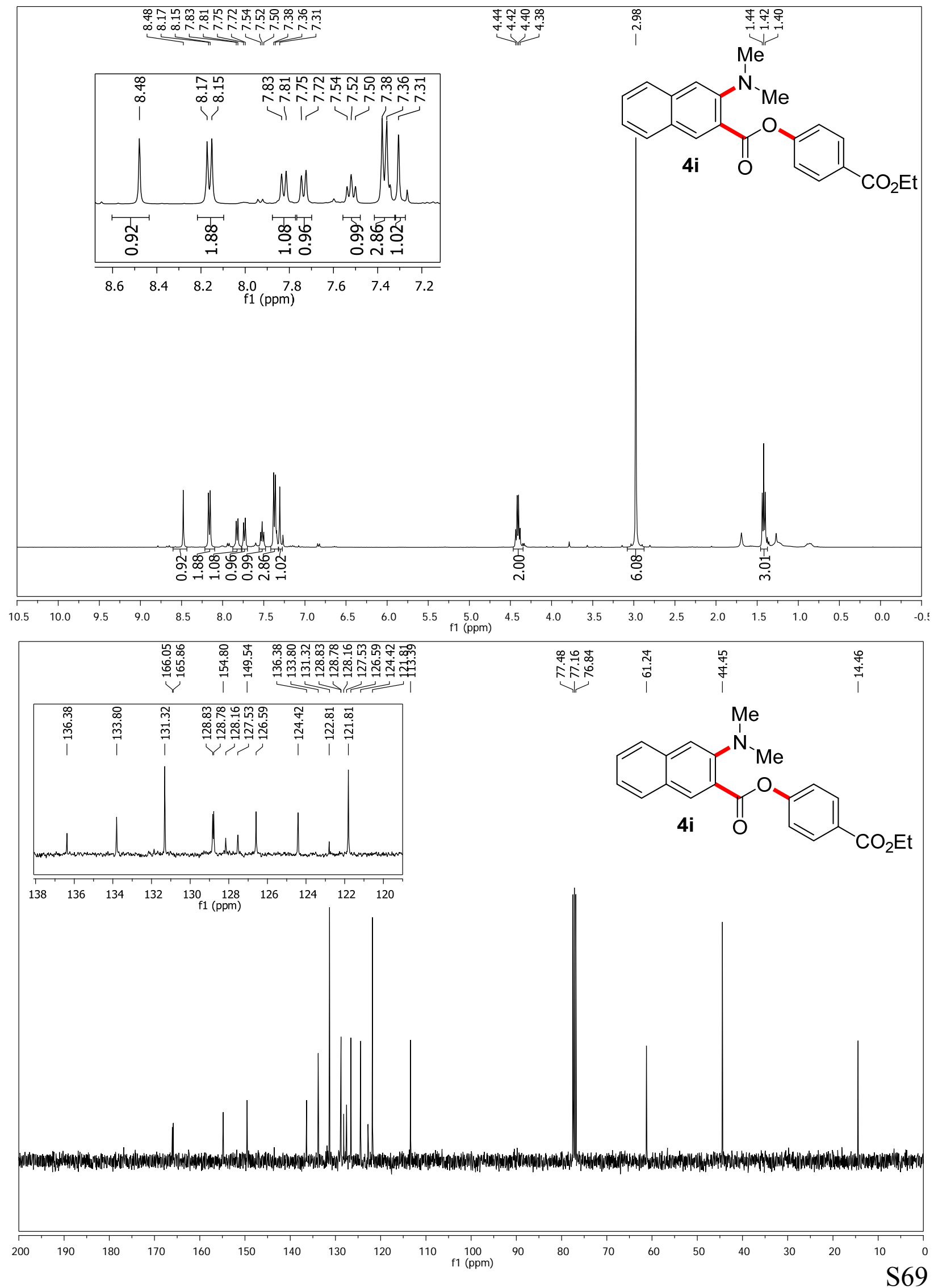


4-(Ethoxycarbonyl)phenyl 2-(dimethylamino)-1-naphthoate (4j)
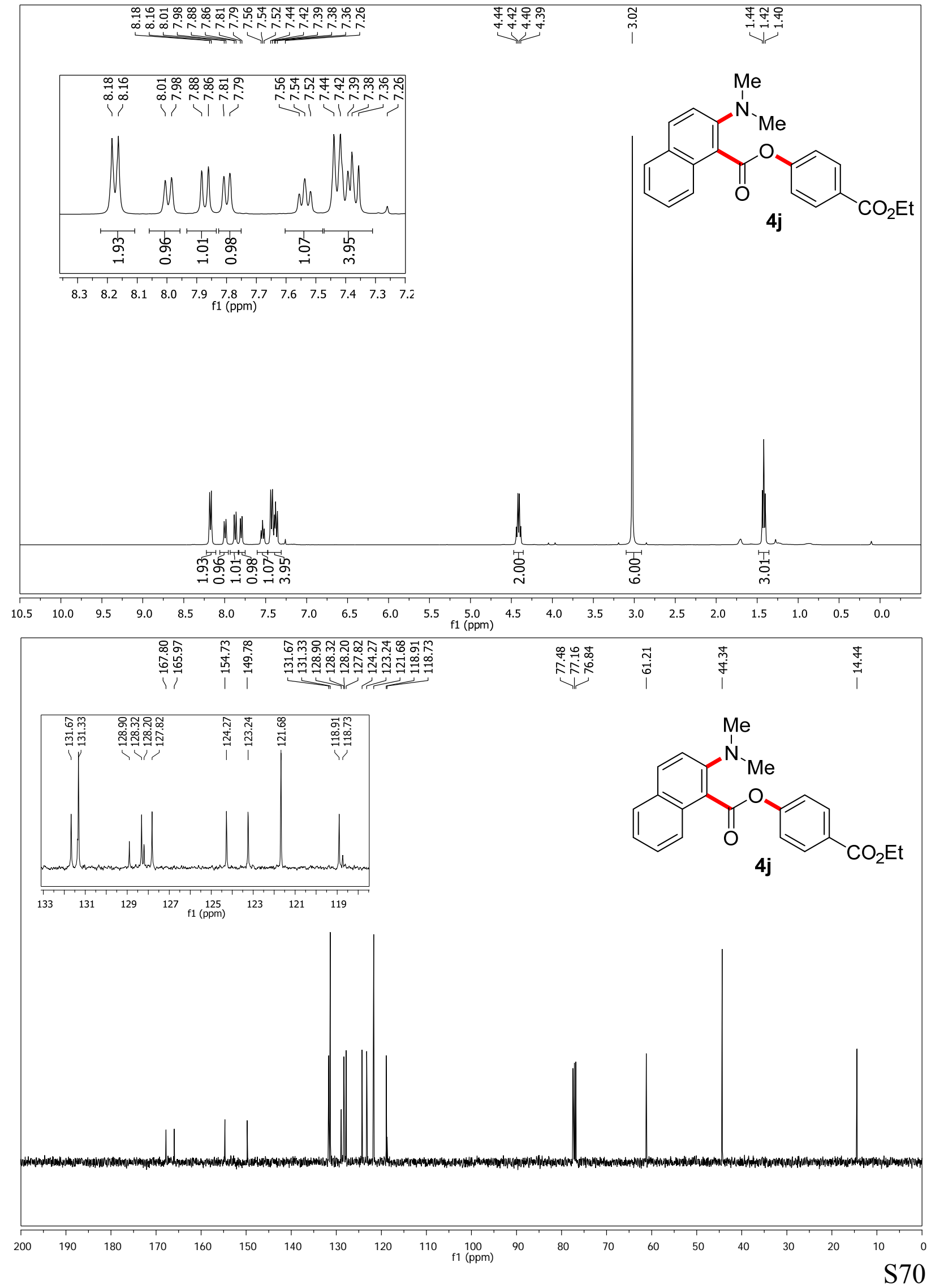
4-(Ethoxycarbonyl)phenyl 2-(dimethylamino)-5-methylbenzoate and 4-(Ethoxycarbonyl)phenyl 2-(dimethylamino)-4-methylbenzoate (4k and 4k')

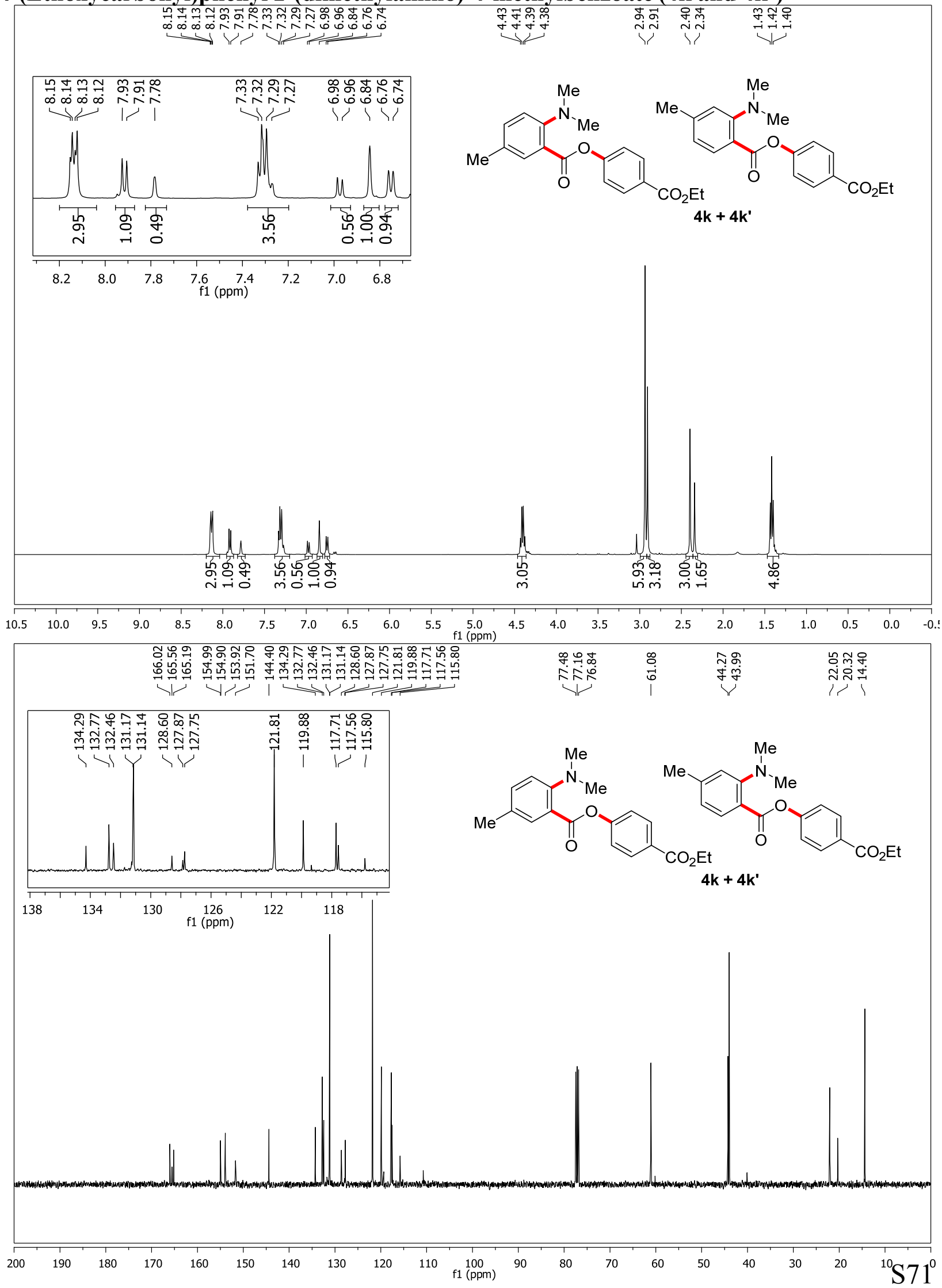


2-(Methyl(phenyl)amino)benzaldehyde (9)

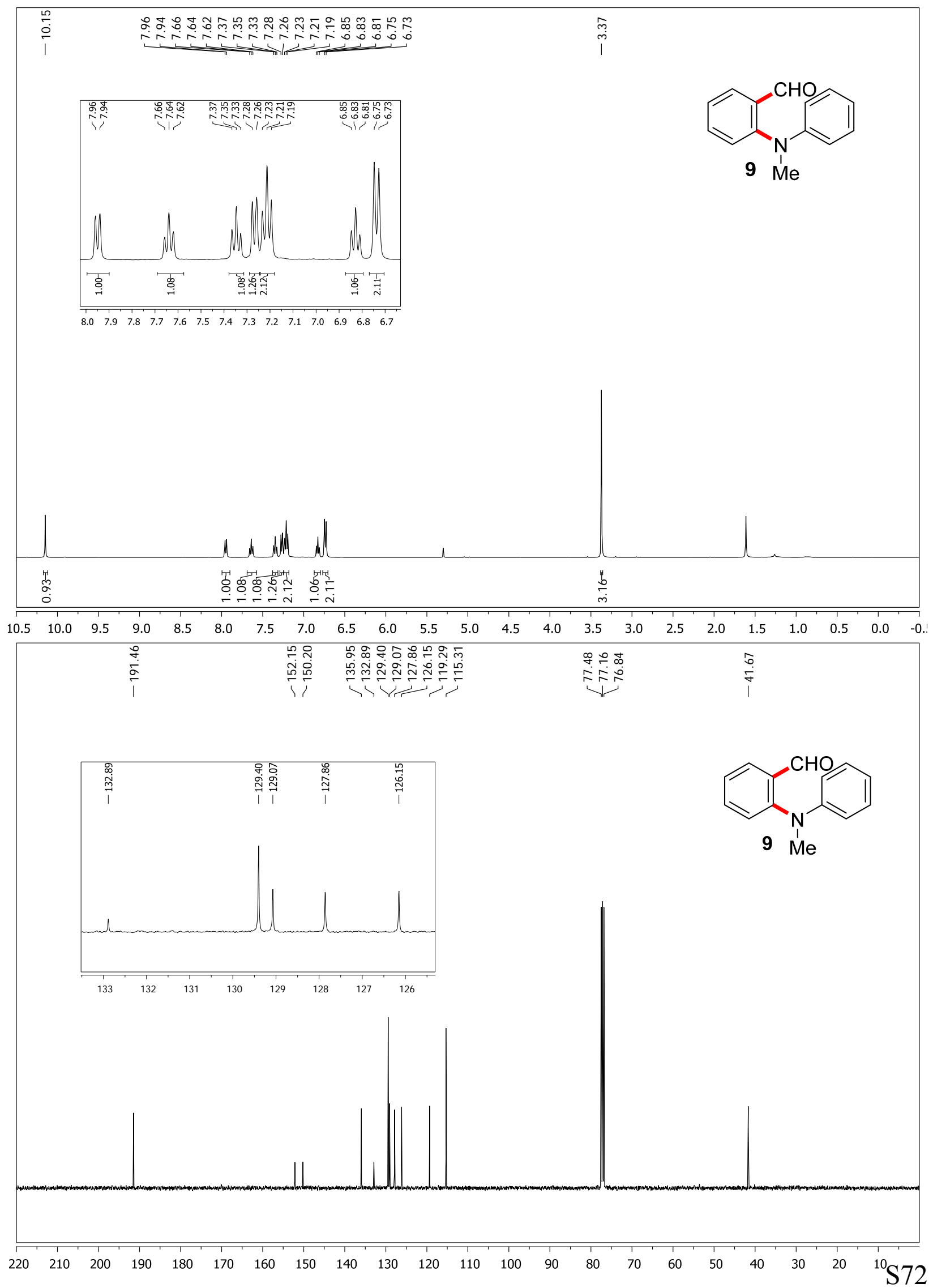


10-Methylacridin-9(10H)-one (10)

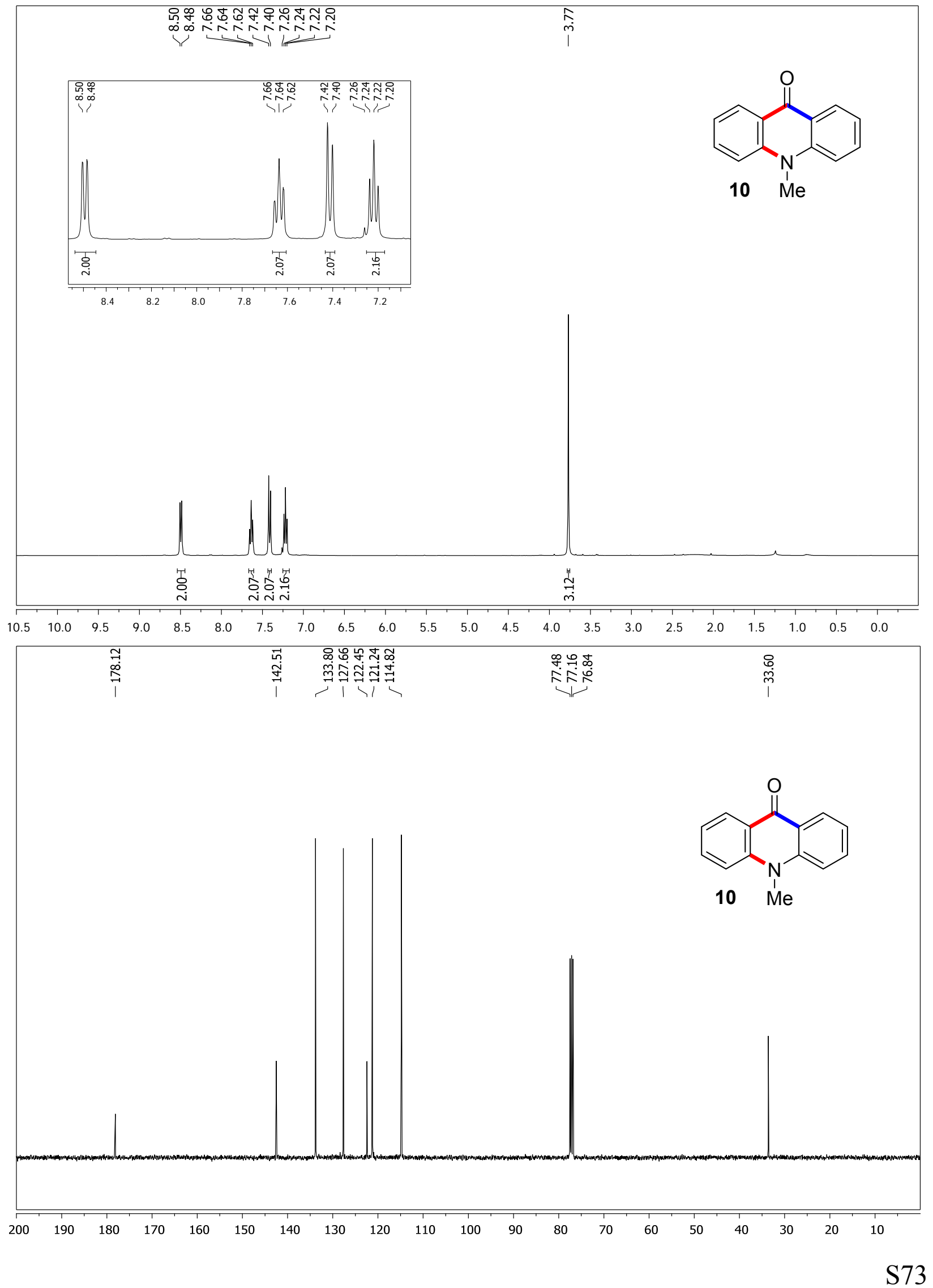




\section{2-(Methyl(phenyl)amino)benzoic acid (11)}

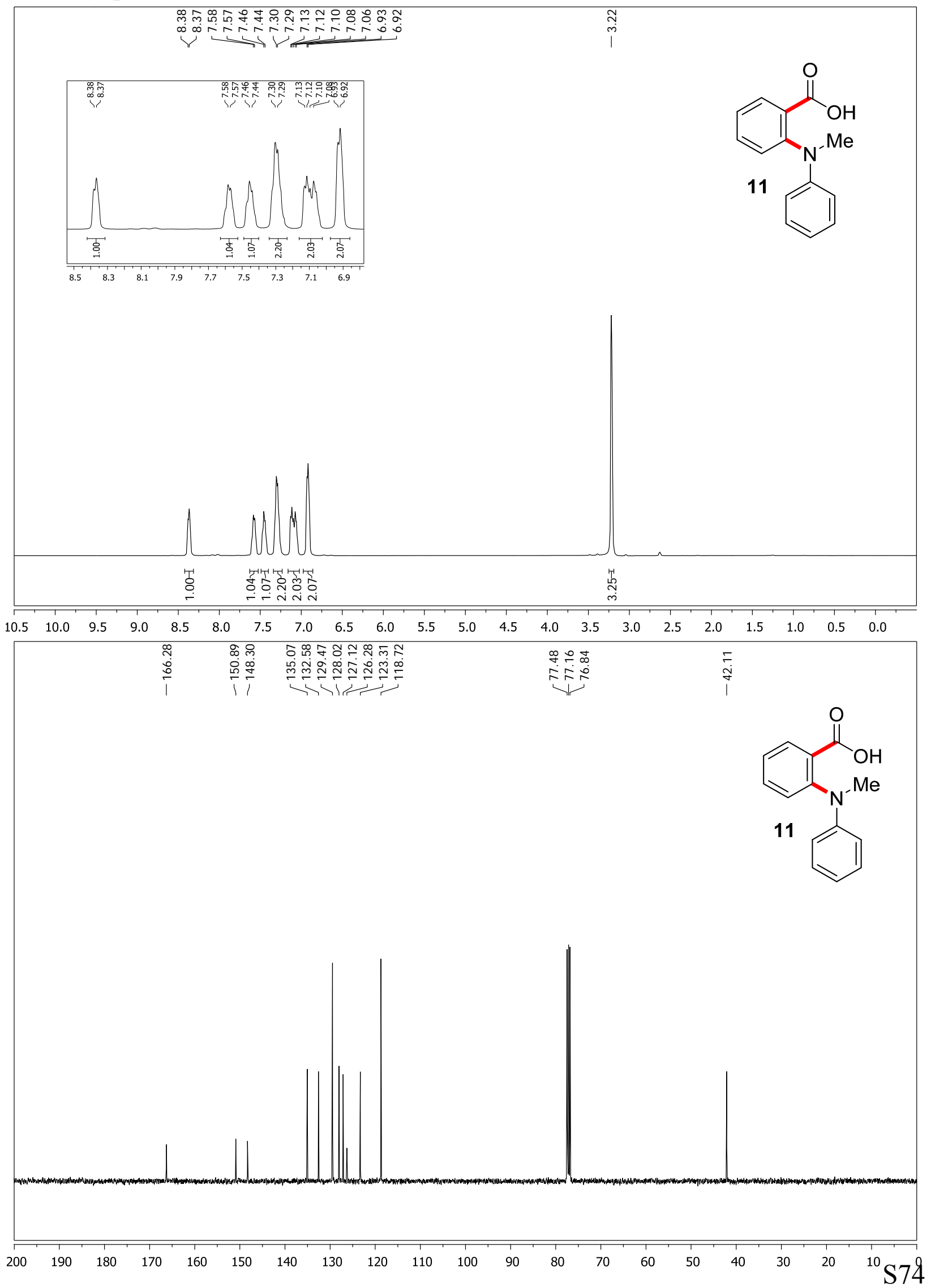




\section{1-Phenyl-1,2-dihydro-4H-benzo[d][1,3]oxazin-4-one (12)}

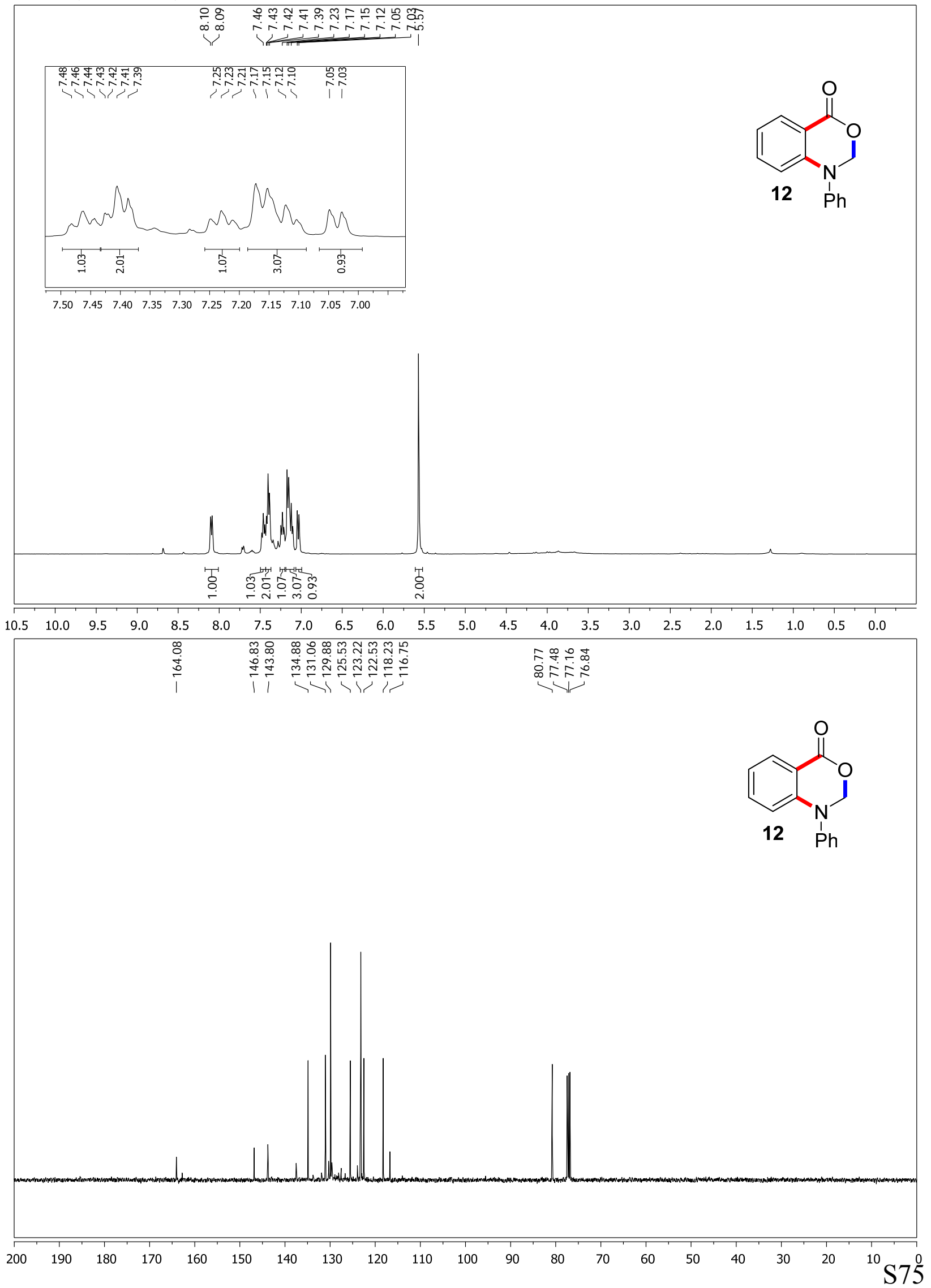

